\title{
Conceptualizations of remediation for practicing physicians
}

Citation for published version (APA):

Bourgeois-Law, G. A. (2020). Conceptualizations of remediation for practicing physicians. [Doctoral Thesis, Maastricht University]. Global Academic Press. https://doi.org/10.26481/dis.20200903gb

Document status and date:

Published: 01/01/2020

DOI:

10.26481/dis.20200903gb

Document Version:

Publisher's PDF, also known as Version of record

\section{Please check the document version of this publication:}

- A submitted manuscript is the version of the article upon submission and before peer-review. There can be important differences between the submitted version and the official published version of record.

People interested in the research are advised to contact the author for the final version of the publication, or visit the DOI to the publisher's website.

- The final author version and the galley proof are versions of the publication after peer review.

- The final published version features the final layout of the paper including the volume, issue and page numbers.

Link to publication

\footnotetext{
General rights rights.

- You may freely distribute the URL identifying the publication in the public portal. please follow below link for the End User Agreement:

www.umlib.nl/taverne-license

Take down policy

If you believe that this document breaches copyright please contact us at:

repository@maastrichtuniversity.nl

providing details and we will investigate your claim.
}

Copyright and moral rights for the publications made accessible in the public portal are retained by the authors and/or other copyright owners and it is a condition of accessing publications that users recognise and abide by the legal requirements associated with these

- Users may download and print one copy of any publication from the public portal for the purpose of private study or research.

- You may not further distribute the material or use it for any profit-making activity or commercial gain

If the publication is distributed under the terms of Article $25 \mathrm{fa}$ of the Dutch Copyright Act, indicated by the "Taverne" license above, 


\section{CONCEPTUALIZATIONS \\ OF REMEDIATION FOR \\ PRACTICING PHYSICIANS}

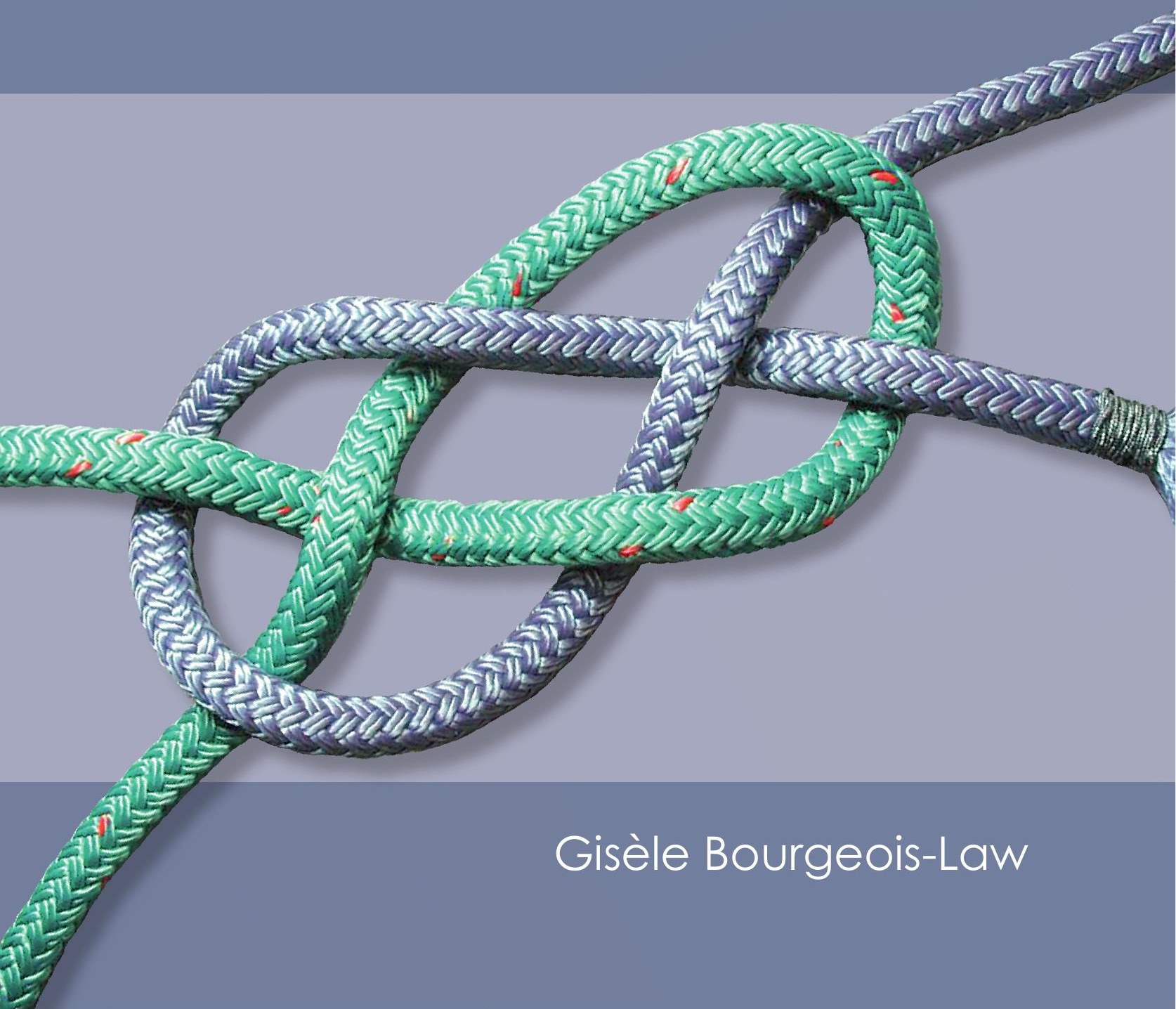



CONCEPTUALIZATIONS OF REMEDIATION FOR PRACTICING PHYSICIANS

by

Gisèle Angeline Bourgeois-Law 


\section{Maastricht University in Leanumin!}

within the School of Health Professions Education

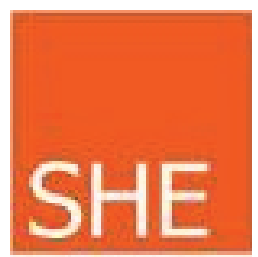

with funding for the review article 'Remediation of Practicing Physicians: Current and Alternative Conceptualizations' through a small grant from the Society of Directors of Research in Medical Education (a non-profit organization [501c3])

and funding for all empirical research through a substantial Medical Education Research Grant from the Royal College of Physicians and Surgeons of Canada.

Front cover illustration

The image (author unknown) was chosen as an analogy for remediation in practicing physicians. The two ropes of different colors are intertwined to form a strong knot, just as education and regulation are intertwined in the remediation of practicing physicians. Successful remediation programs require attention to both the educational and regulatory aspects of remediation.

(c) copyright G. Bourgeois-Law, Maastricht 2020

Printing: ProefschriftMaken | proefschriftmaken.nl

ISBN: 978-94-6380-902-3 


\title{
CONCEPTUALIZATIONS OF REMEDIATION FOR PRACTICING PHYSICIANS
}

\author{
Dissertation
}

to obtain the degree of Doctor at Maastricht University, on the authority of the Rector Magnificus, Prof. dr. Rianne M. Letschert in accordance with the decision of the Board of Deans,

to be defended in public on Thursday, 3 September, 2020 at 1600 hours

by

Gisèle Angeline Bourgeois-Law 


\section{Supervisors:}

Prof. Dr. Pim W Teunissen

Prof. Dr. Glenn Regehr, University of British Columbia, Vancouver

Prof. Dr. Lara Varpio, Uniformed University of the Health Sciences, Maryland

\section{Assessment Committee}

Prof. Dr. Eric W Driessen (Chair)

Prof. Dr. Fedde Scheele, Vrije Universiteit, Amsterdam

Dr. Renée Stalmeijer

Prof. Dr. Cees P.M. van der Vleuten

Dr. Jan-Willem Weenink, Erasmus University, Rotterdam 
Table of contents

$\begin{array}{lll}\text { CHAPTER } 1 & \text { Introduction }\end{array}$

CHAPTER 2 Remediation in Practicing Physicians: Current and Alternative Conceptualizations

CHAPTER 3 Education or Regulation? Exploring our Underlying Conceptualisations of Remediation for Practising Physicians

CHAPTER 4 Attitudes Towards Physicians Requiring Remediation: One-of-Us or Not-Like-Us?

CHAPTER 5 Educator, Judge, Public Defender: Conflicting roles for remediators of practicing physicians

CHAPTER 6 What's in a Name?

CHAPTER 7 Discussion

Summary

Samenvatting

Addendum: Valorisation paragraph

Acknowledgements

Curriculum Vitae 149

SHE Dissertation Series 

Introduction 



\section{Background}

The impetus for this PhD thesis arose out of my personal experience as the director of an assessment and remediation program for practicing physicians at the University of Manitoba from 1998-2008. The program had been set up a few years previously as a joint effort between the regulatory authority (the professional body that licenses physicians which, in Canada, are the provincial Colleges of Physicians and Surgeons) and the university with the aim of providing a 'one-stop shop' to which the Manitoba College could refer physicians who required a more detailed assessment of competence concerns. The program also aimed to offer a source of remediation preceptors that the College could trust. The program saw physicians with a variety of concerns including clinical competence issues, poor communication skills, and violations of sexual and other professional and personal boundaries. Surprisingly, clinical competence concerns proved to be the most challenging area for which to arrange remediation.

It would seem logical that competence concerns would be easier to remediate (and therefore to arrange remediation for) than a lack of professionalism or even ingrained poor communication habits. However, while we were able to develop a variety of subprograms to deal with specific challenges (e.g. a Professional Boundaries Program, a Communication Skills Program, and a Medical Record-Keeping Course, each of which provided intensive one-on-one support to physicians deemed to require practice change), arranging remediation for clinical competence issues remained a challenge in spite of all our efforts. Should co-existing mental health or addictions complicate any aspect of the remediation process, we could use the services of a well-organized provincial Physician Health Program. We even had access to a clinical psychologist who on occasion might diagnose cognitive or learning impairment. For purely competence concerns, however, we floundered. We could provide a detailed outline of the individual's gaps - what we called an 'educational prescription' - determined through a multi-day assessment that included a multiple-choice exam, a short-answer therapeutics exam, a structured oral interview to assess clinical reasoning, and an OSCE. And yet, if the remediatee's educational prescription involved more than reading or attending courses, we had little to offer. The program relied for many years on a single family physician who was willing to take peers into his practice for a remediatory period. It was virtually impossible to arrange remediation for specialists.

In 2008, I moved to a new university in a different province, a transition that raised my hopes for starting a similar program between British Columbia's provincial College and the university. I was aware that the College would welcome a remediation program; perhaps here, in a bigger province with four times the population, it would be easier to find physicians willing to remediate their peers. However, I encountered an unexpected obstacle: the Continuing Professional Development (CPD) Office worried a remediation program would brand them as 'an arm of the College'. This in turn might negatively affect their reputation among practicing physicians, which could in turn decrease regular CPD program attendance. This was a serious concern for an office that operates on a costrecovery basis. The program was never developed. 
With considerable frustration, I wondered: What was it about remediation, and remediation for clinical competence concerns in particular, that was so fraught? In my administrative mind-set, I thought that if we could only come up with the right structure, we would be able to engage university CPD offices and practicing physicians in the peer remediation of clinical competence. This frustration led me to consider pursuing a $\mathrm{PhD}$, with the vague goal of exploring and somehow discovering this 'right structure' that would facilitate stakeholder engagement. However, the process of developing the thesis proposal helped me realize that the problem might be more a conceptual than a process problem. More specifically, reviewing and discussing the literature suggested that we might need to look at remediation differently, rather than simply re-organize it.

\section{Literature on remediation in practice}

Until recently, very little was published on remediation in practice. This is not surprising if we consider that remediation has been the purview of regulators, whose ultimate aim is to protect the public. They are not researchers, which is to say that they are interested in sharing their experience with their peers, but not necessarily publishing in peerreviewed journals. To illustrate, a newsletter from the College of Physicians of Ontario dated 1999 published an abstract of a study comparing the performance of physicians who underwent remediation with an identified preceptor with that of a cohort who simply attended courses and read journals. $80 \%$ of the physicians assigned to preceptors improved on reassessment compared to $12.5 \%$ of those who attended courses and read journals; in fact, performance worsened for $75 \%$ of the participants in the second group. ${ }^{1}$ While this would seem very important information to share with the medical education community, a peer-reviewed paper reporting these data was never published. When it comes to remediation, much is shared in meetings that never makes it to a conference or publication, and documents often appear in the grey literature, only to disappear a few years later (in fact, some documents available at the beginning of my thesis research can no longer be retrieved).

Interestingly, even though assessment and remediation are inextricably linked (i.e. assessment precedes remediation, and sometimes also follows it thereby ensuring that remediation objectives have been met), we know a lot more about the assessment of practicing physicians than we do about remediation. Assessment programs, categorized into those aimed at assessing all physicians versus those aimed at providing a more detailed assessment of those whom have been identified in some manner as being potentially dyscompetent, have been widely studied..$^{2-4}$ These studies have looked at program description, evaluation of assessment tools, and in the case of potentially dyscompetent physicians, the risk factors for poor performance. ${ }^{5-24}$ It would certainly be possible to put together a 'best practices' paper on the assessment of practicing physicians based on the literature. However, while some of the program descriptions of more detailed assessment include mention of remediation, the remediation component is relatively minor. These assessment programs will develop remediation plans, but generally are not responsible for implementation of those same plans. The challenge lies with implementation - particularly for plans that go beyond knowledge acquisition (i.e. reading and course attendance) and 
require one-on-one supervision and mentoring. As one published review of assessment and remediation programs noted a decade ago: "This field of activity is characterized by the use of sophisticated methods for measuring performance/competence, but provision of remediation is more patchy and variable." ${ }^{\prime 7}$ Unfortunately, little has changed since then..$^{25}$ If we go back even further, in 1988, Dr. Dick Pierson, one of the founding members of the Coalition for Physician Enhancement and a tireless advocate for physician recredentialing and remediation, wrote that "Addressing the competence of all physicians assumes a willingness and a capability to address the support system that can stimulate physicians to remain competent, or in the case of failure, to provide a pathway to remediate deficiency. So much is clear. But there is much that is not yet clear: where does remedial education fit into the existing system? Who pays for it? Who provides it? Will leadership in designing the system be from the profession, the government, or from a third, external source?"4 More than 30 years later, we are still asking those same questions. Why is that? What do we know, or not know, about remediation that might help provide a clue as to why these questions remain largely unanswered in many jurisdictions?

We do know that the percentage of physicians deemed to require remediation varies from $0.5 \%$ to $30 \%$ depending on the jurisdiction and the criteria used to define remediation. ${ }^{26,27}$ We know that some physicians - those who are older, male, professionally (not necessarily geographically) isolated, or who trained outside their country of practice - are more likely to require remediation than others. 3,15,16,19,24,29-31 We also know that, without generalized peer-assessment programs aimed at reviewing every physician's practice, competence gaps may not be identified until they are quite severe. ${ }^{32-34}$ Finally, we know that shortterm success-rates, defined as meeting remediation learning objectives, hover around two thirds, and that younger physicians are more likely to successfully complete remediation. ${ }^{29,32,33,36-38}$

Perhaps other findings can offer additional clues as to why the development of remediation programs for clinical competence gaps in practicing physicians is such an elusive goal. Data on long-term outcomes is hard to come by. Ontario published a study of 81 physicians with significant practice concerns at initial assessment, who, when reassessed 6 years after intervention, as a group were practicing at least as well as their peers. ${ }^{38}$ Québec (unpublished data, International Physician Assessment Coalition 2014 meeting) presented results showing that $52 / 143$ physicians, or $36 \%$, whose remediation was considered successful i.e. they had achieved their learning objectives, had an unsatisfactory follow-up office assessment. We don't know why younger physicians are more likely to be successful in remediation; while increasing difficulty learning new habits and incorporating practice change may seem the obvious reason, one possibility put forward by a regulator is that older physicians closer to retirement may not have the same motivation as younger physicians. We also don't know best practices i.e. which programs are more likely to result in successful outcomes. A recent paper suggests that the most important research question in remediation would be what works in remediation, in what circumstances, and the underlying principles. ${ }^{39}$ While crucial, this wouldn't explain why we are still struggling with the same questions as 30 years ago. Most remediation-inpractice studies come from Canada or from the UK, although in the case of the latter, these are often hidden in the grey literature. The reasons for this variability between countries 
is unclear, but I would speculate are related to different political systems and cultural practices. For example, in several American states, physician licensing fees go directly into state general revenues and the state medical board (the regulator) has few funds to develop assessment and remediation programs (personal communication). In contrast, Canadian physician licensing fees go directly to the regulatory body, who is expected to play a significant role in ensuring quality of practice. More 'socialist' countries, such as Canada and the UK, are more likely to adopt assessment programs that can be viewed as limiting physician clinical autonomy than hyper-individualistic countries such as America; the more one assesses, the more opportunities will be found for remediation.

Perhaps looking for identified barriers would be useful. Remediation is expensive and poorly financed. It is complex, with most remediatees having deficits in more than one area. ${ }^{29,34,40-43}$ It can be difficult to find peers willing to remediate their colleagues; we might speculate that perhaps this is because another of Pierson's suggestions from 30+ years ago, that remediation requires a different type of medical educator with special expertise different from teaching learners, has not been widely acknowledged. ${ }^{36}$

Perhaps the problem is rooted in how the medical system is organized in North America. In the UK, the system funds remediation, thus removing an oft-cited barrier. ${ }^{44}$ However, a recent paper describing the UK experience outlines some of the frustrations and disappointments: "Despite a significant increase in the number of organizations reporting that they have official remediation policies in place, standardizing and professionalizing remediation has not been a smooth process... many of the problems identified in the late 90's still persist." 45 These problems include indemnity coverage, the role of the workplace, and fear of retribution when peers identify concerns. The workplace was identified as a contributing factor in $80 \%$ of cases, but the workplace/health authority is under no obligation to address these, and might even have an interest in focusing blame on the individual. Efforts to identify and address poor performance, without appropriate protections led to a "culture of blame, fear, and intimidation in the UK's health system." ${ }^{46}$

Thus, while the literature outlines some of the barriers and logistical issues, it also suggests that focusing on improving how we conduct remediation might not answer the question of why the medical profession struggles to address remediation of clinical competence. The combination of reading the literature and the personal experience mentioned earlier found me poised to begin my $\mathrm{PhD}$ research by examining the remediation processes for clinical competence gaps. However, when I described this research interest to colleagues, some of their comments gave me cause for pause. There was the comment from a colleague that she could "understand at a gut level" why physicians avoid involvement in the remediation of their peers but that she had difficulty in putting that reaction into words. Then there was the unexpectedly harsh comment from a regulator that "we only need to worry about remediation because we don't have enough doctors at the moment; if we did have enough doctors, they (doctors requiring remediation) would just lose their job."

The initial impetus for this thesis "how can we improve the remediation process for clinical competence gaps?" thus morphed into "why is remediation, particularly remediation of clinical competence gaps, so difficult to address at the practice level?" This led to related, 
foundational questions such as 'What is remediation?' If we're struggling to do 'it', do we need to rethink what 'it' is? An oft-quoted saying of Einstein's states:"IfI had an hour to solve a problem l'd spend 55 minutes thinking about the problem and 5 minutes thinking about solutions." The profession hasn't really thought about remediation beyond seeing it as a problem to be solved. The fact that we are still asking the same questions we were 30 years ago, led me to the realization that we need to re-think the problem we are attempting to address. This thesis aims to contribute to a better understanding of the problems we are trying to solve in the remediation of practicing physicians.

\section{Context}

Context plays a significant role in how remediation of clinical competence is organized. For example, in Canada, remediation is organized by the provincial colleges/regulatory bodies, who engage the provinces' universities to a greater or lesser extent in support of delivering remediation. The extent of that support varies widely depending on the province. The same appears to be the case in Australia and New Zealand (personal opinion from participation in remediation meetings in those countries). In the USA, programs may be organized by universities, or private not-for profits. ${ }^{3,20}$ As employees, UK physicians with clinical competence issues are dealt with in the same manner as other employees, i.e. with the same formal due process and safeguards. ${ }^{44}$ While most North American, and particularly US, doctors would cringe at the thought of being employees of the state, that situation does confer protection against arbitrary career-ending decisions by regulators and health authorities that self-employed physicians often lack. There is little in the literature from other countries, but as noted earlier, this does not mean that other jurisdictions don't carry out remediation; instead, it suggests that remediation has not been systematically studied and published or not published in English.

The Canadian context would appear to be a fruitful one in which to study remediation in practicing physicians. Canada has been a leader in this field; Hauer et al's review in 2009 found only 4 papers on remediation at the practice level, all of which were from Canada. ${ }^{47}$ The US has published widely on rehabilitation (i.e. programs for physicians with addictions or mental health issues), but not on remediation for clinical competence. One of the authors in Hauer's review, and the founder of the Physician Assessment and Clinical Education (PACE) Program at the University of California in San Diego, when speaking at a meeting of the International Physician Assessment Coalition in 2014 noted that "The United States spends over one trillion dollars annually on healthcare, yet has not produced a single study of practicing physician remediation" (Twitter \#IPAC2014). More recently, since we embarked on this thesis project, several papers have come out of the UK. $39,45,48,49$ However, a relatively recent literature review in BMJ Quality and safety noted that "seven of eight studies looking at outcomes for dyscompetence were from Canada".50

It is difficult to determine when formal remediation programs began in Canada. Regulatory authorities in Québec (1985) Ontario (1987) and Manitoba (1988) developed programs to conduct in-depth assessments of physicians with suspected competence deficiencies i.e. who in one way or another had come to the attention of the regulatory authority, generally 
as the result of a formal complaint by a patient, or more rarely, a fellow professional. Ontario and Manitoba chose to work through universities; the program in Manitoba is still in operation but the original PREP program in Ontario ceased some time ago. These in-depth assessments led to 'educational prescriptions', but since the primary purpose was assessment, the responsibility for actually ensuring that resources were available and that remediation programs were offered was unclear. ${ }^{5,33}$ Remediation often was, and in several cases still remains, a series of ad hoc arrangements. Québec's program remains the most advanced Canadian remediation program, a situation facilitated by factors not easily reproducible elsewhere. These include a) the licensing body is also the credentialing body, b) an early remediation program director benefited from close ties to a university family medicine program and was thus able to engage them in remediation, and c) francophone Québec physicians are less likely than their compatriots across Canada to move to other provinces so there is perhaps more willingness on the part of universities and individuals to remediate colleagues and graduates.

Two recent developments suggest that in the future more practicing physicians with focal or larger deficits in clinical competence will be identified. The first is the increasing number of Canadian provinces that have developed peer assessment programs that aim to screen the practice of all physicians at regular intervals. A similar process is happening in the UK with revalidation. ${ }^{51}$ The second is the introduction of Competency-Based Medical Education in Canada at the undergraduate and postgraduate levels and talk of extending the concept to the practice level. It is unclear how these physicians with identified gaps will be supported to bring their practice up to the required standard. The Canadian Physician Practice Improvement Initiative (PPI) is the latest effort at ensuring good practice nationwide. ${ }^{52}$ However, although that document includes a glossary of terms, remediation is conspicuously absent. The only mention of remediation is as a responsibility of medical schools/faculties of medicine, but not all medical schools/faculties of medicine are accepting that charge. Perhaps that is why the recent (2019) document, The Future of Medical Education in Canada - Continuing Professional Development (FMEC-CPD), which follows similar documents on undergraduate and postgraduate medical education, does not mention the word'remediation' at all. ${ }^{53}$ That is not to say that FMEC-CPD isn't a visionary document; it most assuredly is. It aims to transform CPD from physicians attending courses of their choosing, to individuals and teams using practice data to identify potential gaps and accessing resources to improve performance. It aims to address competence gaps before they become too wide - a laudable and even necessary objective. However, doing so will take time and resources, and in the meantime, the need for remediation is not going away.

While both the PPI and FMEC-CPD documents were published after the start of this PhD Project, I have included them in this introduction as they reinforce that not only are we still not answering Dr. Pierson's questions of "where does remedial education fit into the existing system? Who pays for it? Who provides it?, we don't appear to even want to address them. This further reinforces that we may need to think about remediation differently. However, rather than simply proposing new ways to conceptualize remediation, it might be useful to first understand the subtleties of how it is currently conceptualized, both in the literature, and by individuals in the field. 


\section{Thesis Aim:}

The aim of this thesis is to explore how the various stakeholder groups conceptualize the remediation of clinical competence in practicing physicians and how these various conceptualizations hinder or facilitate the process of addressing identified performance concerns related to knowledge and skills.

\section{Scope of thesis and definitions}

This thesis will focus on remediation for practicing physicians with gaps in clinical competence. As is the case in the postgraduate training (residency) realm, gaps in one area are frequently accompanied by gaps in another area, or by health, including mental health and addictions, issues. ${ }^{54-56}$ Some jurisdictions distinguish between remediation and rehabilitation, the latter being for health concerns; in this thesis, we focus solely on remediation of individuals without concomitant health problems. ${ }^{57}$

Practicing physician refers to a fully licensed physician who has completed all their undergraduate and postgraduate training.

Remediation refers to activities designed to bring a physician with clinical competence gaps back to the accepted standard of practice. It does not include rehabilitation as defined above.

\section{Reflexivity:}

I include a section on reflexivity since my past experience as the director of an assessment and remediation program for practicing physicians influenced my choice of dissertation topic and my decision to pursue a PhD. I came with experiences that provided me with insider knowledge (and helped me gain access to research participants), but also with fairly strong ideas about what was wrong with the system. As a member of both the Coalition for Physician Enhancement and the now defunct International Physician Assessment Coalition, I saw that colleagues managing remediation programs in North America, the UK, Australia and New Zealand had similar challenges to mine. While I did not personally carry out remediation, I conducted the intake interviews for the program and so was privy to remediatees' stories. The same characteristics that resulted in the need for remediation, such as poor decision-making, had often led to difficult circumstances in other aspects of their lives including in financial decisions, so that they were compelled to keep practicing despite having some insight into their deficiencies. Some were embarrassed and anxious to improve; others were defiant. In virtually all cases, it was impossible not to feel some degree of compassion for the remediatee and the situation in which they found themselves. Harold Shipmans (the UK GP who murdered over 200 of his patients) are fortunately extremely rare; individuals were struggling, not evil. ${ }^{58}$ 
I brought several assumptions and beliefs/attitudes towards remediation into my PhD research. First, I believe that attempts at remediation are always warranted, no matter the degree of the deficit. If there are no health or cognitive issues, it is impossible to predict who will and who won't do well, and to what degree. The individual might not be able to return to a full scope of practice, but some form of practice where they are able to use their limited skills to the service of the community is usually possible. I thus did not ask the question 'should we remediate' as part of the thesis, but did remain open to hearing participants express that view openly or indirectly.

Secondly, I contend that we need to focus on prevention at the earliest possible level: by addressing failure to fail at the training level; by teaching physicians how to manage the logistics of their practice so that they see that they can practice competently and still make a decent income and have time for their families; and by helping physicians learn how to retire. I persuaded several individuals to retire rather than undergo a competency assessment that would result in a humiliating loss or limitation of licensure. For some, money may have been an impetus to keep working, but more often, the motivation was linked to not knowing what to do with themselves when medicine had been their whole life. Since I had not worked in the field for several years, I was aware that in my interviews with participants, and in reading the transcripts, I had to look for ways in which remediation and remediatees might have changed (e.g. was the gender ratio different; were the external circumstances that led to remediation different, etc). I had to look to see if my presuppositions could be disconfirmed.

Finally, I brought to this research the firm belief that remediation is everyone's problem - the regulatory authority's, the university's, the health authority's and the professional (medical) association's. It had been my experience that it was difficult to engage individuals and organizations in remediation, and indeed, wanting to understand the reasons behind that unwillingness to engage with remediation was a strong impetus to do the $\mathrm{PhD}$. At the same time, I was aware that the situation was changing rapidly for reasons mentioned earlier, and that I had to be vigilant not to read more into what I heard than was there. Finally, it included the belief that remediation is a problem worth grappling with, for the sake of patients, the profession, and the individual dyscompetent physician.

\section{Thesis Summary}

\section{Chapter 2: What are the current explicit and implicit conceptualizations of the remediation of practicing physicians as evidenced in the literature?}

Chapter 2 consists of a scoping review of the literature on the remediation of practicing physicians, focusing on what is revealed explicitly or what might be revealed implicitly about the meaning of remediation for practicing physicians. The review includes the gray literature on remediation programs in North America and the United Kingdom, as well as other literature that contributes to our understanding of remediation, such as learning theories and theories on the sociology of medicine. 


\section{Chapter 3: How do remediation stakeholders conceptualize remediation?}

Various organizations have a stake in remediation. These stakeholders include regulatory authorities, certifying bodies, universities, and health authorities, among others. Chapter 3 explores how these different stakeholder groups grapple with the issue of remediation, and their underlying beliefs and assumptions regarding same. While Canada may be a favorable place in which to study stakeholder conceptualizations for reasons mentioned earlier, given that medical culture transcends national boundaries, findings would be applicable more widely. ${ }^{58}$

\section{Chapter 4: How do remediation stakeholder conceptualize individuals requiring remediation?}

Interactions with colleagues over the years suggested that, in spite of the common public perception that doctors 'stick together', physicians might not be as supportive of their struggling colleagues as they might be. Chapter 4 explores how the various stakeholder groups conceptualize individuals requiring remediation.

\section{Chapter 5: How do remediators conceptualize what they do?}

Remediators are those who are asked by regulatory and health authorities to oversee the learning and practice of physicians with significant competence gaps. This chapter explores the remediators' underlying conceptual frameworks around remediation for practicing physicians, their motivation for being involved in this activity, and how they view the physicians they are asked to remediate.

\section{Chapter 6: Commentary}

This commentary on a paper regarding the do's and don'ts of remediation, written at the end of the PhD and thus informed by our research findings, posits that if we don't change medicine's culture of independence and individual self-regulation, remediation will continue to be problematic no matter what we call it or how we organize it.

\section{Chapter 7: Discussion}

This final chapter discusses how the program of research has refined and shaped my conceptions of the nature of remediation for practicing physicians, and where those new conceptions might lead to. 


\section{REFERENCES}

1. Kaigas T, Ferguson B. Boosting Success at Remedial Education in Practicing Physicians Using Skilled Peer Preceptors. Ontario, Canada College of Physicians and Surgeons of Ontario 1999.

2. Donnon T, Al Ansari A, Al Alawi S, Violato C. The reliability, validity, and feasibility of multisource feedback physician assessment: a systematic review. Academic Medicine 2014;89 (3):511-516.

3. Norcross WA, Henzel T, Freeman KM, Milner-Mares JM, Hawkins RE. Toward Meeting the Challenge of Physician Competence Assessment: The University of California, San Diego Physician Assessment and Clinical Education (PACE) Program. Academic Medicine 2009;84 (8):1008-1014.

4. Pierson R. Competence, recredentialing, and remedial medical education. The Journal of Continuing Education in the Health Professions 1988;8 (4):321-325.

5. Page GG, Bates J, Dyer SM, et al. Physician-assessment and physician-enhancement programs in Canada. Canadian Medical Association Journal 1995;153 (12):1723-1728.

6. Finucane PM, Bourgeois-Law GA, Ineson SL, Kaigas TM. A Comparison of Performance Assessment Programs for Medical Practitioners in Canada, Australia, New Zealand, and the United Kingdom. Academic Medicine 2003;78 (8):837-843.

7. Humphrey C. Assessment and remediation for physicians with suspected performance problems: an international survey.The Journal of Continuing Education in the Health Professions 2010;30 (1):26-36.

8. Ferguson J, Wakeling J, Bowie P. Factors influencing the effectiveness of multisource feedback in improving the professional practice of medical doctors: a systematic review. BMC Medical Education 2014;14 76.

9. Lockyer J. Multisource feedback: can it meet criteria for good assessment? The Journal of Continuing Education in the Health Professions 2013;33 (2):89-98.

10. Wenghofer EF, Way D, Moxam RS, Wu H, Faulkner D, Klass DJ. Effectiveness of an enhanced peer assessment program: Introducing education into regulatory assessment. The Journal of Continuing Education in the Health Professions 2006;26 (3):199-208.

11. Goulet F, Jacques A, Gagnon R, Charlin B, Shabah A. Poorly performing physicians: does the Script Concordance Test detect bad clinical reasoning? The Journal of Continuing Education in the Health Professions 2010;30 (3):161-166.

12. Chesluk BJ, Reddy S, Hess B, Bernabeo E, Lynn L, Holmboe E. Assessing interprofessional teamwork: pilot test of a new assessment module for practicing physicians. The Journal of Continuing Education in the Health Professions 2015;35 (1):3-10.

13. Gagnon R, Jacques A, Billard M, Goulet F. Determining the number of patient charts necessary for a reliable assessment of practicing family physicians' performance. Evaluation \& the Health Professions 2010;33 (1):109-122.

14. Yanes AF, McElroy LM, Abecassis ZA, Holl J, Woods D, Ladner DP. Observation for assessment of clinician performance: a narrative review. BMJ Quality \& Safety 2016;25 (1):46-55.

15. Alam A, Klemensberg J, Griesman J, Bell CM. The characteristics of physicians disciplined by professional colleges in Canada. Open Medicine 2011;5 (4):e166-172.

16. Bismark MM, Spittal MJ, Gurrin LC, Ward M, Studdert DM. Identification of doctors at risk of recurrent complaints: a national study of healthcare complaints in Australia. BMJ Quality \& Safety 2013;22 (7):532-540. 
17. Eva KW. Editorial - Dangerous Personalities. Advances in Health Sciences Education 2005;10 (4):275-277.

18. Garfinkel PE, Bagby RM, Waring E, Dorian B. Boundary Violations and Personality Traits among Psychiatrists. The Canadian Journal of Psychiatry 1997;42 (7):758-763.

19. Kohatsu ND, Gould D, Ross LK, Fox PJ. Characteristics associated with physician discipline: a case-control study. Archives of Internal Medicine 2004;164 (6):653-658.

20. Korinek L, Thompson L, McRae C, Korinek E. Do Physicians Referred for Competency Evaluations Have Underlying Cognitive Problems? Academic Medicine 2009;84 (8):1015-1021.

21. Mengel MB. Physician ineffectiveness due to family-of-origin issues. Family Systems Medicine 1987;5 (2):176-190.

22. Roback HB, Strassberg D, lannelli RJ, Finlayson AJR, Blanco M, Neufeld R. Problematic physicians: a comparison of personality profiles by offence type. Canadian Journal of Psychiatry 2007;52 (5):315-322.

23. Studdert DM, Bismark MM, Mello MM, Singh $\mathrm{H}$, Spittal MJ. Prevalence and Characteristics of Physicians Prone to Malpractice Claims. New England Journal of Medicine 2016;374 (4):354-362.

24. Unwin E, Woolf K, Wadlow C, Dacre J. Disciplined doctors: does the sex of a doctor matter? A cross-sectional study examining the association between a doctor's sex and receiving sanctions against their medical registration. BMJ Open 2014;4 (8):e005405.

25. Goulet F, Thiffault J, Ladouceur R. Remediation and rehabilitation programmes for health professionals: challenges for the future. BMJ Quality \& Safety 2017;26 (12):941-943.

26. National Clinical Assessment Service. NCAS Casework The First Eight Years. 2009

27. Personal Comunication, College of Physicians and Surgeons of British Columbia (2105 and 2019).

28. Norton PG, Ginsburg LS, Dunn E, Beckett R, Faulkner D. Educational interventions to improve practice of nonspecialty physicians who are identified in need by peer review. The Journal of Continuing Education in the Health Professions 2004;24 (4):244-252.

29. Goulet F, Jacques A, Gagnon R. An innovative approach to remedial continuing medical education. 1992-2002. Academic Medicine 2005;80 (6):533-540.

30. Grace ES, Wenghofer EF, Korinek EJ. Predictors of physician performance on competence assessment: Findings from CPEP, the Center for Personalized Education for Physicians Academic Medicine 2014;89 (6):912-919.

31. Alam A, Matelski JJ, Goldberg HR, Liu JJ, Klemensberg J, Bell CM. The characteristics of international medical graduates who have been disciplined by professional regulatory colleges in Canada: a retrospective cohort study. Academic Medicine 2017;92 (2):244-249.

32. Hanna E, Premi J, Turnbull J. Results of Remedial Continuing Medical Education in Dyscompetent Physicians. Academic Medicine 2000;75 (2):174-176.

33. Goulet et al Conference Presentation 2014 Joint Conference of IPAC and CPE; Druids Glenn Resort, Ireland

34. Smith R. Remediation Working Group Report. London: Academy of Medical Royal Colleges 2012.

35. Leape LL, Fromson JA. Problem doctors: is there a system-level solution? Annals of internal medicine 2006;144 (2):107-115.

36. Lillis S, Takai N, Francis S. Long-Term Outcomes of a Remedial Education Program for Doctors With Clinical Performance Deficits. The Journal of Continuing Education in the Health Professions 2014;34 (2):96-101. 
37. Rhydderch $M$, Matthews $P$, Beech $M$. The advanced training practice network: providing prescribed further training for general practitioners in Wales. Education for Primary Care 2007;18 (5):572-581.

38. Norton PG, Dunn EV, Beckett R, Faulkner D. Long-term follow-up in the Peer Assessment Program for nonspecialist physicians in Ontario, Canada. The Joint Commission Journal on Quality Improvement 1998;24 (6):334-341.

39. Price T, Brennan N, Cleland J, et al. Remediating doctors' performance to restore patient safety: a realist review protocol. BMJ open 2018;8 (10):e025943.

40. Whiteman J, Jamieson A. Remediation with Trust, Assurance and Safety. Education for Primary Care 2007;18 (6):665-673.

42. Cohen D, Rhydderch M. Making an objective assessment of a colleague's performance. The Clinical Teacher 2010;7 (3):171-174.

42. Klass D. Assessing Doctors at Work - Progress and Challenges. New England Journal of Medicine 2007;356 (4):414-415.

43. Cerda JJ, Van Susteren TJ, Hatch R, Herkov M. Remedial education: can this doctor be saved? Transactions of the American Clinical and Climatological Association 2000;111:188-195; discussion 196-197.

44. SommersetTrust. Remediation, Re-skilling and Rehabilitation Policy for Medical Staff. UK: National Clinical Assessment Service; May 2012.

45. Price T, Archer J. UK Policy on Doctor Remediation: Trajectories and Challenges. The Journal of Continuing Education in the Health Professiosn 2017;37 (3):207-211.

46. The UK general election: a manifesto for health. Editorial The Lancet 2015;385 (9971):829.

47. Hauer K, Ciccone A, Henzel T, al e. Remediation of the Deficiencies of Physicians Across the Continuum from Medical School to Practice: A thematic Review of the Literature. Academic Medicine 2009;84 (12):1822-1832.

48. Bourne T, De Cock B, Wynants L, et al. Doctors' perception of support and the processes involved in complaints investigations and how these relate to welfare and defensive practice: a crosssectional survey of the UK physicians. BMJ Open 2017;7 (11):e017856.

49. Prescott-Clements L. Rethinking Remediation: A Model to Support the Detailed Diagnosis of Clinicians' Performance Problems and the Development of Effective Remediation Plans. The Journal of Continuing Education in the Health Professions 2017;37 (4):245-254.

50. Weenink J-W. Getting back on track: a systematic review of the outcomes of remediation and rehabilitation programmes for healthcare professionals with performance concerns. $B M J$ Quality \& Safety 2017;26 (12):1004-1014.

51. Archer J, de Bere SR. The United Kingdom's experience with and future plans for revalidation. The Journal of Continuing Education in the Health Professions 2013;33 Suppl 1:S48-53.

52. Federation of Medical Regulatory Authorities of Canada. Physician Practice Improvement. Ottawa, Canada 2016. https://fmrac.ca/physician-practice-improvement/ (Accessed July 5, 2016)

53. Association of Faculties of Medicine of Canada. Future of Medical Education in Canada-Continuing Professional Development. Ottawa, Canada 2019. https://www.fmec-cpd.ca/ (Accessed May 10, 2019)

54. Dupras DM, Edson RS, Halvorsen AJ, Hopkins Jr RH, McDonald FS. "Problem Residents": Prevalence, Problems and Remediation in the Era of Core Competencies. The American Journal of Medicine 2012;125 (4):421-425. 
55. Guerrasio JMD, Brooks EP, Rumack CMMD, Christensen AJDMS, Aagaard EMMD. Association of Characteristics, Deficits, and Outcomes of Residents Placed on Probation at One Institution, 2002-2012. Academic Medicine 2016;91 (3):382-387.

56. Guerrasio J, Garrity MJ, Aagaard EM. Learner deficits and academic outcomes of medical students, residents, fellows, and attending physicians referred to a remediation program, 20062012. Academic Medicine 2014;89 (2):352-358.

57. The Back on Track Framework for Further Training. UK: National Clinical Assessment Service; 2010. (Accessed November 15, 2015)

58. Jackson T, Smith R. Harold Shipman. BMJ : British Medical Journal 2004;328 (7433):231-231.

59. Taylor JS. Confronting "culture" in medicine's "culture of no culture". Academic Medicine 2003;78 (6):555-559. 



\section{Remediation in Practicing Physicians: Current and Alternative Conceptualizations}

Gisèle Bourgeois-Law | Pim W. Teunissen | Glenn Regehr 


\section{Abstract}

Suboptimal performance in practicing physicians is a decades-old problem. The lack of a universally accepted definition of remediation, the paucity of research on best remediation practices, and the ongoing controversy regarding the institutional responsibility for enacting and overseeing this activity suggests that the remediation of physicians is not merely a difficult problem to solve, but a problem that the community does not grapple with meaningfully. Undoubtedly, logistical and political considerations contribute to this state of affairs; however, other underlying conceptual issues may also play a role in the medical profession's difficulties in engaging with the challenges around remediation.

Through a review of the medical education and other literatures, the authors examined current conceptualizations of both remediation itself and the individual being remediated, as well as how the culture of medicine influences these conceptions. The authors explored how conceptualizations of remediation and the surrounding culture might affect not only the medical community's ability to support, but also its willingness to engage with physicians in need of remediation.

Viewing remediation as a means of supporting practice change - rather than as a means of redressing gaps in knowledge and skill - might be a useful alternative conceptualization, providing a good place to start exploring new avenues of research. However, moving forward will require more than simply a reconceptualization of remediation; it will also necessitate a change in how the community views its struggling members and a change in the medical culture that currently positions professional autonomy as the foundational premise for individual practice improvement. 
Suboptimal performance by practicing physicians possesses all the characteristics of a "wicked problem," in that constantly evolving social and political factors, which vary across time and jurisdiction, render a definitive solution highly unlikely. ${ }^{1}$ Perhaps not surprisingly therefore, the most commonly proposed solution to this problem, individual remediation, is itself a "wicked problem" - one that the medical profession has struggled with for decades. Problems with remediation manifest in several forms: conceptually, no coherent (much less universally accepted) definition of remediation is available in the medical community; academically, research on best remediation practices is lacking; and administratively, institutional responsibility for enacting and overseeing remediation engenders controversy. Each of these manifestations suggests that the remediation of practicing physicians is not merely a difficult problem to solve, but a problem that the community struggles to grapple with meaningfully. Based on the premise that the first step in addressing a problem is defining its characteristics, we conducted a literature review to determine the state of the art regarding remediation in practicing physicians.

\section{The Scoping Review}

\section{Method}

We carried out a scoping review between July 2016 and January 2017 with the goals of determining the breadth of the literature on remediation, identifying gaps, and exploring current and potential conceptualizations. ${ }^{2}$ We focused specifically on the remediation of practicing physicians struggling with clinical competence issues. We did not include articles about physicians whose health concerns (e.g. mental illness, addictions) might affect competence. We therefore excluded articles that referenced remediation only during training, those that focused solely on assessment, and those that focused exclusively on remediation for issues related to professionalism, rehabilitation, or re-entry into practice after a prolonged absence for reasons unrelated to clinical competence. We included concept papers and editorials. We included any articles published through December 2016. We searched PubMed, Eric, Web of Science, and Google Scholar, using the following terms: remediation practicing physicians, remedial continuing medical education, dyscompetence practicing physicians, competence practicing physicians, and revalidation. We also scanned the reference lists of the articles we identified, as well as those of two relevant books. ${ }^{3,4}$ Finally, because much of the available information on remediation at the practice level is located on government and organizational websites, we also searched (using the search terms above) the gray literature using the search engine DuckDuckGo, as well as the Twitter feeds and websites of relevant organizations (e.g. the Coalition for Physician Enhancement, a consortium of organizations with an interest in physician performance improvement). We reviewed the abstracts of identified articles to determine their relevance. Then we extracted information from those selected articles and documents pertaining to remediation in practicing physicians.

\section{Results}

Using the above criteria, we identified 33 papers and documents from the grey literature (Appendix A). The results of the literature search confirmed our belief that remediation is a "wicked" problem. First, we noticed the relative absence of research - educational 
or otherwise - published on the remediation of practicing physicians. Since 2009 when Hauer and colleagues commented explicitly on the lack of research on the remediation of practicing physicians, we could find only two new studies. ${ }^{5-7}$ In contrast, during that same time, several studies addressed the assessment of competence in practicing physicians, suggesting that addressing physicians in need of remediation is more difficult than determining physicians' competence in the first place. ${ }^{8-17}$

Second, we determined that definitions of remediation are surprisingly scarce. For example, Kalet and Chou's seminal book Remediation in Medical Education begins with a chapter on defining competence, not remediation - and the main delineation of the concept of remediation is found in the book's sub-title, "A mid-course correction," not in the text. ${ }^{4}$ Similarly, published articles rarely define remediation per se; instead, definitions are implied in the descriptions of underlying causes for its need (e.g. mental health issues), or the descriptions of groups more likely to require it (e.g. elderly physicians). The general lack of clarity (or specificity) about what falls under the umbrella of remediation is not simply a matter of semantics: this ambiguity undermines the community's ability to determine the incidence of remediation, thus creating difficulties both for resource planning and for comparing success rates across programs and jurisdictions. ${ }^{18,19}$

Perhaps the most telling indicator of the wickedness of the problem of physician remediation lies in the apparent hesitation by institutional stakeholders to become engaged in the remediation process. Unlike the assessment and identification of physicians in difficulty, which regulatory and health authorities view as part of their mandate, no single stakeholder group agrees that remediation falls under its mandate. Depending on the jurisdiction, regulatory authorities, universities, health authorities, and specialty boards may have a greater or lesser role, or in some cases, no role at all. ${ }^{20-23}$ This lack of clear authority frequently leads (with a few exceptions) to ad hoc, rather than programmatic, solutions for the remediation of physicians with clinical deficiencies. 8,24,25 While political issues undoubtedly play a role in this state of affairs, this absence of clear responsibility may be more indicative of underlying issues with how the medical community conceptualizes remediation.

\section{Conceptual Synthesis}

Based on these findings, we concluded that to untangle the wicked problem of remediation, addressing the conceptual ambiguity that surrounds it is vital. To this end, we conducted a second, conceptual review of remediation. We explored various literatures that we felt might inform potential conceptualizations of remediation including literatures on learning, on change, on the influence of personality on behavior, and on the sociology of medicine. To identify these literatures, we started with books or articles on specific topics including Kalet and Chou's book on remediation and Bleakley's book, Medical Education for the Future: Identity, Power and Location. ${ }^{4,26}$ Next, we followed references of articles discovered through our earlier search, searched our files looking for potentially relevant articles, and spoke with colleagues who suggested other possibilities. The identification and exploration of these new domains of literature was more organic than systematic, emerging from discussions with a broad range of colleagues. The goal was not to be 
exhaustive, but rather to develop a meaningful synthesis of these literatures to inform potential conceptualizations of remediation.

For the purposes of this conceptual analysis, we have focused on what we see as the "iconic" physician in need of remediation, the physician for whom there are identified concerns related to knowledge, skills, and performance. When appropriate, we contrast this physician with physicians who require particular assistance due to health problems including physical, mental/emotional, and/or addiction issues. We note that while several issues may be at play (either sequentially or simultaneously), we suggest - and, in fact, argue - that, conceptually, it is the physician who is perceived as struggling in practice, but who has no "medical" reason (e.g. underlying addiction or mental health problem) that most challenges the medical community. This is the physician with which the medical profession must come to terms.

\section{Findings and Discussion}

The synthesis of this broader set of literatures generated three conceptual domains that may be contributing to the medical community's difficulties in engaging with the challenges around remediation: (1) the conceptualization of remediation itself; (2) the conceptualization of the person being remediated; and (3) the culture of medicine as an influence on these conceptions. While these three categories are inextricably intertwined, we discuss each separately in the interests of clarity.

\section{Conceptualizations of remediation}

The first domain identified was the ambiguity of the underlying conception of remediation, including the goals of remediation and the processes invoked to achieve those goals. Remediation in practicing physicians is most frequently described as an educational process. $^{7,19,27}$ The literature suggests that it is usually conceptualized as a means of addressing a gap in an individual's knowledge or skills (including knowledge and skills in the areas of ethics and professionalism)..$^{28}$ We posit, however, that conceptualizing remediation in this fashion can lead the community of physicians down some potentially thorny paths.

First, conceptualizing remediation as simply addressing a gap in knowledge or skill can lead to an over-focus on knowledge acquisition as the solution. While knowledge is important (indeed, practitioners may need to acquire or re-learn knowledge or skills), strong evidence in the continuing medical education (CME) literature suggests that knowledge alone is insufficient to accomplish practice change. ${ }^{29,30}$ Individual performance is influenced, for better or worse, by the attributes and skills of colleagues, by system resources (e.g. the availability of specialized equipment and trained personnel), and by organizational culture - the myriad sociocultural and systemic factors that we term the "social determinants of performance."31 Remediation as a purely educational exercise that does not address the contextual factors supporting or hindering competence in individuals is, in and of itself, unlikely to be sufficient to address significant performance gaps. 
Second, the model of remediation as (more) education fails to take into account a number of sociopolitical and economic issues associated with being in the learner role. That is, the remediation of practicing physicians seems to be conceptualized similarly to the remediation of residents: similar attributes are thought to be required of preceptors in both cases, (although these are not generally specified), and similar methods are promoted (e.g. directed reading, required courses, and possibly further clinical rotations). ${ }^{32,33}$ Importantly, however, the sociopolitical and economic considerations at play in practice differ from those in residency: residents are considered learners, and mistakes are viewed as opportunities to learn. ${ }^{34}$ Additionally, residents are not ultimately responsible for the patients they treat, so they do not suffer the same kind of loss of authority that "supervised" practitioners potentially experience..$^{35}$ Also, resident remediation is not overlaid with economic considerations, such as funding for the preceptor and physician in remediation. Thus, treating the remediation of physicians as simply an educational problem risks omitting from the equation significant factors that will affect its practices and its outcomes.

If remediation is more complex than simply filling an educational gap, how might the medical community better conceptualize it? In psychology textbooks learning is often defined as a "relatively permanent change in behavior brought about by experience or practice."36(p176) In this spirit, perhaps remediation might better be conceptualized as supported practice change. Conceptualizing remediation as practice change allows the profession to recognize that remediation is more of a change management problem than an educational issue. The CME literature notes the difficulties that even highly effective practicing physicians encounter in changing their practice to conform to new developments. ${ }^{37-39}$ As a result, CME programs are evolving to focus on practice change with the understanding that such change requires resources, including practice tools and mentorship. ${ }^{40-42}$ In this sense, reconceptualizing remediation as a change management process would be congruent with viewing remediation as situated on the continuum of CME. Physicians requiring remediation would sit on one extreme of the continuum since, compared to colleagues, they simply have more significant change to implement and/or more challenging barriers, including grappling with the emotional toll of being identified as needing remediation and its attendant effect on self-esteem (see below).

Successful remediation, therefore, will likely require, at a minimum, the same magnitude of resources as other practice change initiatives. A benefit of conceptualizing remediation as supported practice change might enable physicians in remediation and their preceptors to better identify underlying factors that could hinder or facilitate that change and, in turn, tailor remediation to the individual to achieve better long-term outcomes. The medical community's ability and willingness to view and enact remediation as a change management process - as simply an extension of the change management process increasingly promoted in the CME literature - has the potential not only to increase the effectiveness of remediation but also to destigmatize it. One barrier, however, to considering remediation as part of continuing professional development might be how the profession conceptualizes the clinicians requiring remediation. 


\section{Conceptualizations of the individual requiring remediation}

Perceptions of struggling colleagues are shaped by concepts of competence and the causes of dyscompetence. As Hodges has noted, competence is a "sociohistorical construction with links to power."43(p.40) That is, those with power possess significant influence in defining what constitutes competence or the lack thereof. The same might be said for deciding which individuals and which activities require remediation. How the medical community views competence/dyscompetence may well have important implications for how it frames and approaches those physicians requiring remediation (and, in turn, how it frames and approaches the process of remediation).

Many investigators, seeking to understand risk factors for underperformance and potential predictors of successful remediation, have sought to determine demographic and personal characteristics of physicians requiring remediation. ${ }^{10,44-46}$ Such studies may inadvertently reinforce a perception that the physicians requiring remediation possess predisposing personal characteristics that have somehow led to their present situation and may further lead to the belief that remediation outcomes depend almost exclusively on the individual being remediated. ${ }^{31}$ Thus, a focus on the stable personal characteristics of physicians requiring remediation such as age, gender, country of training/origin, or ethnicity may contribute to the reluctance to address dyscompetence: these personal characteristics will make behavioral and practice changes difficult. If the cause is a personal characteristic, then no remediation (of any form) will be sufficient to effect change in the physician requiring it. Perhaps more importantly, a focus on personal characteristics could also lead clinicians to view a colleague in remediation as somehow different from themselves, which only compounds that individual's struggles. Such a view allows clinician coworkers to distance themselves or exclude the struggling physician from the community of practice - rather than embrace a colleague who is in particular need of support..$^{10,47}$

This process of "othering" is well described in the psychology literature, which elaborates on several perceptual biases that could influence the conceptualization of individuals in need of remediation. For example, attribution theory describes the human tendency to see other people's behavior as reflective of their personality or characteristics, and our own behavior as resulting from external circumstances. ${ }^{48}$ The "just world phenomenon" explains the human need to believe that we all live in a world where people ultimately get what they deserve. ${ }^{49}$ This bias often results in blaming the victim, or in this case, perhaps a lack of empathy for the situation in which dyscompetent practitioners find themselves. Similarly, humans tend to judge actions based on outcomes whether or not the latter were under the control of the people involved. ${ }^{50}$ These biases frequently result in a failure to distinguish between the behavior and the person. Trainees and practitioners alike, for example, may be told that they themselves are unprofessional rather than that their behavior at a point in time was unprofessional. Similarly, people are labelled "incompetent" in spite of overwhelming evidence that competence is both content and context specific. ${ }^{51-53}$ 
One manifestation of the process of othering is the difference in systemic responses to physicians in need of rehabilitation versus those in need of "reskilling" (i.e. medical treatment versus educational training). All Canadian provinces and almost all U.S. states boast a physician health program (PHP) to support addicted, stressed, and even in some cases, disruptive physicians. Yet relatively few formal programs exist for those requiring remediation to address deficits in knowledge, skills, or clinical reasoning. One reason for this discrepancy might be how the community implicitly interprets the source of these problems. Physicians are trained to believe that most illnesses are not any individual's fault, so they attach less stigma to an individual patient. By contrast, physicians may see dyscompetence as squarely the fault of the practitioner needing remediation. The ill physician invokes others' support, but a physician who has allowed him or herself to become dyscompetent may not. This lack of support or empathy, this blame, may be, as we suggested in the introduction, the reason that it is the physician perceived as struggling in practice, but with no medical diagnosis, who constitutes the challenge with which the medical community must come to terms.

We emphasize that focusing on demographics or the personal characteristics of physicians requiring remediation risks not only underestimating the influence of context on competence and remediation, but also succumbing to stereotyping (such as ageism) in ways that isolate rather than embrace and support the physician in trouble - to the detriment of both the individual practitioner and the broader community. ${ }^{54}$ Each physician requiring remediation is a unique individual practicing in a context that is never exactly the same as that of his or her peers. We thus return to our concept of remediation as supported practice change and the need to consider sociocultural and systemic factors in addition to those that are unique to the specific individual and relevant to the situation. One characteristic that virtually all physicians share, however, is their embeddedness in the culture of medicine.

\section{The effect of medicine's culture on conceptions of remediation}

The culture of medicine, in which physicians both train and practice, appears surprisingly homogeneous across different countries and health care systems. This culture includes the practice of "shaming and blaming" a physician for errors, dictates the types of errors physicians will tolerate in trainees or colleagues, and inculcates the reluctance to challenge a colleague's practice. ${ }^{34,55-57}$ Each of these phenomena likely has some bearing both on the willingness to remediate and on the determination of who and what is considered remediable. Perhaps the cultural concept that has the greatest bearing on the willingness to remediate, however, is that of professional autonomy.

Friedson has argued that "the single zone of activity in which autonomy must exist in order for professional status to exist is in the content of work itself." ${ }^{\prime \prime(p . ~ 255)}$ This concept of clinical autonomy acknowledges that the judicious use of knowledge and skills, rather than the blind following of standards, is not only more intellectually satisfying for the professional but usually in everyone's best interest, including that of patients. While the concept of the autonomous physician is evolving and the need for some form of oversight 
is increasingly accepted, clinical autonomy remains a core defining characteristic of the profession. Autonomous practice is a right and a privilege that residents work hard to merit, and the acquisition of which marks a rite of passage at the end of training. Removal of that autonomy occurs only in cases of dangerous incompetence or negligence.

A less frequently discussed component of professional autonomy is that of educational autonomy, the freedom of the individual physician to decide what and how to learn. Educational autonomy is also evolving - in that a certain number of CME hours and even types of CME are now mandatory in many jurisdictions. However, the core underlying concept of individual freedom to manage one's own ongoing professional development remains unchallenged, with one exception. As with clinical autonomy, removal of educational autonomy is invoked when physicians have been shown to be negligent (i.e. not to have managed their learning so as to keep up to date in their knowledge and/or skills).

Remediation can thus be conceptualized as involving the removal of an individual physician's professional right and personal responsibility to self-determine a satisfactory level of competence and to self-regulate when, how, and what to learn. Unlike mandatory continuing professional development, which merely stipulates that physicians must continue to learn (and offer evidence of having participated in the learning activity), remediation prescribes what must be learned and determines when satisfactory learning has occurred. The individual physician has failed to self-regulate, so another authority is assuming that responsibility. In the process, the physician in remediation might be said to have lost a core defining characteristic of the profession: autonomy and the associated right to self-regulate. ${ }^{58}$ This loss may, in turn, lead to the othering (described earlier). Colleagues may feel not only discomfort for and around a physician in remediation; but if disgrace has been public, they may also feel, at least to some extent, resentment for giving the practice, institution, or even the profession a bad name. No wonder, then, that remediation is fraught with personal, cultural, and political baggage.

Interestingly, the removal of autonomy is not always accompanied by a complete removal of responsibility for addressing the deficit. For example, some remediation programs require physicians to identify the resources required for remediation and to pay the entire cost themselves. The unexpressed reason underlying this financial liability may be that since the situation is the individual physician's "fault," it is also his/her sole responsibility to find ways to remediate it. Now is perhaps the time for the profession to consider a more social model of remediation. Increasingly, the medical community is recognizing that competence is a team sport. ${ }^{31}$ Despite this nascent awareness, the culture of medicine in which each person is responsible for one's own competence and only for one's own competence might require challenging. While individual physicians should not be deemed responsible for their colleagues' competence, we argue that they are responsible to their colleagues and to the profession. Together, physicians are collectively responsible to contribute to the culture and system that motivates the ongoing performance improvement of everyone. We believe the same model should apply to remediation: that everyone is responsible to dyscompetent physicians to support their successful return to competence. Of course, that responsibility will vary depending on individual roles and the 
circumstances, but at a minimum, responsibility entails accepting or continuing to include a physician in remediation within the practice or community. This shared responsibility is perhaps not so much a culture change, as it is a re-interpretation of the requirements inherent in the concepts of professional autonomy and self-regulation.

\section{Implications}

Our analysis has led us to conclude that currently the medical community views and experiences remediation, ultimately, as a "de-professionalization" because it removes from the individual two important characteristics of a professional: clinical autonomy and self-regulation. This de-professionalization makes remediation emotionally and politically fraught for not only individuals but for the profession as a whole. Understanding remediation as a loss of autonomy and therefore a form of deprofessionalization might enable the medical community to understand why grappling with this wicked problem has proven so difficult for so long and in so many quarters.

As a step toward redressing the fraught nature of remediation, we suggest characterizing remediation as supported practice change that focuses on the social and cultural aspects of collective competence, rather than on the individual personalities of those requiring remediation. We believe that explicitly taking into account the influence of medical culture regarding remediation might lead to better outcomes. We stress that we view the concept of supported practice change not as a solution to the problem of remediation, but as a different way of framing the problem. That is, reconceptualizing remediation as supported practice change suggests new lines of exploration that the community might profitably pursue.

For example, remediation as supported practice change aligns with social learning theories. Until recently, individualistic learning theories such as adult learning theory, experiential learning, and reflective learning/practice, have had the most traction in medical education, particularly in CME. ${ }^{26,59-62}$ However, individualistic learning theories are not always sufficient either to diagnose why a particular physician is struggling or to develop a successful remediation plan. Such theories do not adequately take into account the considerable sums of money, time, and effort that are often required to enable competent practitioners to alter even one small component of their practice patterns. ${ }^{36,63}$ Acknowledging - as social learning theories do - that it is impossible to separate individual knowledge, motivation, affect, and ability from the influence of context and culture ${ }^{59}$ would not diminish individual accountability and/or the responsibility of each physician to remain up to date and to provide competent care. Acknowledging the interdependence of individual and contextual factors (the social determinants of performance) would, however, lead to remediation strategies that take into account the learning tools available to a struggling physician, the processes required for practice change (see below), the division of labor, and the community in which the physician practices. ${ }^{64}$ Starting with social learning theories might stimulate questions such as, "How might we effectively use a physician's community of practice in remediation?" or "How might we determine the influence of context in a particular case of clinical dyscompetence?" Paradoxically, 
remediation based on social rather than individual learning theories might entail a plan that is tailored to a specific individual in a particular situation; in fact, it would be the polar opposite of a communal one-size-fits-all approach.

Conceptualizing remediation as supported practice change would also lead to the literature on change management. That literature explores issues such as the motivation and ability to change, and the facilitators and inhibitors of change (in this case, the facilitators and inhibitors of remediation). ${ }^{65}$ Understanding remediation as supported practice change would inspire questions such as, "How do conceptualizations of remediation and of struggling physicians interact with medical culture to shape whether/ how various institutional stakeholders and leaders envisage addressing remediation?"; "What structures can we create to ensure long-term practice change rather than shortterm achievement of learning objectives?"; or even "How do we determine to what extent needed practice change will be possible for a particular physician in a given context?" Models such as the Influencer model (which is based on the work of Albert Bandura) or the theory of planned behavior, which is used to understand the effect of public health interventions, might be explored and adapted to planning and studying remediation. ${ }^{65}$

Physicians requiring remediation have deviated from professional and cultural norms. The medical community might, therefore, look to the literature on deviance for potential insights into predisposing organizational or societal factors or successful remediation strategies. The deviance literature includes what Currie has termed the "fallacy of autonomy"; the fallacy of autonomy suggests that what goes on in a family, or in this case a hospital or practice, cannot be separated from wider social forces. ${ }^{66}$ Additionally, this literature has described deviant behavior as conduct arising from reactions to personal inability to meet widely accepted goals - something that any clinician who has felt frustrated with perceived barriers to providing good care within the confines of a seemingly under-resourced, overbureaucratized, or excessively profit-driven system can relate to. ${ }^{67}$ Deviant behavior can be moderated by social support - such as, in remediation, a coach or mentor who provides support and validation. ${ }^{68}$ Taking into account the deviance literature might suggest new research questions such as, "What factors in the health care system and/or fee structure encourage the wrong behaviors foster less-than-optimal practices, and/or discourage desired actions?" (rather than, "What individual characteristic caused this problem?); or "Which remediation processes (rather than which plans to re-educate) are more likely to be associated with sustained practice change and a lower rate of recidivism?"

In thinking about which literatures might inform the medical community's thinking around the culture of medicine and how this culture affects the willingness and ability to address remediation, we are struck by how difficult culture change is. ${ }^{69}$ Henri Nouwen wisely wrote "You don't think your way into a new kind of living. You live your way into a new kind of thinking."70 Similarly, we believe that rather than attempting to change the culture of medicine so that the profession can develop more effective remediation programs for practicing physicians, the profession might be more successful by developing better programs to support struggling physicians in order to facilitate needed culture change. 


\section{Conclusion}

As we noted initially, remediation in practicing physicians is a"wicked problem"- a problem that has no simple solutions, a problem that must be grappled with, rather than solved. The specifics of remediation programs will vary depending not only on the particulars of each situation and on the jurisdiction and health care system, but also on, importantly, the underlying conceptualizations of those responsible for their development. We have suggested, generally, that those conceptualizations might benefit from a closer look and, specifically, that viewing remediation as supported practice change and examining its social and systemic aspects might be a good place to start. The remediation of practicing physicians will, however, necessitate more than simply reconceptualizing remediation as behavior change as opposed to filling gaps in knowledge or skills. It will also require a culture change in how the profession views its struggling members, a readiness on the part of institutional stakeholders, especially leaders, to assume responsibility for those aspects of remediation they are best suited to address, and a collective willingness of physicians to support struggling colleagues. The first step going forward will be to explore exactly how these attitudes and culture norms manifest in administrators, those who lead remediation efforts, and those in need of remediation. 


\section{References}

1. Varpio L, Aschenbrener C, Bates J. Tackling wicked problems: How theories of agency can provide new insights. Medical Education 2017;51 (4):353-365.

2. Levac D, Colquhoun $\mathrm{H}, \mathrm{O}^{\prime}$ Brien KK. Scoping studies: Advancing the methodology Implemenation Science 2010;5 (1):69.

3. Cox J. Understanding Doctors' Performance. Oxford: Radcliffe; 2006.

4. Kalet A, Chou CL. Remediation in Medical Education: A Mid-Course Correction. New York: Springer; 2014.

5. Hauer KE, Ciccone A, Henzel TR, et al. Remediation of the deficiencies of physicians across the continuum from medical school to practice: A thematic review of the literature Academic Medicine 2009;84 (12):1822-1832.

6. Price T, Archer J. UK Policy on doctor remediation: Trajectories and challenges. The Journal of Continuing Education in the Health Professions 2017;37 (3):207-211.

7. Lillis S, Takai N, Francis S. Long-term outcomes of a remedial education program for doctors with clinical performance deficits. The Journal of Continuing Education in the Health Professions 2014;34 (2):96-101.

8. Norcross WA, Henzel TR, Freeman KM, Milner-Mares JM, Hawkins RE. Toward meeting the challenge of physician competence assessment: The University of California, San Diego Physician Assessment and Clinical Education (PACE) Program Academic Medicine 2009;84 (8):1008-1014.

9. Goulet F, Jacques A, Gagnon R, Charlin B, Shabah A. Poorly performing physicians: Does the Script Concordance Test detect bad clinical reasoning? The Journal of Continuing Education in the Health Professions 2010;30 (3):161-166.

10. Bhogal HK, Howell E, Torok H, Knight AM, Howell E, Wright S. Peer assessment of professional performance by hospitalist physicians. Southern Medical Journal 2012;105 (5):254-258.

11. Grace ES, Wenghofer EF, Korinek EJ. Predictors of physician performance on competence assessment: Findings from CPEP, the Center for Personalized Education for Physicians. Academic Medicine 2014;89 (6):912-919.

12. Chesluk BJ, Reddy S, Hess B, Bernabeo E, Lynn L, Holmboe E. Assessing interprofessional teamwork: Pilot test of a new assessment module for practicing physicians. The Journal of Continuing Education in the Health Professions 2015;35 (1):3-10.

13. Haddad T. Cognitive assessment in the practice of medicine-dealing with the aging physician. Physician Executive 2013;39 (4):14-20.

14. Donnon T, Al Ansari A, Al Alawi S, Violato C. The reliability, validity, and feasibility of multisource feedback physician assessment: A systematic review. Academic Medicine 2014;89 (3):511-516.

15. van der Vleuten CP, Schuwirth LW, Scheele F, Driessen EW, Hodges B. The assessment of professional competence: Building blocks for theory development. Best Practices and Research Clinical Obstetrics \& Gynacology 2010;24 (6):703-719.

16. Miller A, Archer J. Impact of workplace based assessment on doctors' education and performance: A systematic review. BMJ 2010;341 c5064.

17. Hawkins R, Roemheld-Hamm B, Ciccone A, Mee J, Tallia A. A multimethod study of needs for physician assessment: Implications for education and regulation The Journal of Continuing Education in the Health Professions 2009;29 (4):220-34.

18. Leape LL, Fromson JA. Problem doctors: Is there a system-level solution? Annals of Internal Medicine 2006;144 (2)107-115. 
19. Williams BW. The prevalence and special educational requirements of dyscompetent physicians. The Journal of Continuing Education in the Health Professions 2006;26 (3):173-191.

20. Goulet F, Jacques A, Gagnon R. An innovative approach to remedial continuing medical education, 1992-2002. Academic Medicine 2005;80 (6):533-540.

21. Rosenblatt MA, Abrams KJ, New York State Society of Anesthesiologists, Inc, Committee on Continuing Medical Education, Remediation Sub-Committee. The use of a human patient simulator in the evaluation of and development of a remedial prescription for an anesthesiologist with lapsed medical skills. Anesthesia \& Analgesia 2002;94 (1)149-153.

22. Rhydderch $M$, Matthews $P$, Beech $M$. The advanced training practice network: Providing rescribed further training for general practitioners in Wales. Education for Primary Care 2007;18 (5):572-581.

23. Cassel CK, Holmboe ES. Professionalism and accountability: The role of specialty board certification. Transactions of the American Clinical and Climatological Association 2008;1 19:295303.

24. Goulet F, Gagnon R, Gingras ME. Influence of remedial professional development programs for poorly performing physicians. The Journal of Continuing Education in the Health Professions 2007;27 (1):42-48.

25. Wenghofer EF, Way D, Moxam RS, Wu H, Faulkner D, Klass DJ. Effectiveness of an enhanced peer assessment program: Introducing education into regulatory assessment. The Journal of Continuing Education in the Health Professions 2006;26 (3):199-208.

26. Bleakley A, Bligh J, Browne J. Medical Education for the Future: Identity, Power and Location, vol. 1. Dordrecht, Netherlands: Springer; 2011

27. Norton PG, Ginsburg LS, Dunn E, Beckett R, Faulkner D. Educational interventions to improve practice of nonspecialty physicians who are identified in need by peer review. The Journal of Continuing Education in the Health Professions 2004;24 (4):244-252.

28. Parran TV Jr, Pisman AR, Youngner SJ, Levine SB. Evolution of a remedial CME course in professionalism: Addressing learner needs, developing content, and evaluating outcomes. The Journal of Continuing Education in the Health Professions 2013;33 (3):174-179.

29. Kennedy T, Regehr G, Rosenfield J, Roberts SW, Lingard L. Exploring the gap between knowledge and behavior: A qualitative study of clinician action following an educational intervention. Academic Medicine 2004;79 (5):386-393.

30. Mazmanian PE, Daffron SR, Johnson RE, Davis DA, Kantrowitz MP. Information about barriers to planned change: A randomized controlled trial involving continuing medical education lectures and commitment to change. Academic Medicine 73 (8):882-886.

31. Lingard L. Rethinking competence in the context of teamwork. In Hodges BD, Lingard L, eds. The question of competence: Reconsidering medical education in the twenty-first century. Ithaca: Cornell University 2012: p.42-69.

32. Smith R. Remediation Working Group Report. London: Academy of Medical Royal Colleges 2012.

33. Humphrey C. Assessment and remediation for physicians with suspected performance problems: An international survey. The Journal of Continuing Education in the Health Professions 2010;30 (1):26-36.

34. Bosk CL. Forgive and Remember: Managing Medical Failure. Chicago: University of Chicago Press; 2003.

35. Mutabdzic $D$, Mylopoulos $M$, Murnaghan ML, et al. Coaching surgeons: Is culture limiting our ability to improve? Annals of Surgery 2015;262 (2):213-216.

36. Ciccarelli SK, White JN. Psychology, 4th edition. Boston: Pearson Education Inc.; 2015. 
37. Albanese M, Mejicano G, Xakellis G, Kokotailo P. Physician practice change I: A critical review and description of an Integrated Systems Model. Academic Medicine 2009;84 (8) :1043-1055.

38. Marinopoulos SS, Dorman T, Ratanawongsa N, et al. Effectiveness of Continuing Medical Education. Evidence Report/Technology Assessment No. 149 (Prepared by the Johns Hopkins Evidence-based Practice Center) Rockville, MD: Agency for Healthcare Research and Quality; January 2007. https://archive.ahrq.gov/downloads/pub/evidence/pdf/cme/cme.pdf. Accessed April 5, 2018.

39. Eve R, Golton I, Hodgkin P, Munro J, Musson G. Beyond guidelines: Promoting clinical change in the real world. Journal of Management in Medicine 1996;10 (1):16-25.

40. General Practice Services Committee. Practice Support Program. http://www.gpscbc.ca/whatwe-do/professional-development/psp/group-learning-sessions. Accessed April 5, 2018.

41. Olson CA. Twenty predictions for the future of CPD: Implications of the shift from the update model to improving clinical practice. The Journal of Continuing Education in the Health Professions 2012;32 (3):151-152.

42. Shershneva MB, Harper PL, Elsinger LM, Olson CA. Facilitating multiorganizational smoking cessation knowledge translation through on-line toolkit for educators and clinicians. The Journal of Continuing Education in the Health Professions 2010;30 (2):149-150.

43. Hodges B. The shifting discourses of incompetence. In Hodges BD, Lingard L, eds. The question of competence: Reconsidering medical education in the twenty-first century. Ithaca: Cornell University; 2012: 14-41.

44. Papadakis MA, Hodgson CS, Teherani AP, Kohatsu ND. Unprofessional behavior in medical school is associated with subsequent disciplinary action by a state medical board. Academic Medicine 2004;79 (3):244-249.

45. Roback HB, Strassberg D, lannelli RJ, Finlayson AJ, Blanco M, Neufeld R. Problematic physicians: A comparison of personality profiles by offence type. Canadian Journal of Psychiatry 2007;52 (5):315-322.

46. Samenow CP, Yabiku ST, Ghulyan M, Williams B, Swiggart W. The role of family of origin in physicians referred to a CME course. HEC Forum 2012;24 (2):115-126.

47. Gabbay J, le May A. Practice made perfect: Discovering the roles of a community of general practice. In le May A, ed. Communities of Practice in Health and Social Care. Malden MA: WileyBlackwell; 2009: 49-65.

48. Ross L. The intuitive psychologist and his shortcomings: Distortions in the attribution process. Advances in Experimental Social Psychology 1977;10:173-220.

49. Lerner MJ, Miller DT. Just world research and the attribution process: Looking back ahead. Psychological Bulletin 1978;85 (5):1030-1051.

50. Gino F, Moore DA, Bazerman MH. No Harm, no foul: The outcome bias in ethical judgments. Harvard Business School Working Paper No 08-080. https://www.hbs.edu/faculty/ Publication\%20Files/08-080_1751f2c7-abe2-402b-9959-1d8190ebf62a.pdf Accessed April 5, 2018.

51. van der Vleuten $C$. Validity of final examinations in undergraduate medical training. $B M J$ 2000;321 (7270):1217-1219.

52. Dijksterhuis MG, Voorhuis M, Teunissen PW, et al. Assessment of competence and progressive independence in postgraduate clinical training. Medical Education 2009;43 (12):1156-1165.

53. Epstein RM. Assessment in medical education. New England Journal of Medicine 2007;356 (4):387-396. 
54. Postuma RA, Campion MA. Age stereotypes in the workplace: Common stereotypes, moderators, and future research directions. Journal of Management 2009;35 (1):158-188.

55. Collins ME, Block SD, Arnold RM, Christakis NA. On the prospects for a blame-free medical culture. Social Science \& Medicine 2009;69 (9):1287-1290.

56. Silversin J, Kornacki MJ. Culture and Compact. Karnacki MJ, Silversin J. Leading Physicians Through Change: How to Achieve and Sustain Results. Tampa, Florida: American College of Physician Executives; 2000:47.

57. Chamberlain JM. Sociological deconstructions I: Critiquing medical autonomy and altruism. In Chamberlain JM. The Sociology of Medical Regulation: An Introduction. Dordrecht, Netherlands: Springer; 2012:69-92.

58. Friedson E. The characteristics of a profession. In: Earle $\mathrm{S}$ and Letherby $\mathrm{G}$, ed. The Sociology of Health Care: A reader for Health Professionals. Hampshire UK: Palgrave Macmillan; 2008:247-257.

59. Merriam SB. Andragogy and self-directed learning: Pillars of adult learning theory. In New directions for adult and continuing education 2001;89:3-13.

60. Norman GR. The adult learner: A mythical species. Academic Medicine 1999;74 (8):886-889.

61. Kolb DA, Boyatzis RE, Mainemelis C. Experiential learning theory: Previous research and new directions. The educational psychology series: Perspectives on thinking, learning, and cognitive styles. 2001;1 (8):227-247.

62. Schön DA. Educating the Reflective Practitioner: Toward a New Design for Teaching and Pearning in the Professions. San Francisco, CA: Jossey-Bass; 1987.

63. Smith WR. Evidence for the effectiveness of techniques to change physician behavior. Chest 2000;118 (2):8S-17S.

64. Engeström Y. Expansive learning at work: Toward an activity theoretical reconceptualization. Journal of Education at Work 2001;14 (1):133-156.

65. Grenny J, Patterson K, Maxfield D, McMillan R, Switzler A. Influencer:The New Science of Leading Change, 2nd ed. New York: McGraw Hill Professional; 2013.

66. Currie E. Confronting Crime: An American Challenge. New York: Pantheon Books; 1985.

67. Merton RK. Social structure and anomie. American Sociological Review 1938;3 (5):672-682.

68. Cullen FT. Social support as an organizing concept for criminology: Presidential address to the Academy of Criminal Justice Sciences. Justice Quarterly 1994;11 (4):527-559.

69. Fein IA, Corrato RR. Clinical practice guidelines: Culture eats strategy for breakfast, lunch, and dinner. Critical Care Medicine 2008;36 (4):1360-1361.

70. Nouwen HJM. Introduction to the 1980 edition. In Palmer PJ. The Promise of Paradox: A Celebration of Contradictions in the Christian Life. Notre Dame, Ind: Ave Maria Press; 1980. 


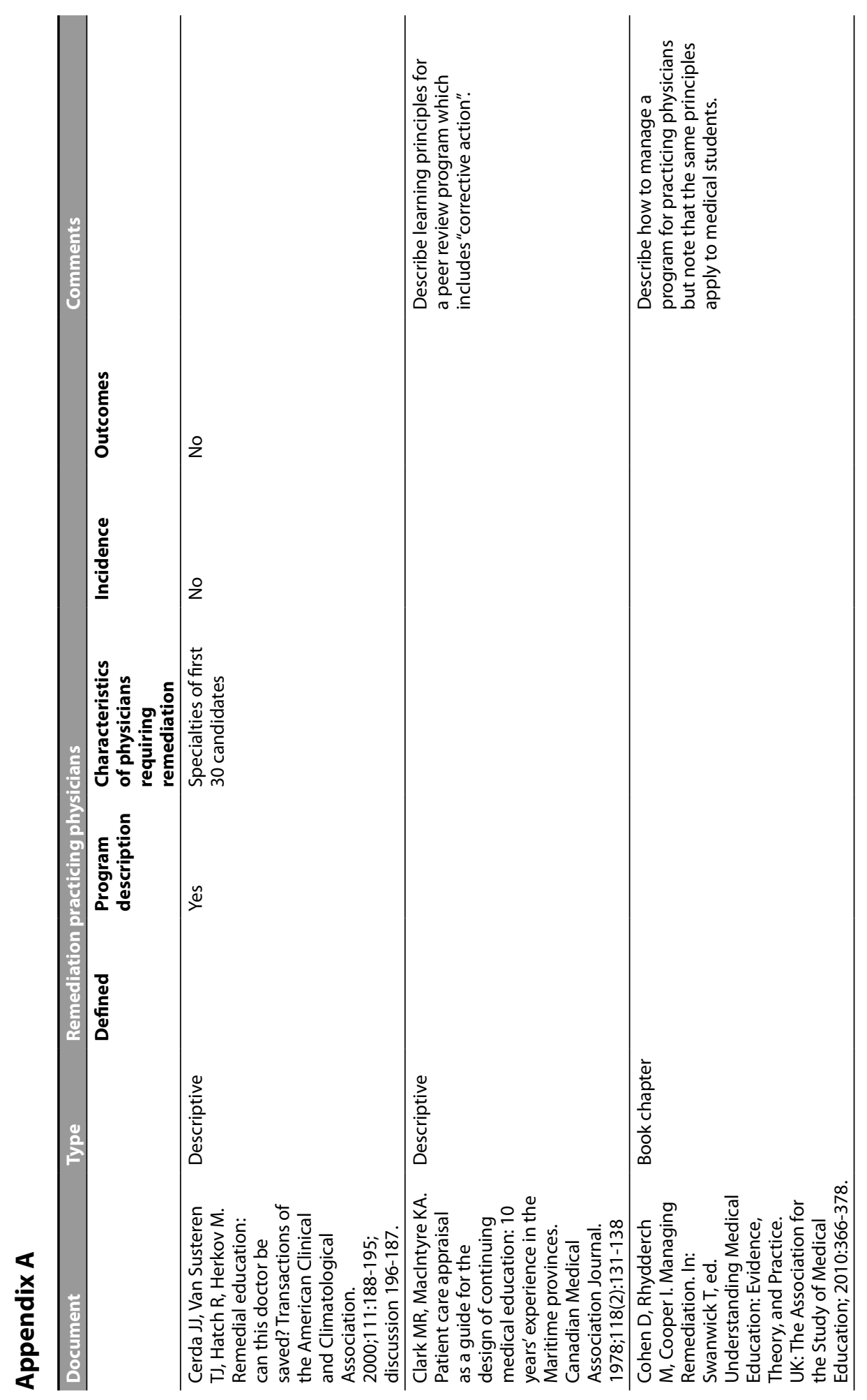




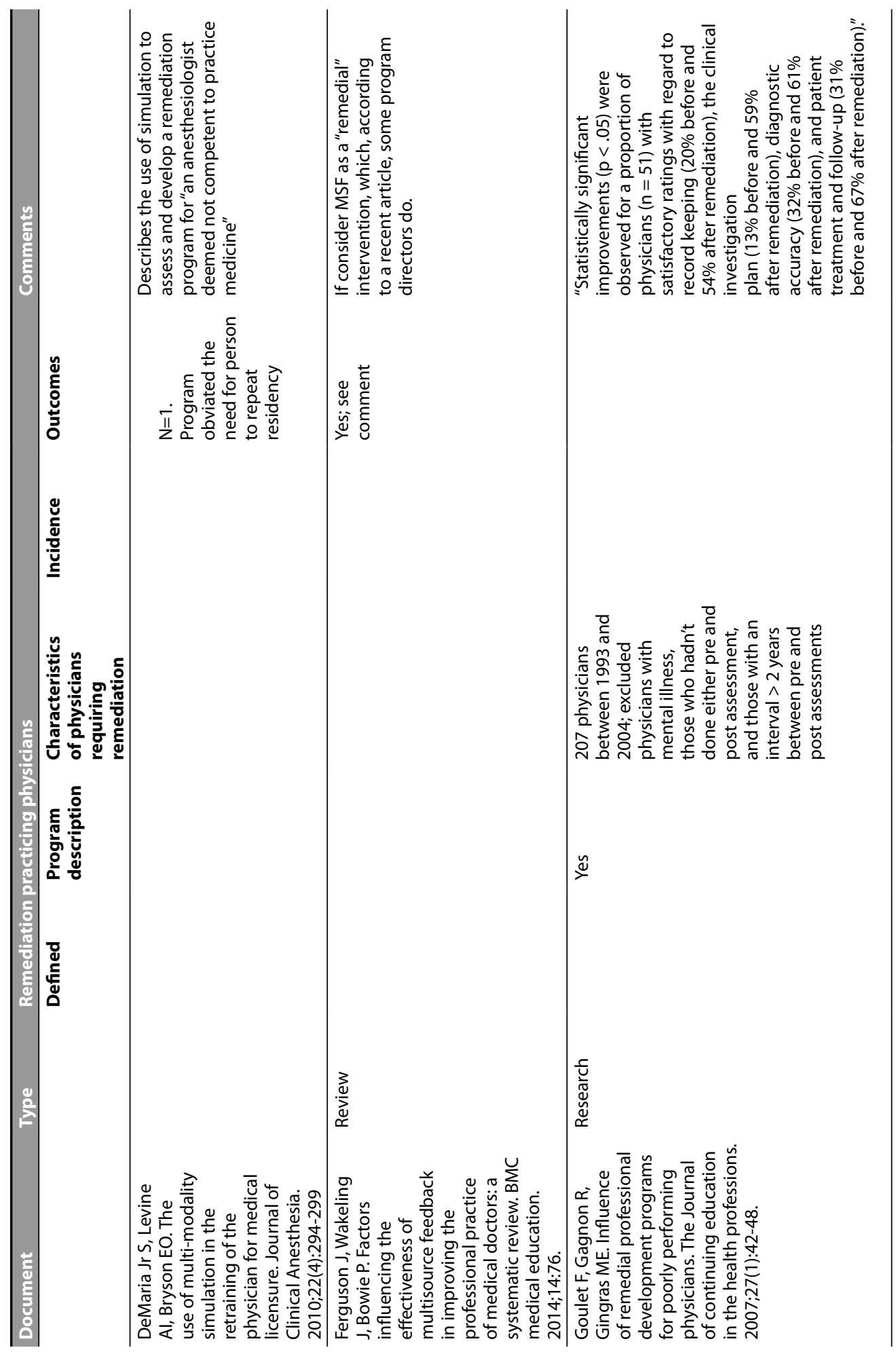




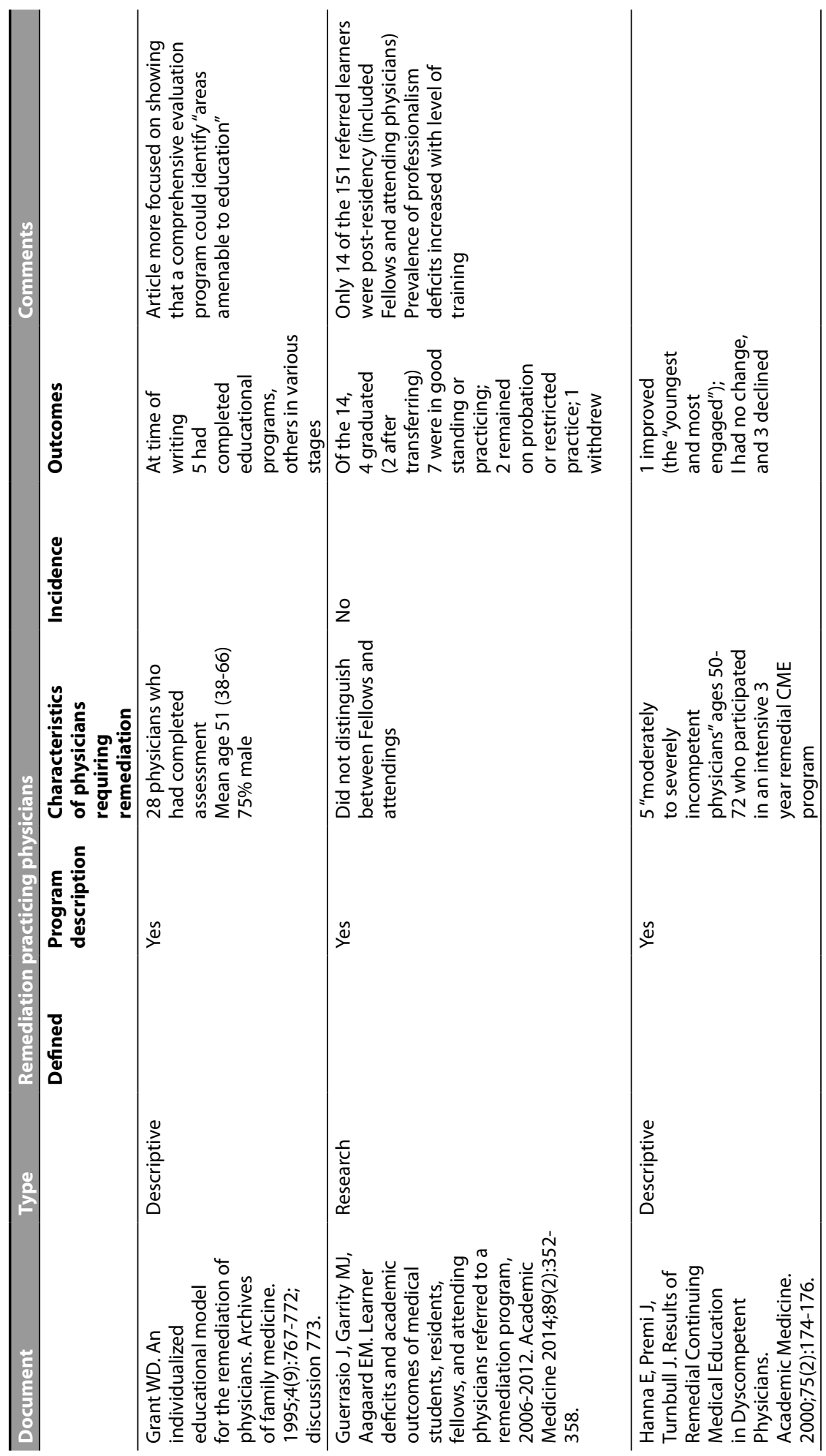



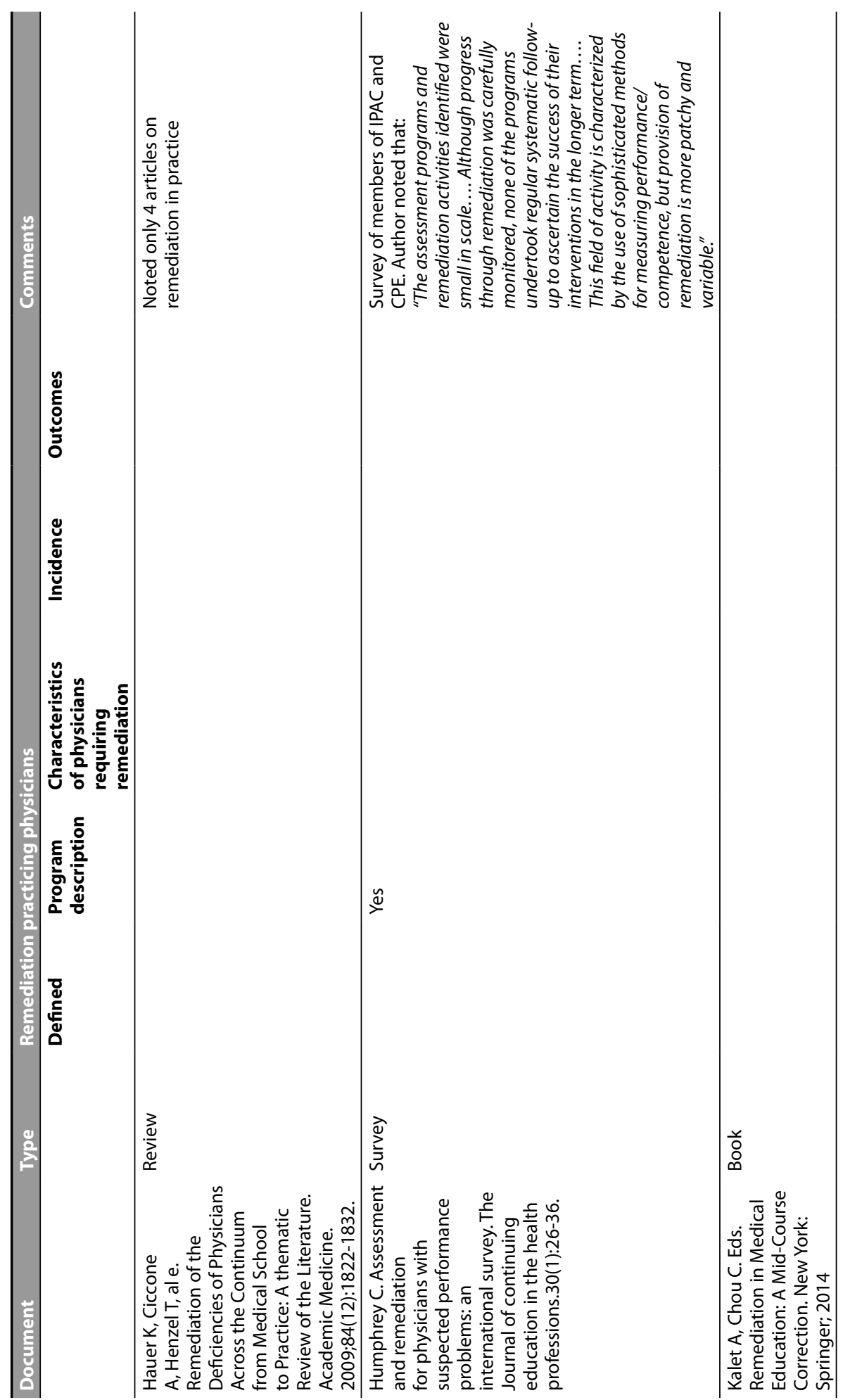


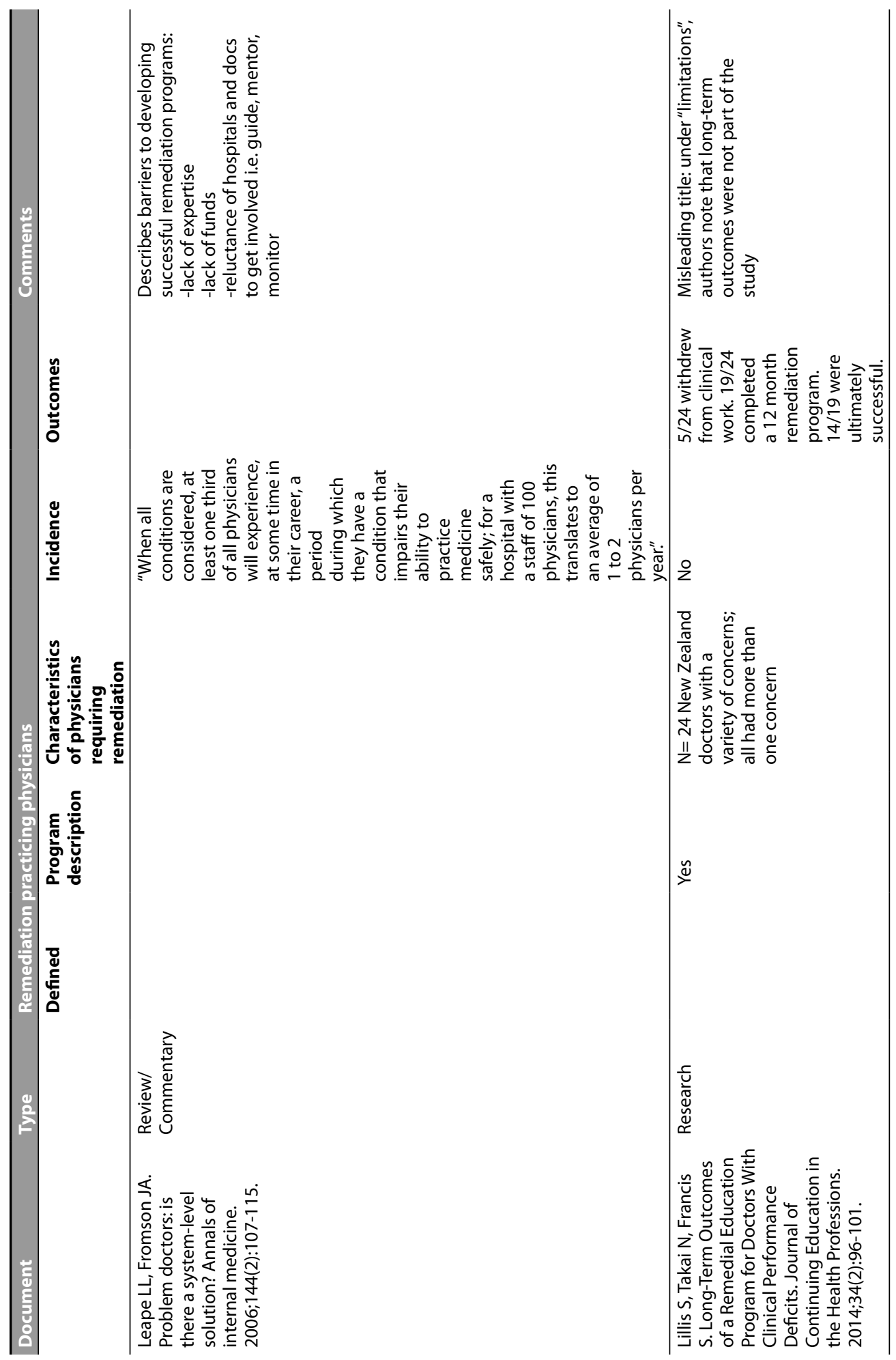




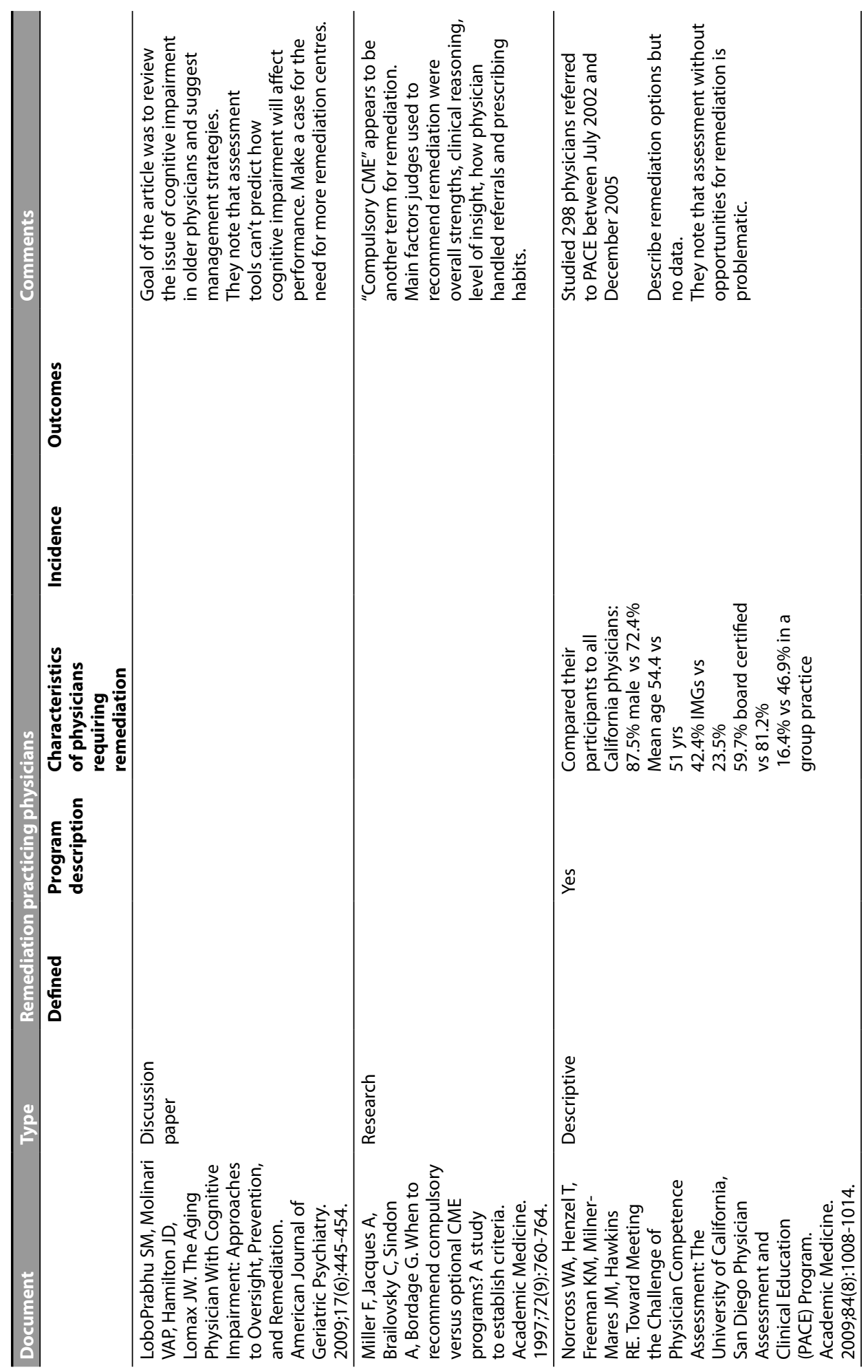




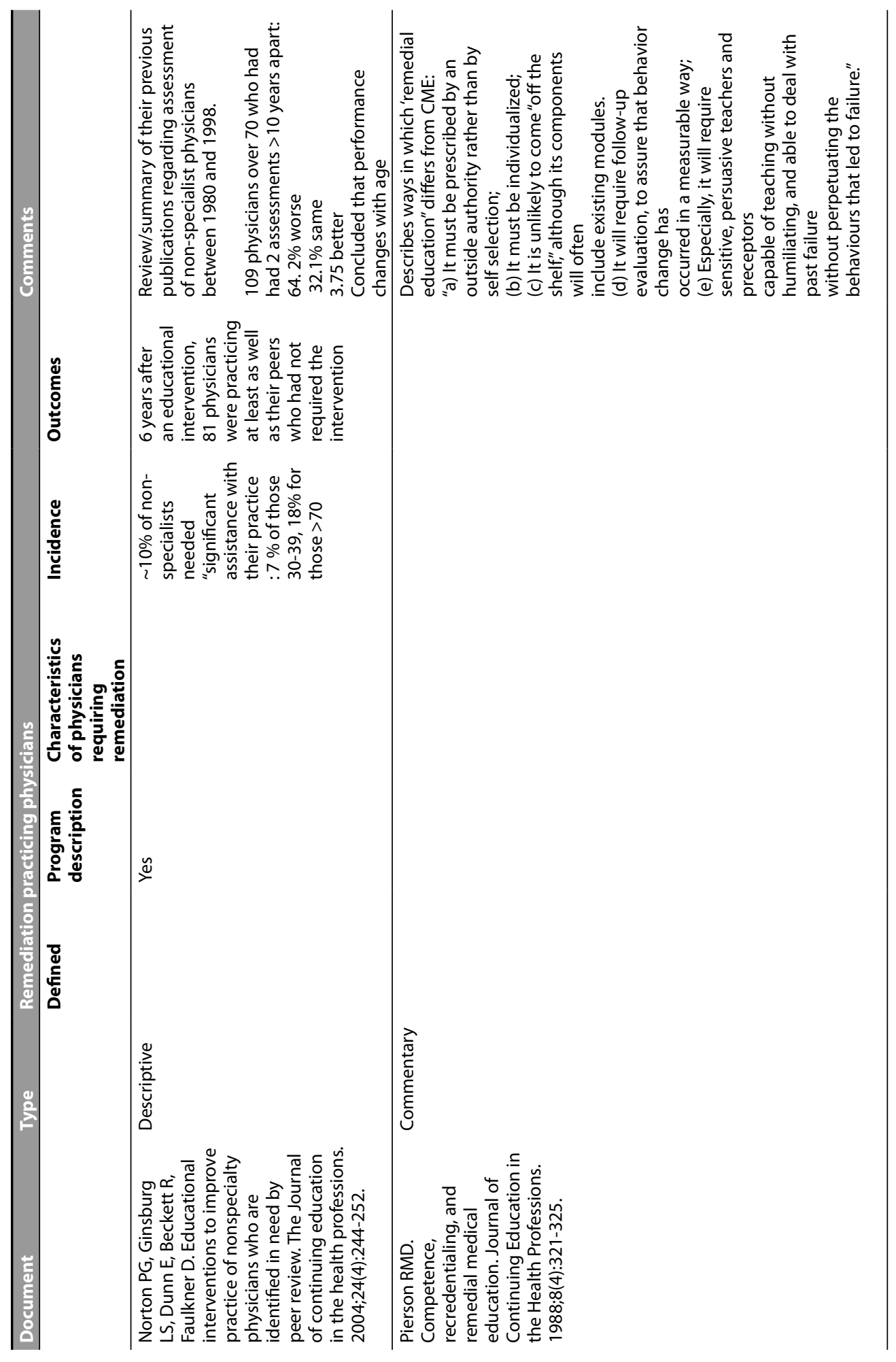




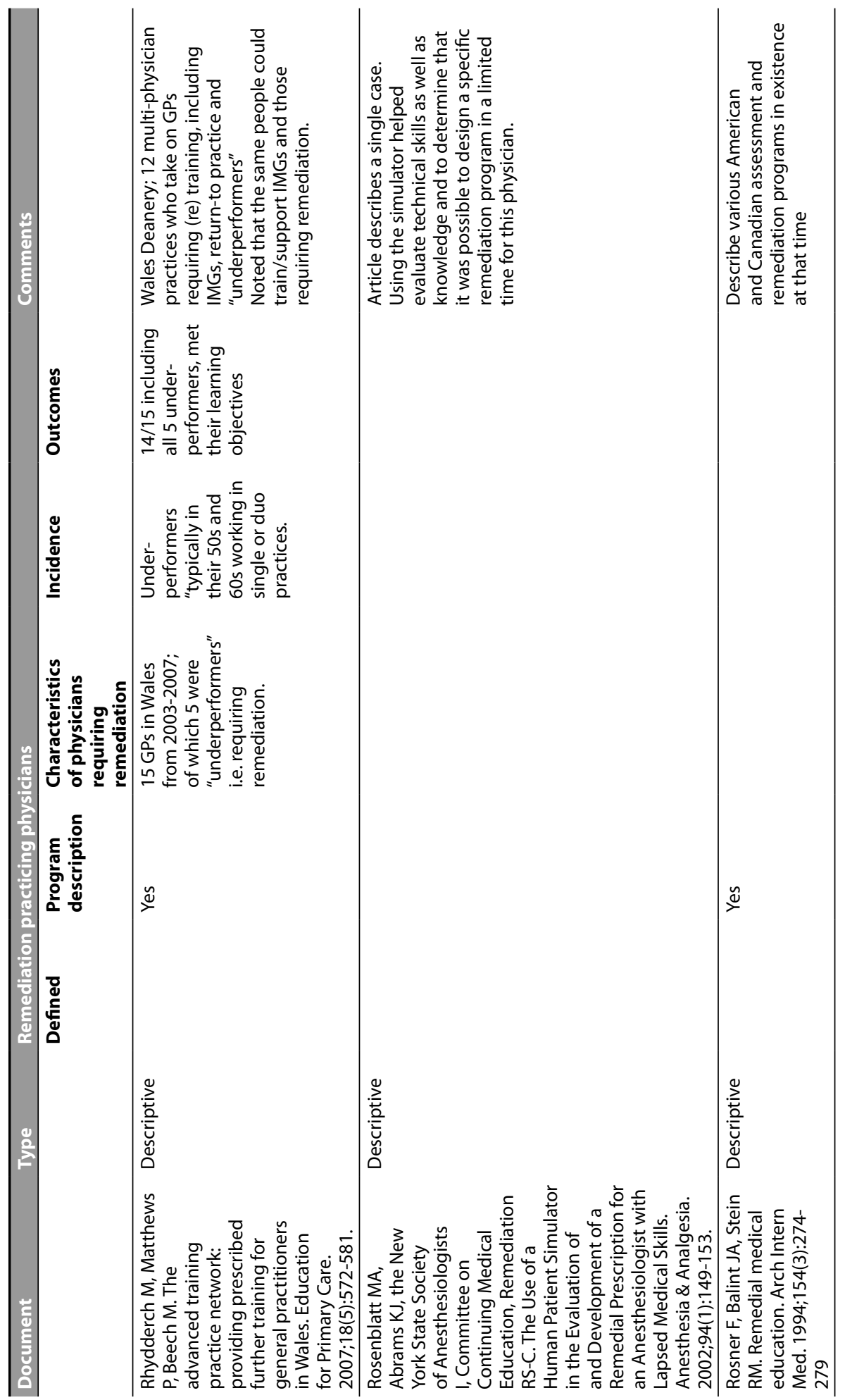




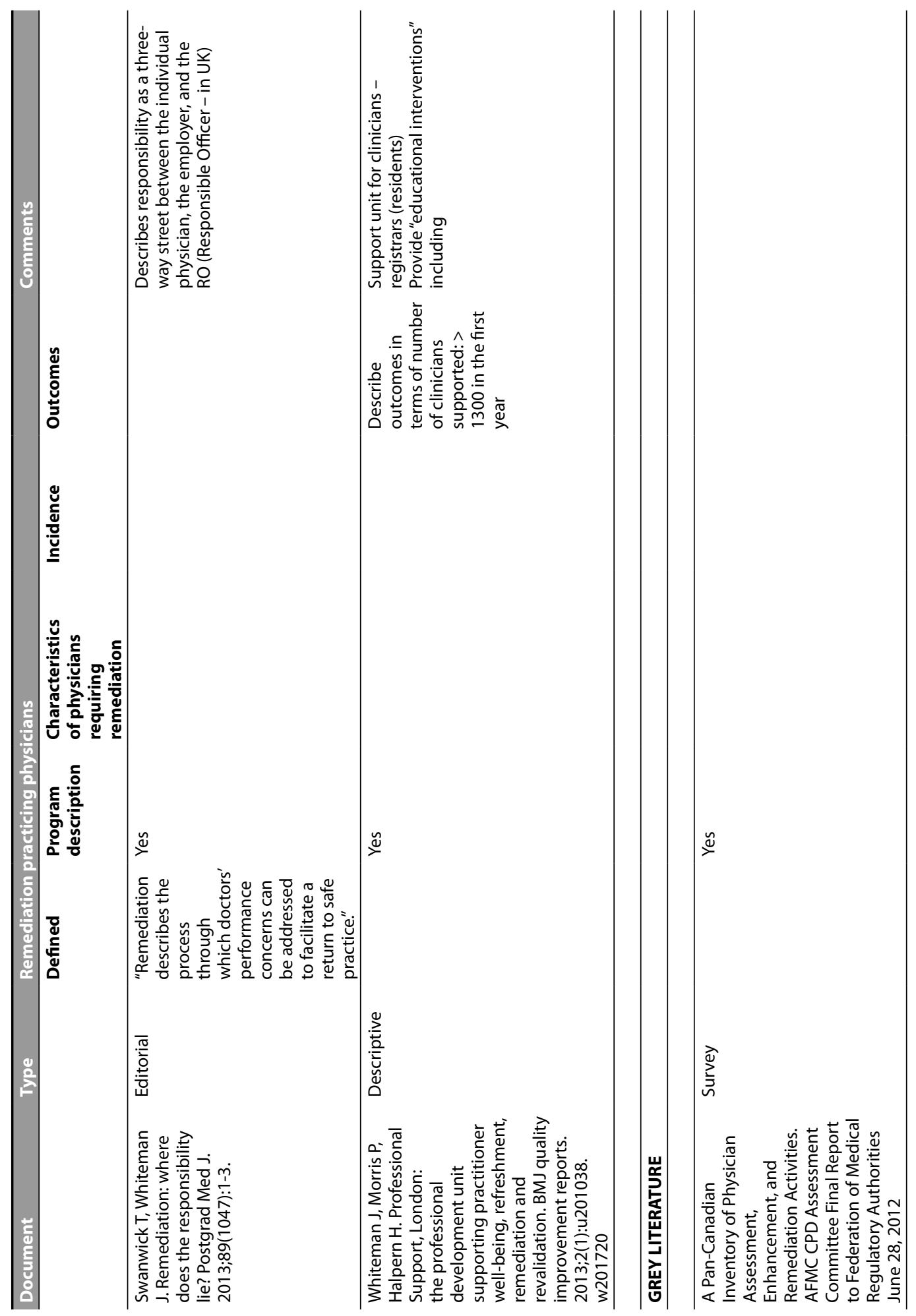




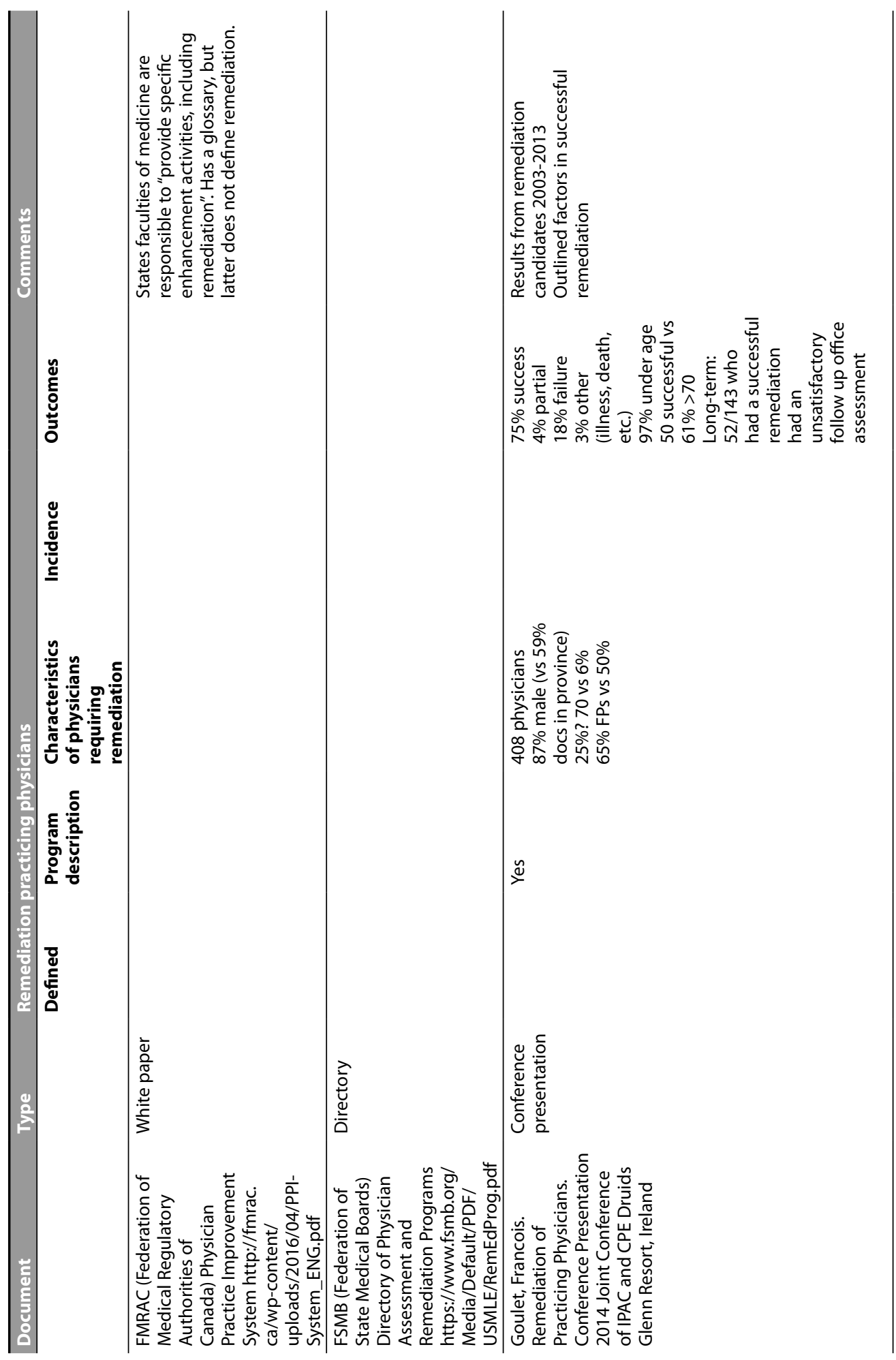




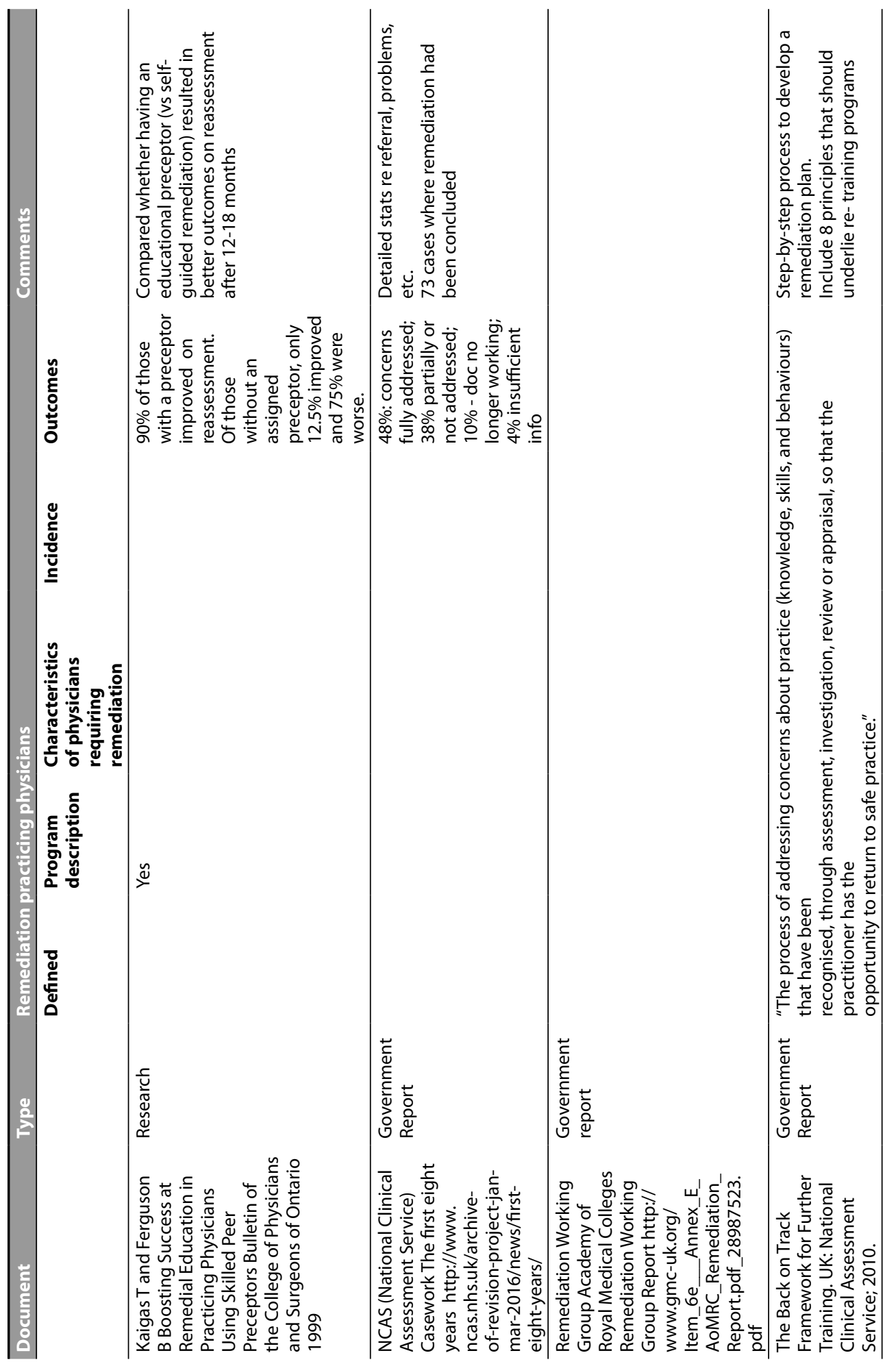




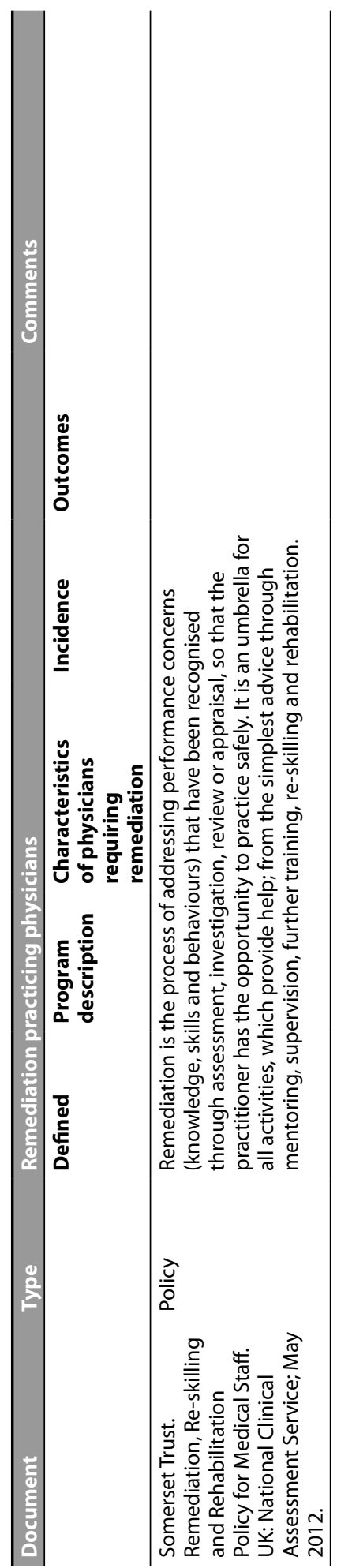




\section{Education or Regulation? Exploring our Underlying Conceptualisations of Remediation for Practising Physicians}

Gisèle Bourgeois-Law | Lara Varpio, | Glenn Regehr | Pim W Teunissen 


\section{ABSTRACT}

Context The remediation of practising physicians is coming to the fore in several countries in response to increasing demands for physician accountability and quality improvement initiatives in health care. However, the profession continues to grapple with the concepts and processes of remediation, particularly for physicians in practice who struggle with performance issues related to clinical competence. This suggests that current conceptualisations of remediation might be contributing to this situation. This study therefore, explored how various administration-level stakeholders conceptualise remediation in order to construct a description of the nature and meaning of remediation.

Methods Semi-structured interviews were conducted using purposive sampling across the range of Canadian stakeholders involved in the remediation of practising physicians, including regulatory authorities, universities, national certifying bodies and medical organisations. With the use of constructivist grounded theory processes, analysis proceeded apace with data collection in an iterative process, with initial insights guiding subsequent interviews.

Results Participants often simultaneously held two different conceptualisations of remediation: (i) remediation as part of an educational continuum involving different degrees of support, and (ii) remediation as a regulatory process removing an individual's educational autonomy. Interviewees moved between these two conceptualisations but did not always appear to be aware of doing so. These conceptualisations each had different implications regarding the degree to which remediation can be incorporated into professional processes of maintaining competence.

Conclusion Understanding that stakeholders frequently approach the complex issue of remediation with two different perspectives without conscious awareness of doing so may help to explain several challenges in the field, including the issues of what falls under the umbrella of remediation and who should be responsible for offering remediation support. Our findings suggest the need for conceptual clarity around remediation, both to ensure that we illuminate logistical dilemmas in enacting remediation and to address the stigma of 'deprofessionalisation' that the provision of even minor educational supports (such as feedback) might invoke by association. 


\section{INTRODUCTION}

The remediation of practising physicians is coming to the fore in several countries in response to increasing demands for physician accountability and quality improvement initiatives in health care. ${ }^{1-4}$ However, with few exceptions, mechanisms to support physician remediation (i.e. bringing unsatisfactory performance up to an expected standard) remain largely underdeveloped..$^{5-7}$ Even in jurisdictions with established programmes, significant challenges persist, including those associated with competition for training resources with other learners, the resolution of concerns of legal liability, and the determination of which organisations are best suited to taking responsibility for invoking, enacting and monitoring the remediation of practising physicians. ${ }^{8}$ It seems that the ability to identify physician performance concerns continues to outpace the ability to redress them. ${ }^{9}$ This undoubtedly contributes to the stress experienced by the practitioners identified, which may further impact performance and may contribute to pushback from the medical profession with regard to revalidation in that physicians may respond that it is 'unfair' to tell someone that aspects of his or her practice are problematic when failing to provide the means to address the issue. Moreover, it may lead to increased legal risk for regulatory and health authorities if a serious error were to be committed by a physician previously identified as underperforming.

One reason that remediation continues to be a wicked problem may refer to the fact that remediation has tended to be constructed as an education issue, yet few remediation studies explicitly articulate an underlying education theory that informs this construction. ${ }^{10,11}$ For physicians in practice, individual learning theories have traditionally been more popular in continuing professional development (CPD), but are rarely and only indirectly referred to in remediation papers. ${ }^{12,5,7}$ Only recently have authors started to move beyond strictly educational conceptualisations and to suggest the use of behavioural change theories to develop a remediation model. ${ }^{13}$

Perhaps a more important issue in the persistence of remediation as a wicked problem lies in its personal, professional and collective implications. We have suggested elsewhere that deeper social and psychological issues may be at play, confounding our efforts to solve the problem, particularly that of professional autonomy. ${ }^{14}$ The literature on what it means to be a professional and on professional autonomy suggests that autonomy is the defining characteristic of a professional, which would imply that remediation may be fraught because it interferes with individual clinician autonomy and, by extension, with professional status. ${ }^{15,16-18}$ Even more important than its threat to stature is its potential challenge of an individual's very being in terms of his or her identity as a (medical) professional. These two aspects of remediation, its educational construction and its potential impact on professional status and identity, informed the conceptual orientation of this study.

As the profession continues to struggle with logistics around remediation, shifting the focus to how remediation is conceptualised might provide further insights into the challenges of implementing it well.,13,19-21 Thus, as part of a larger programme of research that aims to construct a more comprehensive, consistent and nuanced understanding 
of the nature and meaning of remediation, this study explored the conceptualisations of remediation held by representatives from a variety of institutions that are seen as having some stake in the process of remediation in Canada.

\section{METHODS}

We chose to use constructivist grounded theory (CGT) for this study because, unlike classical grounded theory which is rooted in positivist and post-positivist orientations, CGT rests on assumptions that social reality is the product of interpretations. ${ }^{22,23}$ This perspective is aligned with our study goals of constructing, with key stakeholders, a nuanced description of the nature and meaning of remediation. Constructivist grounded theory recognises that 'personal and professional background, plus experience, provide positive forces for the analysis process, thereby partly providing necessary theoretical sensitivity.22 Thus, just as it is essential to the success of this study to understand our participants' subjectivity, we are also aware that our own experiences inform this construction work.

Each member of our research team brings a unique perspective to this research endeavour. GB-L is a physician with past experience as the director of a physician assessment and enhancement programme. LV is a PhD-trained qualitative research scientist who examines how individuals, teams, organisations and fields co-construct one another. GR is a PhD trained researcher with over 25 years of experience in health professions education scholarship. PWT is an obstetrician working in tertiary care and a researcher in health professions education with a particular focus on workplace learning. The research team's backgrounds and experiences shaped the research questions posed, the data collected and the interpretations created.

\section{Participants and sampling}

Regulation is variously organised in different countries and often within countries. In Canada, medical regulation and health care are provincial responsibilities. A need for remediation is generally determined either by a provincial regulatory authority or, less often, by a regional health authority. Once remediation needs are determined, the actual remediation might be carried out by: (i) preceptors identified by the regulatory or health authority; (ii) preceptors identified by the authority, or (iii) university CPD offices or postgraduate programmes. There is wide variability in the practices adopted by the various provinces. Consequently, the Canadian context seemed suitable to study our research questions because this variability allowed us to sample for maximum variation in terms of participants' working conditions. The study began by using purposive sampling to recruit Canadian remediation stakeholders, or individuals in organisations that have, or might be expected to have, some degree of responsibility for the remediation of practising physicians. After the first 10 interviews, we transitioned from purposive to theoretical sampling, inviting additional interviewees who could help to confirm or disprove elements of our descriptions and developing theory. For example, the insights offered by an interviewee who had been both a postgraduate (residency) dean and a department head helped us to determine that we needed to interview at least one other department head, as well as an individual involved in quality improvement within a health authority. 
In total, we interviewed 17 participants. In keeping with CGT methodology, we stopped recruiting participants when we had achieved saturation, represented by the point at which our sample size was large enough to provide the data needed to develop new knowledge around the study phenomenon, and no new concepts or considerations were being offered by participants. Participant characteristics are outlined in Table 1. Several of our participants, although not directly involved in remediation, had past experience in related areas (e.g. two of our participants had been, at one time, directors of physician assessment programmes). Because of her previous experience in working in the physician remediation field, GB-L, the principal investigator, knew several of the participants. This may have influenced individuals' willingness to participate and their openness in interviews.

\begin{tabular}{|c|c|}
\hline \multicolumn{2}{|l|}{ PARTICIPANT DEMOGRAPHICS } \\
\hline PROVINCE & $\begin{array}{l}\text { Alberta }-1 \\
\text { British Columbia - } 4 \\
\text { Manitoba }-2 \\
\text { National }-2 \text { (Certifying Colleges) } \\
\text { Ontario }-5 \\
\text { Québec }-2 \\
\text { Nova Scotia }-1\end{array}$ \\
\hline $\begin{array}{l}\text { ROLES } \\
\text { ( } n>17 \text { as some participants had more than one role) }\end{array}$ & $\begin{array}{l}\text { Certifying Colleges - } 2 \\
\text { CPD or former CPD Deans - } 5 \\
\text { Deans - } 1 \\
\text { Department Heads - } 2 \\
\text { Health Authorities - } 1 \\
\text { PGME Deans - } 3 \\
\text { Physician Health Program - } 1 \\
\text { Regulatory Authorities - } 4\end{array}$ \\
\hline Declined or did not answer & $\begin{array}{l}\text { CPD - } 3 \\
\text { Deans }-3 \\
\text { Medical Associations - } 1 \\
\text { National - } 1 \\
\text { PGME - } 2 \text { (but } 1 \text { referred to colleague in another } \\
\text { university who participated) }\end{array}$ \\
\hline
\end{tabular}

${ }^{*} \mathrm{n}=>17$ as some participants had more than one role.

$\mathrm{CPD}=$ continuing professional development;

$\mathrm{PGME}=$ postgraduate medical education

\section{Interviews}

GB-L conducted 1-hour, semi-structured interviews with participants in person $(n=7)$ or by telephone $(n=10)$, depending on the participant's geographical proximity. These interviews were audiorecorded, transcribed by a third-party service, and anonymised during the transcription process.

To ensure the clarity of interview prompts and their alignment with participants' interpretations of the prompts, GBL piloted the interview with one stakeholder. Interview questions explored how participants conceptualised the remediation of practising physicians, how they viewed physicians requiring remediation, and the political, educational and sociocultural considerations surrounding the issue of physician remediation. Questions were open-ended and made no reference to education, autonomy 
or other concepts that might direct participants' responses. As per CGT methodology, questions evolved over the course of the study so that, for example, when a participant mentioned the lack of any checklist to determine when an individual should be sent for remediation, in future interviews we explored how that decision was made. A list of the interview questions is included in Appendix S1.

All interviews were carried out in the participant's primary language (i.e. either English or French, Canada's two national languages). Two interviews were conducted in French and then translated into English. To confirm this translation, the English documents were translated back into French by a different translator. GB-L then searched the two French documents for instances in which the meanings of the transcribed texts differed and went back to the translators when necessary to ensure that we were correctly capturing the participants' expressions. The research team worked with all transcripts in English.

\section{Analysis}

Interviews were coded in an ongoing, iterative manner as data collection progressed. Analytic memos were written by GB-L throughout data collection and analysis. ${ }^{22} \mathrm{We}$ revised the interview protocol as the study progressed to vet and challenge the themes we were developing with interviewees. GB-L coded all interviews, and LV reviewed portions of the coding to confirm a shared understanding and application of the themes. The research team met via telephone, video conference and in person to discuss coding and to construct the theoretical framework. We used the qualitative software DEDOOSE TM (SocioCultural Research Consultants, LLC, Manhattan Beach, CA, USA) to support analysis because it enabled our team to review the study data and collaborate on analysis across national borders in a secure manner.

The study received ethical approval from the University of British Columbia Behavioral Research Ethics Board (protocol no. H16-00529).

\section{RESULTS}

Our primary finding was that participants appeared to hold two different, potentially conflicting, conceptualisations of remediation. From one perspective, they saw remediation as an educational endeavour, different in degree but not in kind from continuing medical education (CME) and CPD. By contrast, participants also viewed remediation as an imposed regulatory process that involved the removal of educational autonomy, meaning the individual was no longer able to choose when, what and how much to learn, nor to independently determine when learning had occurred. These conceptualisations each had different implications regarding the acceptability of remediation and its place in the profession.

\section{Remediation as an educational continuum}

Participants conceptualised remediation, first and foremost, as a form of supported education. In elaborating this construction of remediation, participants emphasised that virtually every practitioner requires some degree of external support to practise 
optimally. They recognised that 'in one sense, we're all remediating something' (CPD 5). This perception that remediation is synonymous with a degree of supported learning was clearly part of participants' understanding of the broadly scoped nature of the phenomenon:

I just think it's all part of the spectrum ... when physicians acquire new skills or enhance their understanding of disease processes or pathology based on, you know, new theory or clinical trials, I mean, in one sense they are remediating their practice. To remediate is to change. (Certifying college [CC] 2)

Although these descriptions suggest that any form of supported learning might be considered as remediation, there was also a recognition of a continuum, defined by, firstly, the size of the knowledge, skill or attitude gap a physician might need to fill and, secondly, the amount of support needed to address the gap. Participants acknowledged that, although conceptually 'we're all remediating', functionally the term 'remediation' was a label reserved for the far end of the continuum. However, the point at which an individual would land in remediation was somewhat arbitrary. In other words, somewhere along this continuum, learning changes from a continuum of supported practice improvement effort in which all physicians need to engage to a formal remediation endeavour that aims to address a significant or problematic gap in a physician's expertise. This was explicitly articulated by one participant:

It's a continuum. We all have things in our work that we do well and things that we do not do so well. You know: nobody's perfect. Nobody's practice does not need improvement. And there may be some people whose practice needs more improvement, and those [are] the ones we call remediation. (Regulatory Authority $[R A]$ 1)

Framing remediation as the arbitrary application of a term at some point along the educational support continuum has an important implication: although there may be some stigma attached to an individual who needs more support than an average physician, remediation should not be viewed negatively. In fact, one participant argued that 'savvy' physicians would actually seek out the experience labelled 'remediation':

[A colleague] made a really interesting comment about that. She said, you know: "Really savvy doctors would look for remediation opportunities 'cause it's going to be a far better educational opportunity than any sort of CME they're going to get." And I thought that's such an interesting thing to say and she's right. Because that's a rich educational experience. I'd probably be a better practitioner if my peers came in and sat in my clinic with me and watched what I do. And that very seldom happens unless you're in a remediation setting. (CPD 1)

When conceived of in this way, remediation becomes problematic only when the educational resources required exceed the available or commonly used resources. As participants explained, offering remediation was challenging when it was necessary to engage in 'much more of an intensive process than just attending a CPD conference on anything' (CPD 3). 
Interestingly, when our participants spoke of remediation in this way, they concluded that the logical organisation to provide remediation support would be a university. They suggested that the only reasons why a university might not offer this support would refer to logistical issues such as lack of resources. Echoing the conclusions of many participants, one university postgraduate medical education (PGME) dean stated:

From a capability - capacity is what I'm talking about - from a knowledge point of view, like, the universities are, I think, the best equipped to do this kind of work. It's just a capacity issue. No-one has the time and the effort to put [in] to it. (PGME Dean 1)

\section{Remediation as a regulatory process}

In addition to this conceptualisation of remediation as one end of a seamless educational continuum of increasing support, participants also expressed a second perspective, one that entailed a profound shift in the nature and meaning of remediation once the term was invoked. In this second conceptualisation, participants acknowledged that remediation is the equivalent of imposing upon - or even revoking - an individual's selfregulatory process. Here, the defining characteristic of remediation is the individual's loss of choice. Thus, 'remediation is essentially directed education. So it's education that is not recommended, but is education that's required' (RA 3). It is 'a formalised process where the physician is - where that process is supervised and monitored in a way by the regulatory authority' (RA 3). The difference between remediation as an educational continuum and remediation as a regulatory process was explicitly articulated by a CPD dean:

... inherent in quality improvement is that notion of self-audit and it doesn't force you to make change. [In the] remediation process, by definition, you need to make change if you're going to continue. (CPD 1)

This removal of choice impacts all aspects of the learning process. It not only mandates what the individual needs to learn and how much he or she needs to learn, but it also mandates 'focused follow-up ... to ensure that it did happen'. (RA 3) Although there may be efforts to mask or soften the blow of this removal of autonomy, ultimately, the loss of educational autonomy is complete:

I think very few do it on their own, seek it out. I think most of them [i.e. physicians who need remediation] are eventually brought into a position of complicity, usually through the efforts of their chief or their chief medical officer. Maybe with some peers. I would say probably twothirds to three-quarters of them eventually do something voluntarily that they've come to be convinced is going to be in their best interest. But there's always a subset - and I would say it's probably $25 \%$ or less - who really have to have it forced on them, either through threat of losing their privileges or through a complaint to the college or something of that nature. (Department head [DH] 1)

Not surprisingly, in this second conceptualisation, remediation is stigmatised. It is not a process in which every physician engages constantly; it is for a select few. It is 'the term for those guys that are actually really bad and must get better to retain the rights of, and the privileges of practice' (CPD 5). In this conceptualisation, remediation denotes, 'obviously 
somebody who is in trouble' (CC 1). Surprisingly, in this second conceptualisation, the point at which an individual requires remediation is also somewhat arbitrary:

There's not ... something like a checklist saying "if you have these things checked off then you have to go to remediation"; it's really more an expert opinion/consensus kind of process. (RA 1)

When the defining characteristic of remediation was seen to be the removal of educational autonomy, the responsibility for remediation was seen as belonging to the organisation responsible for professional autonomy (i.e. the regulatory authority). Universities could 'provide a service' as several university-based interviewees mentioned, but only if participants 'realised we weren't forcing them, that someone else was forcing them to take our programme' (CPD 2). University offices, it appears, did not wish to be viewed as complicit in removing an individual's autonomy.

\section{Simultaneous conceptualisations}

These two differing conceptualisations of remediation were not mutually exclusive. They were held simultaneously, to greater or lesser degrees, within similar stakeholder organisations and frequently by the same individuals. Slippage and confusion between the two simultaneously held conceptualisations were epitomised by participants' ubiquitous references to a 'bell curve', referring to a normal distribution of physicians' performance. When invoking this reference, participants talked about variation in learning and improvement needs, with different degrees of remediation along the curve. The bell curve references the first model, remediation as an educational continuum, with those at the lower end of the curve simply needing progressively higher levels of support because of progressively increasing performance gaps. However, participants also appeared to use the lowest portion of the curve to reference a qualitatively different group, referring to the population at the tail end of the curve as a distinct group:

... a select group, you know, who are way outside of the curve, maybe 1\% or 2-3\% of the numbers I hear, you know, of physicians that I would look at, that are really in trouble. (CPD 3)

... the bottom tail, that $2.5 \%$ or less that are struggling to sustain, you know, the quality of care throughout their whole professional lives. (CC 2)

I think it's probably in that sort of 5\%. It's not a big number. I think it's sort of your bell curve kind of thing that it's probably the tailing $5 \%$. (RA 4)

Although participants did not always knowingly or explicitly distinguish between the two conceptualisations, they did notice and identify some of the consequences of failing to do so. For example, participants recognised that failure to distinguish between the concept of remediation as an educational continuum and the concept of remediation as a regulatory process could stigmatise remediation processes, as well as other points along the continuum of educational supports:

I don't believe that currently we have a general professional sense of what quality improvement is. It seems to me from my dealings with this in education circles is that physicians think that, 
you know, quality improvement, quality assurance means that someone doesn't think you're doing a good job. And that if you have to get involved with quality improvement, it's not quite as bad as remediation. But something's not quite right with your practice and therefore you have to improve the quality a little bit ... And it's a certain sense of entitlement that l've become a doctor now and so I know what's best and therefore, you know, don't question me ... So when you talk about remediation in that context, then it's more negative than just trying to improve what you're doing. (Health authority [HA] 1)

... docs right now don't trust the [performance improvement] process. So they kind of just try to get through it and nothing good ever happens. But they don't engage with it in a way of looking at it - here's an opportunity I can improve. (CPD 3)

Hence, although regulatory authorities might develop physician performance improvement initiatives as a means of ensuring competent care across the profession, practising physicians equate this process with 'remediation lite' and view it as threatening.

\section{Discussion}

The aim of this study was to understand how remediation is conceptualised by those in organisations that have a stake in the process. Our findings suggest that most of these individual stakeholders simultaneously hold two differing conceptualisations of remediation: remediation as an educational process on a continuum of learning, and remediation as a regulatory process. That is, all interviewees described remediation as part of an educational continuum and most also viewed it - explicitly or implicitly - as a regulatory process. Interviewees moved between the two conceptualisations but did not always appear aware that they were doing so. Importantly, the two framings have different implications for the degree to which remediation can be easily incorporated into the professional processes of maintaining competence.

Our participants' propensity to conceptualise remediation as an educational process and part of the educational support continuum mirrors the emphasis seen in the literature and appears to be consistent across (at least Western) health care systems. ${ }^{6,7,24-28}$ For example, in the UK, where doctors are employees, the National Clinical Assessment Service framework suggests that remediation 'should still be seen as part of an educational continuum for improving practice.26 Also consistent with the literature, most of our interviewees did not formally reference education theories although they frequently indirectly referenced adult learning principles such as helping individuals identify their own learning needs. This suggests that the remediation community may need to become more conscious of which underlying educational theories underpin remediation programmes and to consider whether alternative theories, whether educational or, more broadly, change theories, might prove more helpful in the remediation of practising physicians.

However, even this shift in emphasis from learning to practice change may be insufficient to redress the underlying difficulties in invoking remediation because it fails to address 
the sociological implications embedded in the regulatory aspect of the process. The literature is fairly silent about the implications of remediation as a regulatory process and the associated removal of autonomy. The grey literature from the UK, where remediation is viewed in many cases as performance management, mentions 'clinical governance', but the principles are all educational. This silence regarding the regulatory aspect of remediation may simply derive from the fact that it appears obvious, but the underlying implications may not be fully appreciated. ${ }^{19,29}$ As sociologists consider autonomy to be the defining characteristic of a professional, we would argue that the invocation of remediation may be considered at least a temporary de-professionalisation. ${ }^{15}$ Importantly, this removal of professional autonomy and the resultant de-professionalisation do not seem to be intended as overt punishment. In fact, we interpret our participants' emphasis on the continuum of support, at least in part, as an effort to avoid a framing of remediation as punishment. Rather, the intended goal of remediation is to support the practitioner's return to competent practice. The resulting de-professionalisation therefore might be considered as an unintended consequence of invoking the regulatory process of remediation. Even if not intended as punishment, the implications of this aspect of the remediation process may be profound.

Educational activities are generally not associated with stigma; as our participants noted, everyone has gaps, and everyone can improve. The stigma lies in the loss of autonomy, in no longer being in control of one's continuing education or one's practice, and in the perception that one has lost an important component of one's professional identity. Therefore, restructuring remediation to enable the retention of autonomy to the fullest possible extent might soften the stigma and increase the willingness of physician organisations not traditionally involved in remediation to support the process in various ways.

\section{Limitations}

This study was conducted in the Canadian context at a particular point in time and we acknowledge that findings might differ in different contexts. For example, Canadian physicians are generally independent contractors rather than employees of the state, such as they are in the UK where remediation appears to be viewed as performance management. Conversely, Canadian culture is not as fiercely individualistic as American culture, which has seen several states enact laws that forbid mandatory CME programmes that aim to measure core competencies in medicine. ${ }^{30}$ Given the high variability in the provincial practices that inform our research, we posit that our findings may transfer to other contexts, but further research is needed to determine the extent to which this is in fact the case.

Additionally, for this study, we specifically targeted those who had an administrative stake in the process of remediating physicians in practice. As such our participants may not be representative of the broader practice community. We suspect that, relative to the physician 'in the trenches', they are likely to be more supportive of performance improvement initiatives and the resultant identified need for supported practice change, but we currently have no data to verify this. Further, we cannot say to what extent our findings will apply to other points in the education process, or whether, for example, the 
loss of autonomy is as much a concern in the postgraduate or undergraduate education realms. Perhaps most importantly, we do not know whether and how those engaged in the process of remediation (preceptors and remediatees) are influenced by these conceptualisations during the actual activity of remediation.

\section{Conclusions}

Remediation stakeholders simultaneously view remediation as one end of a continuum of educational support and as the removal of educational autonomy, or as a de facto de-professionalisation of the individual involved. Understanding that stakeholders frequently approach the complex issue of remediation with two different perspectives without conscious awareness of doing so may help to resolve several challenges in the field, including the questions of what falls under the umbrella of remediation and who should be responsible for offering remediation support. Our findings suggest the need for conceptual clarity around remediation both to ensure that we illuminate logistical dilemmas in enacting remediation and to address the stigma of 'de-professionalisation' that even minor educational supports (such as feedback) might invoke by association. Until we acknowledge and address the dichotomy of remediation as both educational and regulatory, the profession will continue to struggle with logistics, and organisations focused on supporting physicians, such as medical associations and CPD offices, will hesitate to become involved, to the detriment of the public, the individuals requiring remediation and the profession as a whole. 


\section{REFERENCES}

1. Archer J, de Bere SR. The United Kingdom's experience with and future plans for revalidation. Journal of Continuing Education in the Health Professions 2013;33 (suppl 1):S48-53

2. Sargeant J, Bruce D, Campbell CM. Practicing physicians' needs for assessment and feedback as part of professional development. Journal of Continuing Education in the Health Professions 2013;33 (Suppl1):S54-62.

3. Jacques A. Physician Practice Improvement. Ottawa, Ontario: Federation of Medical Regulatory Authorities of Canada 2016.

4. Allsop J, Jones K. Protecting patients: international trends in medical governance. In: E Kuhlmann, M Saks, eds. Rethinking Professional Governance: International Directions in Health Care. Bristol: Policy Press, University of Bristol 2008; 15-28.

5. Goulet F, Gagnon R, Gingras ME. Influence of remedial professional development programs for poorly performing physicians. Journal of Continuing Education in the Health Professions 2007;27 (1):42-48.

6. Norton PG, Ginsburg LS, Dunn E, Beckett R, Faulkner D. Educational interventions to improve practice of nonspecialty physicians who are identified in need by peer review. Journal of Continuing Education in the Health Professions 2004;24 (4):244-252.

7. Rhydderch $M$, Matthews $P$, Beech $M$. The advanced training practice network: providing prescribed further training for general practitioners in Wales. Education for Primary Care 2007;18 (5):572-581.

8. Price T, Archer J. UK policy on doctor remediation: trajectories and challenges. Journal of Continuing Education in the Health Professions 2017;37 (3):207-211.

9. Humphrey C. Assessment and remediation for physicians with suspected performance problems: an international survey. Journal of Continuing Education in the Health Professions 2010;30 (1):26-36.

10. Varpio L, Aschenbrener C, Bates J. Tackling wicked problems: how theories of agency can provide new insights. Medical Education 2017;51 (4):353-365.

11. Cleland J, Leggett H, Sandars J, Costa MJ, Patel R, Moffat M. The remediation challenge: theoretical and methodological insights from a systematic review. Medical Education 2013;47 (3):242-251.

12. Bleakley A, Bligh J, Browne J. Medical Education for the Future: Identity, Power and Location. London: Springer 2011.

13. Prescott-Clements L. Rethinking remediation: a model to support the detailed diagnosis of clinicians' performance problems and the development of effective remediation plans. Journal of Continuing Education in the Health Professions 2017;37 (4):245-254.

14. Bourgeois-Law G, Teunissen PW, Regehr G. Remediation in practicing physicians: current and alternative conceptualizations. Academic Medicine 2018;93 (11):1638-1644

15. Friedson E. The characteristics of a profession. In: SL Earle, G Letherby, eds. The Sociology of Health Care: A Reader for Health Professionals. Basingstoke: Palgrave Macmillan 2008; chapter 23, pages 247-257.

16. Armstrong D. Clinical autonomy, individual and collective: the problem of changing doctors' behaviour. Social Science\& Med 2002;55 (10):1771- 1777.

17. Barber B. Control and responsibility in the powerful professions. Political Science Quarterly 1978;93 (4):599-615. 
18. Tezuka K. Physicians and professional autonomy. Japanese Medical Association Journal 2014;57 (3):154-158.

19. Somerset Partnership, NHS Foundation Trust. Remediation, Re-skilling and Rehabilitation Policy for Medical Staff. London: National Clinical Assessment Service 2012.

20. Cohen D, Rhydderch M, Cooper I. Managing remediation. In: T Swanwick, ed. Understanding Medical Education: Evidence, Theory, and Practice. Edinburgh: Association for the Study of Medical Education 2010:366-378.

21. Hauer KE, Ciccone A, Henzel TR, Katsufrakis P, Miller SH, Norcross WA, Papadakis MA, Irby DM. Remediation of the deficiencies of physicians across the continuum from medical school to practice: a thematic review of the literature. Academic Medicine 2009;84 (12):1822- 32.

22. Charmaz K. Constructing Grounded Theory, 2nd edition London: Sage Publications 2014.

23. Glaser BG, Strauss AL. The Discovery of Grounded Theory: Strategies for Qualitative Research. Chicago, IL: Aldine 1967.

24. Lillis S, Takai N, Francis S. Long-term outcomes of a remedial education program for doctors with clinical performance deficits. Journal of Continuing Education in the Health Professions 2014;34 (2) 96-101.

25. Williams BW. The prevalence and special educational requirements of dyscompetent physicians. Journal of Continuing Education in the Health Professions 2006;26 (3):173-191.

26. National Clinical Assessment Service. The Back on Track Framework for Further Training. London: NCAS 2010.

27. Bower E, English C, Choi D, Cedfeldt A, Girard D. Education to return nonpracticing physicians to clinical activity: a case study in physician reentry. Journal of Continuing Education in the Health Professions 2010;30 (2):89-94.

28. Cerda JJ, Van Susteren TJ, Hatch R, Herkov M. Remedial education: can this doctor be saved? Transactions of the American Clinical and Climatological Association 2000; 111:188-195; discussion 196-197.

29. Smith R. Remediation Working Group Report. London: Academy of Medical Royal Colleges 2012.

30. Edison M. Oklahoma bans forced MOC, becomes the first "Right to Care" state. Rebel MD 2018 https://d4pcfoundation.org/oklahoma-bans-forced-moc-becomes-the-first-right-to-carestate/ [Accessed 11 July 2018.] 


\section{Appendix A}

\section{Semi-Structured Interview Questions}

- Please describe for me what picture comes to mind when you think of a "typical" physician whose practice does not meet the expected standard in one or more ways.

- When you think of how one might bring that physician's practice back up to an acceptable standard, what methods do you think would be most successful and what in your experience or reading suggests that to you?

- "Remediation" is a term that is often used for the process of bringing an under functioning physician's practice up to an acceptable standard. What does that term evoke for you?

- Since "remediation" is currently the most commonly used term, that is the term I will use for the remainder of this interview. Please tell me about your experience, both from an organizational and personal perspective, with the remediation of practicing physicians.

- In a perfect world where the grass was always green and money was never an issue, how would remediation for practicing physicians be addressed?

- When you think of all the issues facing the medical profession and the health care system today, where does the issue of physician remediation fit in? 



\section{Attitudes Towards Physicians Requiring Remediation: One-of-Us or Not-Like-Us?}

Gisèle Bourgeois-Law | Pim W Teunissen | Lara Varpio | Glenn Regehr 


\section{ABSTRACT}

Purpose The data for this paper were collected as part of a larger project exploring how the medical profession conceptualizes the task of supporting physicians struggling with clinical competency issues. In this paper, the authors focus on a topic that has been absent in the literature thus far-how physicians requiring remediation are perceived by those responsible for organizing remediation and by their peers in general.

Method Using a constructivist grounded theory approach, the authors conducted semistructured interviews with 17 remediation stakeholders across Canada. Given that in Canada health is a provincial responsibility, the authors purposively sampled stakeholders from across provincial and language borders and across the full range of organizations that could be considered as participating in the remediation of practicing physicians.

Results Interviewees expressed mixed, sometimes contradictory, emotions toward and perceptions of physicians requiring remediation. They also noted that their colleagues, including physicians in training, were not always sympathetic to their struggling peers.

Conclusions The medical profession's attitude toward those who struggle with clinical competency - as individuals and as a whole - is ambivalent at best. This ambivalence grows out of psychological and cultural factors and may be an undiscussed factor in the profession's struggle to deal adequately with underperforming members. To contend with the challenge of remediating practicing physicians, the profession needs to address this ambivalence and its underlying causes. 
Remediation processes in clinical practice can vary significantly across jurisdictions in terms of organizational responsibility, types of experiences organized, and outcome measures. In contrast, the educational conceptualization of the remediation activity itself has remained relatively constant. Recent papers have suggested that understanding the remediation of practicing physicians beyond its purely educational aspects could provide insights that lead to better remediation practices. ${ }^{1,2}$ For example, framing the goal of remediation not as a narrow educational issue (i.e. requiring improved knowledge or skills) but rather as a behavioral change process (i.e. exploring what is required to engender lasting practice improvement for this person in this situation) might address the personal barriers to a successful outcome. ${ }^{1}$ Additionally, acknowledging that remediation involves both educational and regulatory aspects might lead to the development of strategies that address its stigma and improve its acceptability. ${ }^{3}$ While there is increasing emphasis on the process itself, one focus that remains absent from the discussion is an explicit articulation of how physicians requiring remediation are perceived both by those responsible for organizing remediation and by their peers in general. Understanding how the profession perceives its members who require remediation could enable us to address remediation in new ways.

Currently, the literature only obliquely references how physicians requiring remediation are viewed by their peers or the profession as a whole. For instance, program descriptions imply that physicians requiring remediation need more support than their peers. ${ }^{4-7}$ In other work, personal and practice characteristics have been identified as factors increasing the likelihood of requiring remediation. ${ }^{8-11}$ Across these manuscripts, references to those needing remediation are generally dispassionate, avoiding both empathetic and judgmental language. At the same time, there are hints that the situation may be more complex, with these individuals sometimes framed as a minority of 'bad apples.'12-14 Anecdotal evidence suggests that physicians tend to distance themselves from struggling colleagues ${ }^{15}$ and that physicians who don't 'fit in' in various ways, including by the quality of their performance, are asked to leave their practice community. ${ }^{16(P .62)}$ These tendencies might make it more difficult for struggling physicians to admit needing help or accessing remediation.

In this study, we wished to go beyond implicit and anecdotal representations and develop a better understanding of how physicians requiring remediation are viewed by the medical community. To develop this understanding, we talked with members of organizations that have a stake and/or role in the remediation of practicing physicians. Our research question asked, "How do remediation stakeholders conceptualize physicians requiring remediation for clinical competence issues?"

\section{Method}

The data for this paper were collected as part of a larger project whose purpose was to understand how the Canadian medical profession conceptualizes the support of physicians struggling with clinical competence issues. Remediation is a process that varies greatly across jurisdictions. Given that in Canada health is a provincial responsibility, we 
interviewed stakeholders from the full range of organizations, across provincial and language borders, that might be considered as having some part in the remediation of practicing physicians. While the data are Canadian-centered, we suggest that the breadth of contexts included will provide insights that are transferable to other jurisdictions and provide an example of the methods that can be used to replicate findings elsewhere. ${ }^{17}$ We focused on remediation of physicians for clinical competence (e.g. knowledge, skills, attitudes) rather than for rehabilitation for impairment due to substance abuse or illness, or sanctions for grossly unprofessional behavior.

In a previous paper from this program of research, ${ }^{3}$ we described how Canadian remediation stakeholders simultaneously held 2 conceptualizations of the remediation process: The first foregrounds remediation as an educational process, and the second foregrounds the regulatory aspect and the individual's loss of choice. In that work, we noted how the tendency to focus on one or the other of these conceptualizations, rather than grappling with both simultaneously, contributed to some of the confusion and challenges surrounding remediation processes. In this paper, we shift our focus away from the processes of remediation to attend to the individuals receiving remediation. Specifically, we concentrate on the attitudes and emotions that stakeholders demonstrated toward those who required remediation.

G.B.-L. conducted 1-hour semistructured interviews between April 2016 and June 2017 with 17 remediation stakeholders across Canada. These included 4 registrars or senior representatives of the provincial regulatory authorities, 3 postgraduate and 4 continuing professional development associate deans of Canadian medical schools, 1 representative from each of the 2 national certifying colleges, 2 health authority department heads, 1 health authority medical staff support director, 1 medical faculty dean, and 1 former director of a physician health program (the total is $>17$ because some participants fit into more than 1 category). Sample size was informed by accepted standards for sample size in qualitative research. ${ }^{18}$ In keeping with those standards, interviews continued until we achieved 2 aims: (1) We had collected sufficient data to develop new knowledge around the study phenomenon, and (2) interviews were no longer revealing significantly novel insights. Interview questions were deliberately kept quite broad in scope-for example, "Please tell me about your experience, both from an organizational and personal perspective, with the remediation of practicing physicians" and "Please describe for me what picture comes to mind when you think of a 'typical' physician whose practice does not meet the expected standard in one or more ways." We sought to understand how stakeholders conceptualize remediation and so did not restrict the parameters of the questions to avoid leading the conversation in any particular direction.

Interviews were audio recorded and anonymized during transcription by an external transcription service. To preserve anonymity, participants are referred to by number in the manuscript (e.g. P\#). Interviews were conducted in either of Canada's 2 official languagesEnglish and French. All data were translated into English for analysis. To ensure accuracy of the translation of the French interviews, these transcripts were translated into English by 1 professional translator, then translated back into French by a different professional translator-a process called back translation-after which G.B.-L. compared the 2 French 
documents to ensure that the translations correctly captured the participants' meanings. The professional translators addressed any concerns regarding perceived meanings until the English transcripts accurately reflected francophone participants' expressions.

Through the use of a constructivist grounded theory approach, interviews were analyzed iteratively such that data analysis occurred apace with data collection. ${ }^{20}$ The initial interview protocol is available elsewhere, however, consistent with constructivist grounded theory methodology, insights from initial interviews informed a revision of the protocol for subsequent interviews. ${ }^{3}$ G.B.-L. coded all interviews, L.V. reviewed the codes, and the entire research team discussed the coding structure on multiple occasions to construct the theoretical framework. Given the constructivist orientation of this research, it is important for us to reflect on our perceptions and experiences of the phenomenon in question. G.B.-L. is a retired physician who spent 10 years as the director of a clinician assessment and remediation program. These experiences enriched her understanding of the data, but vigilance was required to avoid preconceptions unduly influencing the interpretation of the results. P.W.T. is a gynecologist with a PhD in workplace learning in health care and 10+ years of experience in both qualitative and quantitative research in the field of health professions education. L.V. is a qualitative research scientist who has studied health professions education for $10+$ years. L.V. has not previously studied the remediation of practicing physicians, nor does she know physicians who required remediation (that she is aware of). G.R. is a $\mathrm{PhD}$ trained researcher in cognitive science with 25 years of experience conducting and supporting both quantitative and qualitative scholarships in the field of health professions education.

The study received ethical approval from the UBC Behavioral Research Ethics Board (protocol no. H16-00529).

\section{Results}

Our participants acknowledged that remediation is, as 1 participant put it, "emotionally and cognitively difficult" (P4) and agreed that, most often, "it's the candidate that's the hot potato, not the process" (P3). Our interviewees expressed mixed, sometimes contradictory, emotions toward and perceptions of physicians who require remediation. They observed that their colleagues, including physicians at various levels of training, were not always sympathetic to their struggling peers. The perceptions and emotions associated with remediatees were described in several different ways.

\section{Physicians requiring remediation are one-of-us}

Respondents often saw physicians requiring remediation as unfortunate members of the community, not that different from other physicians-"anyone can run into trouble for any number of reasons" (P2) - or even from themselves: "there but for the grace of God go I" (participant commenting that they could also find themselves in similar circumstances) (P8). 
Several respondents spoke of physicians drifting into incompetence or falling into incompetence. When respondents were describing incompetence in this way, it was not framed as a characteristic or trait of the physician. Rather, incompetence became a hopefully temporary state one arrived at through inattention and/or external circumstances, a place that any physician could find themselves in through carelessness or bad luck:

\section{It happened insidiously, gradually; they didn't notice. (P3)}

I think we're all probably at risk of that. They just happen to have fallen victim to it at a certain part of their life. (P4)

When physicians struggling with competence issues were framed as one-of-us, helping struggling colleagues was described as a professional responsibility to patients/public - "It's a collective responsibility to make sure that quality care is delivered and we get better outcomes" (P12) - and as an obligation to the struggling colleagues: "It's not right if we can call someone out and pull them out of practice but actually don't provide any resources for them. So I think there's a moral imperative" (P2).

\section{Physicians who require remediation are not-like-us}

Participants also viewed physicians who were struggling with competence issues as not-like-us-that is, no longer one-of-us. Participants described physicians requiring remediation as no longer being a fully functioning member of the physician community: "So if you don't meet the mark, I guess you can no longer be part of this group" (P5). Struggling physicians were designated as somehow different from their peers: "Maybe you just don't have it in you to be what you need to be" (P6). They might not even be conceived of as normal people because "normal people with a reasonable degree of insight might possibly respond to circumstances differently" (P5). In contrast to the previous conception of wrestling with competence as a state that the struggling physician unintentionally drifted to, this perspective appeared to conceive of incompetence as resulting from a trait or characteristic of the person: "You didn't have the discipline or the insight to recognize that you're beginning to struggle. Or if you did you didn't have the motivation to do anything about it" (P15).

When struggling physicians were framed as not-like-us, participants recognized less of a social, professional, or personal imperative to support remediation:

It comes down to, well, do you keep on supporting someone if they want to keep on practicing and they're maybe losing their edge. And that they've made an individual decision to work in isolation and therefore, not potentially participate in any community practice that would be able to help them. So my concern always is so, yeah, you put in-have a remediation program and then-but what happens after that? (P6)

When considering struggling physicians as not-like-us, participants also expressed doubts as to remediation's long-term success: 
They'll do it to keep us happy, they'll successfully complete the training period, they'll focus on the patient during the training period - (but then they) go back to their settings, it doesn't make any difference. (P3)

\section{Ambivalence}

Respondents did not neatly divide into 2 groups in their attitudes toward physicians requiring remediation. Struggling physicians were not categorized as either oneof-us or not-like-us. Rather, our research participants often shifted between these conceptualizations. This duality existed not only between participants but also within individual participants. The internal struggle to simultaneously maintain conflicting attitudes of this-could-happen-to-me while at the same time othering those requiring remediation was clearly evident:

Are we not at risk of being prey or falling into the same sort of traps that these people have fallen into? And yet at the same time we should not forget that the vast majority of physicians seem to manage, seem to do a pretty good job. Seem not to find themselves in those circumstances. (P5)

Even where respondents were positioning themselves as sympathetic to struggling colleagues, othering language crept into their expressions - for example, "a bit of a tainted product" (P7).

Our respondents also described such ambivalent attitudes toward struggling colleagues as existing in the broader physician community. On the one hand, participants noted sympathetic attitudes that physicians are frequently "extremely cautious about hurting their colleagues" (P11). The one-of-us/could-be-me attitude in the larger community was described by 1 participant who stated:

People often don't feel that they're in a position to sort of call someone else out on it. I think a lot of people feel like, well, you know, I'm not perfect either, so I've got to be careful before I throw stones. Because, you know, someone could turn around and say that I'm less than perfect, which is certainly true. (P13)

On the other hand, participants also noted negative responses:

The physician community is, you know, "circle the wagons and keep them out" lots of times. There's not a lot of support and it's not helpful to that person.... we still have that outer veneer, you know, we're Teflon... and if you don't meet [the bar] you're bad. (P1)

In referencing the negative responses, participants suggested that these approaches started as early as medical school:

I think we're brutal. Frankly, I see it even in medical students and residents. If you start showing any signs of struggle or weakness the first instinct of many physicians is just to want you to be gone... I think as a guild physicians are very hard on each other. (P16) 
It was unclear how and when participants resolved this ambivalence and decided whether remediatees in general or particular individuals are one-of-us or not-like-us. However, associated comments suggest possible factors that might come into play. One such factor was the problem that instigated the need for remediation and its duration:

For willing physicians who are-don't have any professionalism or behavioral concerns and need some help getting back on track, I think that they should at least be given a chance. (P8)

I suspect that someone who's left practice for family or health reasons has a bit of a different latitude with this than someone who's just been struggling for a while and then finally crumbles, or has a major issue. (P6)

Another potential factor in resolving the ambivalence was the physician's response to the identified practice gap, a response that appears to be linked to insight:

If one accepted the fact that their care wasn't standard, that'd be the more important thing, I think, that they actually accepted it and did whatever they needed to overcome it. (P10)

As long as there's some insight and they're at a proper stage of kind of, you know, maybe that grieving process or acceptance of something's not right. (P7)

However, this position, too, was laced with ambivalence. Participants frequently referenced insight-not only its importance in determining the individual's response to being told they had a gap but also its role in leading to the need for remediation and the degree of engagement with remediation. However, participants also acknowledged that medical culture doesn't always encourage the development or expression of such insight:

This insight isn't allowed. I think many people have it internally, but they can't express it or they're forced to conceal it. (P16)

You end up with a bit of-almost feeling like you have to have this, you know, all-knowing persona which none of us have and none of us could possibly have. And so it's hard to admit that no, you know, I-my skills aren't where they need to be. I'm weak on this area. I'm weak on that area. You're not expected to be weak. You're expected to be in control and so I think it sets up a fair degree of stress. And, I mean, you know, you've been told you're a bright person and stuff. You don't want to admit that you're not or that you're anything less than what people expect of you or think of you. (P13)

\section{Discussion}

Our data suggest that ambivalence regarding struggling physicians may be an underrepresented contributor to the profession's challenges in addressing remediation. Some remediatees are acknowledged as one-of-us who, for a variety of reasons not always under their control, fell into trouble; yet, results show that stakeholders 
also other struggling physicians. This othering has several implications for both struggling physicians and remediation programs.

\section{Othering of struggling physicians}

The psychology literature suggests several reasons why physicians might other their struggling colleagues. The tendency to other may have been influenced by whether our participants believed that competence issues are a temporary state or a trait (i.e. whether someone had drifted into incompetence due to circumstances vs not having it in them to practice competently throughout the practice life cycle). As part of this consideration, the fundamental attribution error might be at play, and if so, we might anticipate a discrepancy in attributions, with remediatees tending to view their problems as due to circumstances (a rationale which others label lack of insight) while their peers would tend to regard these same problems as arising because of innate characteristics. ${ }^{21}$ In this sense, our participants may have held implicit bias regarding struggling physicians and their ability to improve. ${ }^{22}$ For example, while older physicians do have a lower success rate (defined as meeting educational objectives), starting with the assumption that an individual physician may not be able to return to safe practice because of age or other characteristics would be considered implicit bias. ${ }^{5}$

The othering of struggling physicians may also arise secondary to their perceived failure to enact the rights and responsibilities of professional (clinical and educational) autonomy. These individuals have not self-regulated, either internally or with external help, to a sufficient degree to identify areas where their practice needed improvement and/or they have not sought out the necessary continuing education or practice supports to maintain competence. By virtue of this failure, they suffer what might be considered a de facto deprofessionalization and therefore are no longer one-of-us. ${ }^{3}$ The invocation of remediation might be considered a signal that they are no longer fully legitimate participants in the community of practice.

However, it is also possible that there are additional reasons why physicians might wish to consider colleagues who require remediation as not one-of-us, to exclude or other them. As Coupland notes, "social categorization is never neutral. We invoke grouplevel categories for specific rhetorical and social purposes." ${ }^{\prime 23}$ Thus, on a collective level, the profession may resent struggling members because they threaten the profession>s reputation and its ensuing professional autonomy to self-regulate. On an individual level, recent research reveals that physicians are generally insecure and that even very successful doctors may have characteristics of imposter syndrome. ${ }^{24}$ Perhaps struggling physicians provoke discomfort and serve as a painful reminder that any physician could be in a similar situation some day. Framing those who have to undergo remediation as deserving it, or as somehow different from other physicians, may perhaps assuage the anxiety about possibly ending up in a similar situation..$^{25}$ Thus, ironically, the could-be-me framing of a struggling physician's circumstance might lead less to a one-of-us designation and instead produce a reactive response of self-protective othering. 


\section{Othering and insight}

Our data suggest that the tendency to other struggling colleagues is influenced by both the circumstances that triggered the need for remediation and the response to that need. The latter is influenced in turn by the level of insight the struggling practitioner possesses or develops as a result of feedback. Our respondents frequently referenced insight. Apart from logistical issues regarding accessing remediation support, insight was deemed the biggest challenge to successful remediation. Insight is so important to a practicing professional that it is included in many organizational constructions of the physician. For example, in Canada, the CanMEDS 2015 document identifies insight as a "Key Concept" under the role of "Professional," and the CanMEDS-Family Medicine framework includes as a Professional competency to "strive to heighten personal and professional awareness and insight." ${ }^{26,27}$ Lack of insight is seen as compounding any clinical competence deficiency and can elevate it to a professionalism issue. Professionals are supposed to have insight into their practice and learning needs and are expected to find ways of identifying their gaps. If a professional has insight into their gaps, then they don't need remediation; instead, they need resources to help them improve their practice. They are still a professional, still one-ofus. Physicians who lack insight into the fact that they have significant practice gaps are not demonstrating an important characteristic we expect of a professional; they are no longer one of us. The problem, as our respondents intuited, is that while we demand insight, the culture doesn't encourage its expression unless and until one is "in trouble." Until then, practicing physicians are expected to maintain what one respondent labeled a "Teflon" attitude - the outer veneer of perfection, which fosters finding external justifications for less-than-optimal outcomes. Nor does the culture encourage demonstrating vulnerability; while learners are expected to "exhibit self-awareness," demonstrating vulnerability can be hazardous for practicing clinicians. ${ }^{26,27}$ As previously noted by another respondent, "If you start showing any signs of struggle or weakness, the first instinct of many physicians is just to want you to be gone." Demonstrating vulnerability can also affect credibility for preceptors, which discourages role modeling by faculty. ${ }^{28}$

\section{Implications}

It appears that psychological, cultural, and logistical factors may be interacting to form a vicious circle with regard to remediation in practicing physicians and that the tendency to other struggling physicians may be an important component of that circle. Struggling physicians, wishing to avoid the pain of being othered, avoid admitting significant gaps to self and to community members until these are advanced to the point that remediation will be complicated and long-term successful return to practice less likely. This in turn leads to a decreased willingness among stakeholders and the profession to provide the necessary support and resources that would contribute to successfully addressing what have become significant gaps. We are not suggesting that addressing the tendency to other struggling physicians will magically disrupt the circle. Almost certainly there is a complex and interacting set of system factors that need to be addressed, so simple causeeffect constructions of a solution will be insufficient. For example, it could as easily be argued that increasing remediation resources would not only contribute toward earlier referral and positive outcomes but also might serve to normalize the need for help in addressing gaps and thus decrease the tendency to other. We would assert, however, that 
it will be difficult to disrupt the circle without addressing the profession's ambivalence toward its struggling members.

Since, as our participants suggested, the ambivalence and othering of struggling colleagues starts early, we should start addressing it early. It would send a powerful message if, on the first day of medical school, the dean stated that the practice of medicine is difficult and that it is inevitable that some will struggle for a variety of reasons unrelated to intelligence or personal worthiness. If one or more senior faculty members then related stories of their own struggles in training and stressed the existence of nonjudgmental support programs, perhaps some of the associated stigma could be diminished. A similar message could be repeated by program directors at the beginning of residency. The best way to address our collective ambivalence toward remediation may be to normalize the need for support and to ensure the availability of that support. While it may be naive to presume that everyone needing support will have the insight to avail themselves of it, we may hope to slowly change the culture so that gaps are acknowledged and addressed before they cause harm or grow to the extent that they require expensive, extensive retraining.

Our methodological decisions in this study, of course, do place some limitations on our conclusions and implications. For example, we interacted only with remediation stakeholders in this initial study. It is possible that physicians in practice with no connection to a university or to a regulatory authority would have different attitudes, although comments from our participants regarding attitudes they perceive in the broader community suggest that this is unlikely to be the case. Further, in an effort to avoid prejudicing responses, our questions were open-ended without specifically asking participants about their views of physicians who required remediation. However, it is possible that asking such specific questions could have led to slightly different results or enabled further exploration of underlying conceptions. These limitations suggest that further research is needed to ensure that remediation is being addressed on the basis of well-grounded, evidence-based theoretical constructs.

\section{Conclusions}

The medical profession's attitudes toward those of its members who struggle with clinical competence issues are ambivalent at best, both in the profession as a whole and in individual members. This ambivalence results from a combination of psychological and cultural factors and may be an undiscussed factor in the profession's struggles to deal adequately with underperforming members. The profession needs to address this ambivalence and its underlying causes, perhaps starting as early as medical school, if it is to confront the challenge of remediation in practicing physicians. 


\section{References}

1. Prescott-Clements L, Voller V, Bell M, Nestors N, van der Vleuten CPM. Rethinking remediation: A model to support the detailed diagnosis of clinicians' performance problems and the development of effective remediation plans. The Journal of Continuing Education in the Health Professions 2017;37 (4):245-254.

2. Bourgeois-Law G, Teunissen PW, Regehr G. Remediation in practicing physicians: Current and alternative conceptualizations. Academic Medicine 2018;93 (11):1638-1644.

3. Bourgeois-Law G, Varpio L, Regehr G, Teunissen PW. Education or regulation? Exploring our underlying conceptualisations of remediation for practising physicians. Medical Education 2019;53 (3):276-284.

4. Rhydderch $M$, Matthews $P$, Beech $M$. The advanced training practice network: Providing prescribed further training for general practitioners in Wales. Education for Primary Care 2007;18 (5):572-581.

5. Norton PG, Ginsburg LS, Dunn E, Beckett R, Faulkner D. Educational interventions to improve practice of nonspecialty physicians who are identified in need by peer review. The Journal of Continuing Education in the Health Professions 2004;24 (4):244-252.

6. Miller F, Jacques A, Brailovsky C, Sindon A, Bordage $G$. When to recommend compulsory versus optional CME programs? A study to establish criteria. Academic Medicine 1997;72 (9):760-764.

7. National Clinical Assessment Service. The Back on Track Framework for Further Training: Restoring Practitioners to Safe and Valued Practice. 2010. London, UK: National Clinical Assessment Service; https://kingsfund.blogs.com/health_management/2010/12/the-back-ontrack-framework-for-further-training-restoring-practitioners-to-safe-and-valued-practice.html. AccessedNovember 15, 2015.

8. Williams BW. The prevalence and special educational requirements of dyscompetent physicians. The Journal of Continuing Education in the Health Professions 2006;26 (3):173-191.

9. Alam A, Klemensberg J, Griesman J, Bell CM. The characteristics of physicians disciplined by professional colleges in Canada. Open Medicine 2011;5 (4):e166-e172.

10. Kohatsu ND, Gould D, Ross LK, Fox PJ. Characteristics associated with physician discipline: A case-control study. Archives of Internal Medicine 2004;164 (6):653-658.

11. Bismark MM, Spittal MJ, Gurrin LC, Ward M, Studdert DM. Identification of doctors at risk of recurrent complaints: A national study of healthcare complaints in Australia. BMJ Quality and Safety 2013;22 (7):532-540.

12. Shojania KG, Dixon-Woods M. 'Bad apples': Time to redefine as a type of systems problem? BMJ Quality and Safety 2013;22 (7):528-531.

13. van Mook WN, Gorter SL, De Grave WS, et al. Bad apples spoil the barrel: Addressing unprofessional behaviour. Medical Teacher 2010;32 (11):891-898.

14. Welch HG, Black WC, Fisher ES. Case-mix adjustment: Making bad apples look good. JAMA: Journal of the American Medical Association 1995;273 (10):772-773.

15. Grace ES, Wenghofer EF, Korinek EJ. Predictors of physician performance on competence assessment: Findings from CPEP, the Center for Personalized Education for Physicians. Academic Medicine 2014;89 (6):912-919.

16. Gabbay J, le May A. le May A. Practice made perfect: Discovering the roles of a community of general practice. In: Communities of Practice in Health and Social Care 2009: Hoboken, NJ: WileyBlackwell; 49-65. 
17. Polit DF, Beck CT. Generalization in quantitative and qualitative research: Myths and strategies. International Journal of Nursing Studies 2010;47 (11):1451-1458.

18. Malterud K, Siersma VD, Guassora AD. Sample size in qualitative interview studies: Guided by information power. Qualitative Health Research 2016;26 (13):1753-1760.

19. Varpio L, Ajjawi R, Monrouxe LV, O'Brien BC, Rees CE. Shedding the cobra effect: Problematising thematic emergence, triangulation, saturation and member checking. Medical Education 2017;51 (1):40-50.

20. Charmaz K. Constructing Grounded Theory 2nd edition. London, UK: Sage Publications 2014

21. Ross L. The intuitive psychologist and his shortcomings: Distortions in the attribution process. Advances in Experimental Social Psychology 1977;10:173-220.

22. Greenwald AG, Krieger LH. Implicit bias: Scientific foundations. California Law Review 2006;94: 945-967.

23. Coupland N. "Other" representation. Society and Language Use 2010;7:241-260.

24. LaDonna KA, Ginsburg S, Watling C. "Rising to the level of your incompetence": What physicians' self-assessment of their performance reveals about the imposter syndrome in medicine. Academic Medicine 2018;93 (5):763-768.

25. Lerner MJ, Miller DT. Just world research and the attribution process: Looking back and ahead. Psychological Bulletin 1978;85: (5)1030-1051.

26. Royal College of Physicians and Surgeons of Canada. CanMEDS: Better standards, better physicians, better care. http://www.royalcollege.ca/rcsite/canmeds/canmeds-framework-e Accessed July 12, 2019.

27. College of Family Physicians of Canada. CanMEDS-Family medicine 2017: A competency framework for family physicians across the continuum. https://www.cfpc.ca/canmedsfm/. Accessed July 12, 2019.

28. Molloy E, Bearman M. Embracing the tension between vulnerability and credibility:'Intellectual candour' in health professions education. Medical Education 2019;53 (1):32-41. 



\section{Educator, Judge, Public Defender: Conflicting roles for remediators of practicing physicians}

Gisèle Bourgeois-Law | Glenn Regehr | Pim W Teunissen | Lara Varpio 


\section{ABSTRACT}

Context Practicing physicians who remediate their peers face unique challenges. Recent research suggests that leaders of regulatory and educational institutions (i.e. those who might be seen as responsible for overseeing remediation programs for practicing physicians) view remediation as a duality: education and regulation. Research has yet to study the perspectives of remediators; therefore, to address that gap we asked: What is the nature of remediation as experienced by remediators?

Methods We used a theory-informing inductive data analysis study design with positioning theory as a sensitizing concept. We interviewed nine remediators from five Canadian provinces, asking them to narrate particularly memorable remediation experiences, then exploring the stories in more depth by asking probing questions around topics related to the research question. We used a hermeneutic analytic approach to explore the meanings that participants articulated about their remediation work by iteratively reading their stories, examining the sense-making that participants achieved through these narratives, and identifying the positions and responsibilities they described.

Results In their remediation narratives, participants variably positioned themselves in three different ways: educator, judge and public defender. For each position, remediators in turn framed the remediatee in a particular way. Participants shifted between educator, judge and public defender in response to evolving experiences with the remediatee, but they expressed preference for the educator position. However, they sometimes encountered serious obstacles to enacting that educator position. Those obstacles were imposed both by regulators and by remediatees.

Conclusion This study suggests that the duality of remediation as both education and regulation may be contributing to the challenges faced by those working one-onone with remediatees. Understanding the dual nature of remediation and equipping remediators with the tools to manage this duality might contribute to improving the experience for both remediators and remediatees, and ultimately to a greater number of successful remediation outcomes. 


\section{Introduction}

With intensified public demands for professional accountability and the ensuing development of revalidation programs, the need to provide remediation for practicing physicians is acute. However, researchers have only recently begun to grapple with the practical and conceptual issues related to the remediation of practicing physicians. Recent research has suggested that leaders of educational and regulatory institutions (i.e. those who might be seen as responsible for overseeing remediation programs for practicing physicians) hold some ambiguity with regard to their conceptualization of remediation. That is, they view remediation simultaneously as both an educational process and as a regulatory action (in that it involves usurping the remediatee's right to autonomously selfregulate decisions about their own learning activities). ${ }^{1}$ The tendency of individuals to shift between these two conceptualizations without explicit awareness might well be the source of several ongoing challenges in remediation, including the issues of what falls under the definition and who should be responsible for offering it. This raises questions regarding how this ambiguity might be experienced by remediators themselves, i.e. those who are tasked by regulatory and health authorities to oversee the learning and practice of physicians with significant competence gaps.

Despite extensive searches of the peer-reviewed literature, we were unable to locate any studies focused on how remediators of practicing physicians conceptualize themselves and their role. Given the paucity of literature at the practice level, we looked at how remediators at the residency level conceptualize what they do. However, the only study that engaged with remediators of residents explored how remediation should be conducted, not how they conceptualize their role. ${ }^{2}$ Kalet and Zabar imply a particular role for residency remediators by suggesting specific competencies for clinical educators conducting remediation, but this was based on expert opinion on different facets of remediation, and not on discussions with remediators. ${ }^{3}$ Notably, these papers focus on the development of skills such as feedback skills, assessment skills, and the skills required to develop remediation plans. Given this, it appears that remediators of residents are being positioned as an educator (specialized in the education of struggling learners). Consistent with this positioning, elsewhere, coaching and mentoring skills have been emphasized by those developing resident remediation programs, although it is not clear that actual remediators at the residency level have identified and aligned themselves with these educator, coach or mentor roles. ${ }^{4,5}$

Exploring how remediators, and in particular remediators of practicing physicians, conceptualize their role is important for ensuring that faculty development and professional support are appropriately targeted. Furthermore, insights into this role would enable us to better understand the nature of remediation at the practice level and the particular challenges faced in developing remediation programs for practicing physicians. As part of a larger program of research exploring social constructions of remediation in medicine, therefore, this study explored these remediators' understanding of the work they engage in and the role(s) they adopt therein. More specifically, our research question asked: What is the nature of remediation as experienced by remediators? 


\section{Theoretical Lens: Positioning Theory}

To explore these questions, we used a theory-informing inductive study design and analysis relying on positioning theory as a sensitizing concept..$^{6-9}$ In its social constructionist approach, positioning theory draws distinctions between roles and positions. It suggests that the roles individuals occupy don't change, but positions - how the individual enacts their roles - vary and are subject to conscious negotiation between the individual and their environment. Positioning theory highlights the "dynamic aspects of encounters in contrast to the way in which the use of 'role' serves to highlight static, formal and ritualistic aspects".10 Positioning theory addresses the implicit rights and duties of each individual in a given role to take certain positions thus both enabling and limiting the individual's ways of acting and speaking. For example, a physician remediating a peer might ascribe to him/ herself the right to speak to the remediatee in a way that other peers would not. However, the language used might vary at different times during the course of the remediation depending, at any given instant, on how the remediator wanted the remediatee to view them at that moment (eg as a support for the remediatee vs. an agent of the regulator). Positioning theory also suggests that Individuals not only position themselves but also others. For example, the remediator might variably position the remediatee as a learner or as a problematic physician, and ascribe to the remediatee the duty to respond to advice differently than they might expect a practice partner to do.

Another important concept in position theory is storyline. The rights and duties ascribed to individuals depend on the storyline of how an event or process is expected to unfold. Positions and storylines influence actions so that an individual's behavior is determined not by a response to a stimulus but rather by the interaction between what one is capable of doing and what the context, including other individuals and the stories told in that context, allow one to do. ${ }^{11}$ Thus, positioning theory enables us to understand how storylines and the way individuals use language to represent themselves to others can influence behaviors.

Since positioning theory emphasizes meaning, and the management of that meaning, it aligns well with our aim of understanding the nature of remediation - both with respect to the processes followed to realize remediation and the meanings individuals construct of remediation itself. ${ }^{10}$ By distinguishing between roles and positions, positioning theory enables us to perceive the difference between the abstract role of remediator and the moment-by-moment embodiment of that role.

\section{Methods}

We constructed this investigation using a narrative approach for several reasons. ${ }^{12}$ First, it provides a structured means by which to explore different aspects of remediation without imposing a structure on the phenomenon itself. ${ }^{13}$ Since there is a paucity of literature addressing the remediation of practicing physicians, we needed to engage in truly descriptive and exploratory research; a narrative approach supports such discoveryoriented inquiry. Second, narrative research goes beyond individual experience to consider how experience fits within relationships and the broader social context. ${ }^{14}$ These 
considerations are important to our research because remediation is situated within a particular medical culture and within a variety of jurisdictional contexts. Finally, our choice of positioning theory as a sensitizing concept suggested the use of narrative analysis. Positioning theory helps us understand how people describe themselves and what they do through the stories they tell. This aligns with the affordances of narrative analysis since it "reveals the normative constraints of a story-line", i.e. how individuals' actions and positions are limited by the stories they construct. ${ }^{10}$

To transparently report the foundational underpinning of our narrative approach, we explicitly reflected on each of Thomas's four questions for clarifying narrative investigations. ${ }^{15}$ These questions reference the study's underlying conceptual frameworks, epistemology, ethical stance, and methods. In our study, conceptually, we looked at the narratives through the sensitizing lenses of positioning theory to understand how our participants attempted to enact the roles of remediator of practicing physicians. Our epistemological stance was that of relative subjectivism i.e. that knowledge is the result of the interaction between researcher and participants, rather than derived from a hypothesis to be tested. ${ }^{16}$ Our ethical stance was that of an empathic colleague listening: the principal author and interviewer was for 10 years the director of a clinician assessment and remediation program and could relate to the stories she was hearing. The co-authors worked in close collaboration to ensure that the insights of participants were carefully considered in detail and that all analyses were collaboratively constructed. Methodologically, we collected data via one-on-one semi-structured interviews designed to evoke participant narratives about their experiences as a remediator and used a hermeneutic approach for analysis. ${ }^{17}$

\section{Participants and Interviews}

Since we sought perspectives from a wide range of remediators who worked in a diversity of contexts, but who were also connected through similar professional expectations, we decided to interview participants across Canada. In Canada, programs for the remediation of practicing physicians vary across provincial jurisdictions, but all physicians are expected to maintain competency standards set by national level organizations. We sampled participants from across provincial jurisdictions to obtain as wide a variety of experiences as possible. Contact information for Canadian remediators of practicing physicians is closely guarded; therefore, we worked with regulatory authorities and university offices involved in the remediation of practicing physicians to extend an invitation on our behalf to their remediators. All remediators who responded to our invitation were interviewed by phone, the discussions were audio recorded, and the audio files were transcribed by a professional transcriptionist and rendered anonymous.

In the interview, participants were asked to narrate particularly memorable remediation experiences. When these stories were positive (i.e. successful in the eyes of the remediators), follow up questions asked participants to share a second story that was less successful. The inverse was asked when negative stories were offered initially. The interviewer helped participants to explore the stories in more depth by asking probing questions around topics related to the research question. 


\section{Analysis}

We used a hermeneutic approach to analyse the narrative, exploring the meanings that participants articulated about their remediation work by iteratively reading their stories, examining the sense-making that participants achieved through these narratives, and identifying the positions and responsibilities they described. ${ }^{17}$ This was aligned with our overall research design, which aimed to understand the content of the stories and the meanings participants' ascribed to elements of their narratives.

GBL conducted the interviews, read all the transcripts and independently constructed initial interpretations. LV read four of the transcripts, and independently developed preliminary interpretations. GBL and LV met to discuss and confirm data interpretations, developing a shared understanding of the data and analysis directions. GBL re-read all transcripts and coded the data following the direction collaboratively developed. The entire research team met on a regular basis throughout the analytic process to discuss the ongoing interpretation of the readings, and the composition of the analysis.

\section{Reflexivity}

GBL is a retired physician doing her $\mathrm{PhD}$ on conceptualizations of remediation for practicing physicians. Her past experience as the director of a clinician assessment and remediation program enriched her understanding of the data, but also required ongoing probing by her supervisors (the other 3 authors) to avoid preconceptions unduly influencing her understanding of the data. PWT is a gynecologist with a PhD in health professions education and extensive experience supervising $\mathrm{PhD}$ students doing both quantitative and qualitative work. LV is a research scientist with expertise in qualitative research who has investigated health professions education for over a decade. GR is a PhD trained researcher with extensive experience in the field of health professions education conducting and supporting both quantitative and qualitative scholarship.

The study received ethical approval from the UBC Behavioral Research Ethics Board (protocol \#H18030).

\section{Results}

Nine individuals, from five Canadian provinces, accepted that invitation to participate. Participant demographics reflected their widely varying experiences as remediators. The participants had remediated from one to over one hundred physicians and had been involved in remediation of practicing physicians from one to over 20 years. They held nine different titles in their remediation activities that seemed to have no direct link to remediation expectations. In some cases, participants held more than one formal title within the regulatory authority. For example, practice advisors and professional conduct assessors also engaged in the remediation of practicing physicians. Only one participant had received specific training for their remediation activities.

Participants' interactions with their physician remediatees varied widely, from a onetime phone call intervention to ongoing face-to-face contacts that lasted over several 
months. Five participants had spent time in a remediatee's practice. Two participants described working with a remediatee using standardized patients to further define and remediate the problem. Two participants had only engaged in phone conversations with remediatees. Remediatee problems included poor charting, poor communication skills that led to adverse events, poor technical skills with increased complications, and generalized clinical competence deficits.

While narrating their unfolding remediation stories, participants seemed to variably position themselves in three different ways: educator, judge and public defender. For each position, remediators in turn framed the remediatee in a particular way.

\section{Positions}

\section{Remediator as Educator}

When positioning themselves as educators, participants described their activities as guiding the remediatee to make required practice changes. Remediators worked to develop an educational alliance with the remediatee, to involve the remediatee in decisions, and to give/preserve as much remediatee autonomy as possible. ${ }^{18}$

While they considered being quite directive as part of that work - e.g. "we got him to... [do $\mathrm{x}$ ]" (P1) - remediators also considered the impact of the required change, and attempted to facilitate that change in many ways including identifying resources, facilitating connections, and being supportive. They worked to empower remediatees to make needed changes:

I saw my role ... just to, you know, kind of let him know about some of the resources that he wasn't aware of ... some of the changes that had occurred while he wasn't practicing. (P4)

So the remediation was more around building his confidence, helping him with time management, helping him set limits with patients. (P6)

When positioning themselves as educators, our participants framed the remediatee as a colleague in need of help because of circumstances, as individuals who were not that different from themselves.

I guess when I first thought about physicians that need remediation, ...you know, you think probably they're not good physicians or they're doing something wrong. But going through the process, I don't think that was the case at all. I think it's an easy thing to kind of run into these bad habits especially if you are busy. And then you don't really have the same external forces like when you're at residency or if you're not in a group practice and other people are doing the same thing you're doing, it's easier to kind of start adopting some of these habits that are not the best. (P5)

\section{Remediator as Judge}

When positioning themselves as judge, participants described their role as adjudicating whether the remediatee was ready to return to unsupported practice. Unlike assessing, 
an activity all educators do at some level to determine the kinds of support an individual needs, the position of judge required the remediator to make a summative judgment on an individual's competence, thereby ascertaining if remediation has (yet) achieved success and therefore is complete. In other words, the remediator as judge needed to rule if the remediatee was ready to practice autonomously. This responsibility weighed heavily on participants, especially when there was limited interaction with the remediatee:

So that felt a lot more responsibility because if you say that he's okay then he'll be seeing patients. (P5)

there's no measurable outcome particularly other than my impression of whether they're going to change their practice. (P8)

When positioning themselves as judges, remediators tended to frame remediatees as akin to final year residents (i.e. this was the competency standard to be achieved, or at least to approximate). Consequently, there was a degree of inequality in the relationship; the remediatee was not at the level of a peer:

(He would) sort of try to have conversations with me at a consultant level that I have with my other consultants-- colleagues. ... But because his knowledge and skill level was so deficient, him trying to have consultant level conversations with me just didn't work. (P9)

\section{Remediator as Public Defender}

When remediation did not progress successfully, remediators positioned themselves as defenders of the social contract between the medical profession and the public. When positioning themselves as public defenders, remediators described their position as ensuring that the public was protected from dangerous and/or incompetent practitioners and as protecting the profession's ability to self-regulate.

I want to make sure doctors are good doctors. And I want us to have a good reputation in the community and I would like us to continue to self-regulate. And in many ways if there are a lot of doctors out there like this particular physician then I think we're in big trouble and we are at risk of losing our ability to self-regulate. (P9)

The public defender position was invoked when the remediator began to question whether the remediate could ever reach the standards of professional performance. Thus, when remediators took on this position, they tended to position remediatees as recalcitrant, unrepentant, and unprofessional individuals who deserved their fates. To be clear, remediators did not position all remediatees this way; rather, this was how remediatees were positioned when efforts at educating them were failing or were completely thwarted.

three weeks of very difficult clinical experiences in which he tried to pretend he had knowledge he didn't have (P9) 
Then, the relationship with the remediatee could be highly charged by a lack of trust on the remediatee's part, and frustration for the remediator:

the physician showed up with his lawyer and demanded that his lawyer not allow me to criticize him or criticize his practice or anything like that. (P1)

\section{Positioning obstacles and struggles}

Participants expressed preference for the educator position: they engaged in remediation processes to help remediatees improve their practice. However, they sometimes encountered serious obstacles to enacting their educator position. Those obstacles were imposed variously by regulators and by remediatees.

\section{Limitations imposed by the regulator}

Participants described regulatory authorities as generally positioning them as educator or some variation thereof (e.g. coach, mentor, preceptor). However, remediators felt they were not always given the tools required to fully enact that position. For example, unless remediators were involved in the original assessment or decision that led to the remediation, they frequently could not access the information necessary to address the problem(s) that instigated the process:

I'm not privy to the complaint, really. Only just sort of a sketch.... So part of the issue for me was that the (first meeting with the physician) happened right before we started the session... The cases selected for the first session did not address the doctor's stated goals. (P8)

Remediators also had to contend with limitations imposed by the regulatory authority over the conduct of the remediation. For example, regulatory processes constrained what they could do in terms of time and scope:

Participant: "I would have liked to continue on longer to see how the person progressed." Researcher: "So who decided how many sessions and when things ended?" Participant: "The College did."(P4)

As an additional complication, although the explicit message from the regulatory authorities positioned the remediators as educators, the remediators suggested that they heard an implicit message to (also) position themselves as judge and gatekeeper to varying degrees and with varying authority, leading to ambiguity regarding how to balance these various positions:

I would want to tease out the assessment and preceptorship piece a lot more specifically. And probably try and separate those more than I was supposed to for this particular case (P9)

This limited their ability to effectively adopt any position. While they strove to be supportive, even when expected to be judge, they remained aware of the dangers inherent in trying to balance different positions: 
that was absolutely part of the challenge in that if it had just been strict assessment, I think that would have been a lot easier in having a defined role for a defined timeframe...(P9)

The lack of information regarding regulatory processes and/or the circumstances regarding the individual remediation case may have led to their questioning the ultimate purpose of the remediation. Participants raised concerns that the process was a "rubberstamp" (P8) and that the regulating bodies would ignore the preceptor's report (P9).

\section{Limitations imposed by the remediatee}

Positions imply storylines. That is, the position one assumes or accepts is usually accompanied by a narrative that one expects or wishes to unfold. The ideal storyline in remediation is that the remediatee ultimately comes to accept the situation and, having recognized the problem(s), turns their practice around and becomes the physician they were meant to be:

"Seeing other docs improve their performance and being proud of that and then being grateful and saying things like: 'Boy, when I first met you I was scared and I thought this was going to be a horrible thing. But, man, it was really worthwhile and I'm really glad we got together and that you took the time to work with me on this. 'Cause it's certainly helped me.'I get somebody who says that, I feel pretty good. "(P1)

However, positioning is not a one-way street. ${ }^{19}$ The remediatee could implicitly or explicitly refuse their assigned position. When this happened, the remediator was forced into a more adversarial position ("I'm the five-foot-tall female bad guy" (P8)), and often found themselves switching to a more authoritarian position to exert authority ("There's a reason why I'm in this position and you're in that position"(P4)). By persisting in positioning the remediator as an adversary instead of as an educator, the remediatee could force the remediator to switch their focus from empowering and supporting a struggling colleague to protecting the vulnerable public. Remediators, therefore, struggled to maintain their position as educators in the face of remediatees who refused to accept them as such.

Age and gender could also have an impact on how remediatees responded to remediators. Female participants described struggling to establish authority and credibility with older male remediatees:

So there was a power differential in that I [female remediator] was the preceptor. But there's also a power differential in that he had been practicing longer than me. And I think felt also he had his feelings around having to go through this process and having me, who was younger and less experienced, be his preceptor, I think, was a big challenge. And there was probably a bit of a gender power differential there as well. (P9)

Further, the way remediators positioned remediatees was not fixed. Remediatees could start out angry and defensive but could change to see that the remediator was there to help them (we did not hear stories of the inverse, i.e. where a remediatee went from accepting to uncooperative): 
so it took a little bit of time to kind of get her to see me as a person that's trying to help her instead of someone who might be, you know, causing her to lose her livelihood or causing her to lose her license. (P5)

Interestingly, our participants noted that their previous experiences as an educator and remediator of trainees did not fully prepare them for the challenges in negotiating being an educator for colleagues.

I'm used to being the one with the power and that being generally acknowledged by learners. But when it comes to preceptoring your colleagues that's trickier. And you kind of have to figure out ways to navigate that. (P9)

\section{Discussion}

The aim of this study was to deepen our understanding of remediation by listening to the stories of those who directly remediate physicians. The results of a previous study had suggested to us that remediation in practicing physicians can be viewed as both an educational process and a regulatory activity, and that failure to appreciate the inherent contradictions in this duality might be contributing to the profession's difficulties in addressing poor performance. ${ }^{1}$ The results of the present study suggest that failure to articulate, appreciate, and deal with this duality might also be contributing to challenges faced by those who work directly with struggling physicians. Positioning theory enabled us to see that our remediators strove to manage the meaning of the remediation process for themselves, and for those whom they were asked to remediate, by focusing on the educational component and ignoring as best they could the implied loss of professional autonomy. That positioning was difficult to sustain if remediatees were unwilling to accept either the remediator's position or the implications of the remediatee position.

An underlying question at the beginning of this program of research on the nature of remediation in practicing physicians was whether remediation at the practice level is qualitatively different than remediation at the undergraduate or postgraduate education levels. Thus, we might well ask whether this effort at positioning is unique to remediation in the CPD world. We would posit that one important difference is that only at the practice level do remediators have to contend with the aforementioned duality; only at the practice level do remediatees lose their professional autonomy. Remediatees at the undergraduate and postgraduate levels do not have, nor do they expect to have, full educational and clinical autonomy. Remediators at the practice level thus face a unique challenge - to make this hopefully temporary loss of professional autonomy palatable to the remediatee so that the necessary learning (defined as per the behaviorists as a permanent change in behavior) can take place. Thus, we might speculate that they face greater challenges in developing the necessary educational alliance. ${ }^{18}$

Although there is no study on how residency educators position themselves, Chou et al do discuss the need for balancing empathy for the struggling individual with the profession's responsibility to future patients and to society, which could be construed as a 
variation of balancing educator and public defender positions. ${ }^{20}$ However, it is only at the undergraduate level that we find a study that explicitly uses positioning theory to look at how educators position themselves in relation to struggling learners. Hu et al in their study on positioning in medical student support encounters, note that educators struggle with contradictory aims of support and sanction; our respondents similarly struggled between educator and public defender roles. ${ }^{21}$ The undergraduate educators, not surprisingly, used metaphors from parenting that were absent in our peer remediators. As did our respondents, Hu's participants generally shifted between positions, often unconsciously. Unlike our participants, however, some of Hu's respondents were fixed in one or other of either judge or confidant "as if it were a personal philosophy for student support". Whether this is unique to trainee remediation or a characteristic of remediators that would also apply at the practice level but that our study did not uncover is unclear. It is, however, unlikely that practice remediators who adopted a fixed position e.g. educator, would be asked by the regulatory authorities to remediate on a recurring basis.

Remediators at the undergraduate level sometimes struggled with resolving tensions between positions, in their case between assessment and support. They were either unable to resolve those tensions or resolved them by accepting that they couldn't effect change unless the student desired it. Our peer remediators were in a sense more fortunate because they had neither been responsible for calling for remediation, nor were they ultimately responsible for the final decision, even when the regulator depended on their report or recommendation. They were thus able to resolve the tension between educator and public defender by referring the remediatee back to the regulatory authority. It was no longer their problem, which is not to say that it didn't leave them sometimes wishing they had been more successful and wondering what they might have done differently.

\section{Implications}

Our results suggest that our remediator participants experienced remediation as both education and regulation, although they may not have expressed it in those terms. Remediation at the practice level requires remediators to move skillfully between several positions that include varying degrees of educational and regulatory components. Our participants were more comfortable with the educator position, particularly those remediators who were fairly new at the process, however, they felt somewhat unprepared even for this role, as prior experience remediating trainees did not adequately equip them to work with practicing physicians. A framework that enables them to understand the dual nature of remediation at the practice level might prove helpful. Faculty development regarding the different positions they may need to adopt in their role of remediator may also be helpful. Finally, preparation about when and how to transition between positions might enable remediators to more skillfully manage the dual aspects of education and regulation.

\section{Limitations}

We were unable to recruit in Québec - one of Canada's two largest provinces, and the one with the most organized remediation program - because of regulatory authority rules that forbade their preceptors from speaking with us. However, we did recruit participants from across Canada in five provinces, so our data includes a breadth of programs and 
experience. Narrative research of necessity is limited to small numbers of participants who choose to tell their stories; it allows us to uncover the meaning of the experience. Since the overall aim in this research was to uncover the meaning of remediation, we suggest that our small population pool is an acceptable limitation. To better understand the actual process of remediation, it might be helpful to use the insights from this study to conduct focus groups using semi-structured questionnaires.

\section{Conclusion}

Remediation in practicing physicians includes both educational and regulatory aspects. This study suggests that this generally undiscussed duality may be contributing to the challenges faced by those working one-on-one with remediatees. Understanding the dual nature of remediation and equipping remediators with the tools to manage this duality might contribute to improving the experience for both remediators and remediatees, and ultimately to a greater number of successful remediation outcomes. 


\section{References}

1. Bourgeois-Law G, Varpio L, Regehr G, Teunissen PW. Education or regulation? Exploring our underlying conceptualisations of remediation for practising physicians. Medical Education 2019;53 (3):276-284.

2. Krzyzaniak SM. A qualitative study of medical educators' perspectives on remediation: Adopting a holistic approach to struggling residents. Medical Teacher 2017;39 (9):967-974.

3. Kalet A,and Zabar S. Preparing to Conduct Remediaion in Kalet A, Chou C. Remediation in Medical Education: A Mid-Course Correction. New York: Springer; 2014.

4. Kalet A, Chou C. Remediation in Medical Education: A Mid-Course Correction. New York: Springer; 2014. p24

5. Shearer C, Bosma M, Bergin F, Sargeant J, Warren A. Remediation in Canadian medical residency programs: Established and emerging best practices. Medical Teacher 2019:41 (1):28-35.

6. Young M, Varpio L, Uitjtdehaage S, Paradis E. The Spectrum of Inductive and Deductive Research Approaches Using Quantitative and Qualitative Data. Academic Medicine 2019, December 10 Published ahead of print.

7. Davies B, Harré R. Positioning: The Discursive Production of Selves. Journal for the Theory of Social Behaviour 1990;20 (1):43-63.

8. Harré R, Moghaddam FM. The self and others: Positioning individuals and groups in personal, political, and cultural contexts: Greenwood Publishing Group; 2003.

9. Lagenhove L, Harré R. Positioning theory: Moral contexts of intentional action. Oxford, UK: Blackwell; 1999.

10. Harré R, Moghaddam FM, Cairnie TP, Rothbart D, Sabat SR. Recent advances in positioning theory. Theory \& psychology 2009;19 (1):5-31.

11. Harré $R$, and Moghaddam M. The self and others in traditional psychology and in positioning theory. In The self and others: Positioning individuals and groups in personal, political, and cultural contexts: Greenwood Publishing Group; 2003.

12. Chase S. Narrative inquiry:Toward theoretical and methodological maturity. The SAGE handbook of qualitative research. 2018:546-60.

13. Charmaz K, McMullen LM. Five ways of doing qualitative analysis: Phenomenological psychology, grounded theory, discourse analysis, narrative research, and intuitive inquiry: Guilford Press; 2011.

14. Clandinin DJ, Cave MT, Berendonk C. Narrative inquiry: a relational research methodology for medical education. Medical Education. 2017;51 (1):89-96.

15. Thomas $C$. Negotiating the contested terrain of narrative methods in illness contexts. Sociology of health \& illness. 2010;32 (4):647-60.

16. Bergman E, de Feijter J, Frambach J, Godefrooij M. AM Last Page. Academic Medicine.2017;87 (4):545.

17. Josselson R. The hermeneutics of faith and the hermeneutics of suspicion. Narrative Inquiry 2004;14 (1):1-28.

18. Telio S, Regehr G, Ajjawi R. Feedback and the educational alliance: examining credibility judgments and their consequences. Medical Education 2016;50 (9):933-42.

19. Depperman, A. Positioning. In De Fina A, Georgakopoulou A. The handbook of narrative analysis: John Wiley \& Sons; 2019.

20. Chou CL, Kalet A, Costa MJ, Cleland J, Winston K. Guidelines: The dos, don'ts and don't knows of remediation in medical education. Perspectives on Medical Education 2019;8 (6):322-338. 
21. Hu WC, Woodward-Kron R, Flynn E. Educator as Diagnostician, Judge and Confidant: a positioning analysis of medical student support encounters. Advances in Health Sciences Education 2019;24 (4):707-724. 



\section{What's in a Name?}

Gisèle Bourgeois-Law

Invited Commentary; Perspectives on Medical Education 2019; 8 (6):320-321 

The authors of 'Guidelines: The do's, don'ts and don't knows of remediation in medical education' have provided us not only with guidelines for those wishing to develop or improve a remediation program in their institution, but also with a summary of what is currently known on the remediation of medical learners. ${ }^{1}$ This paper builds on previous work by several of the authors regarding emerging best practices for remediation programs. $^{2,3}$ In addition to incorporating new knowledge on remediation in the 5 years since Kalet and Chou's seminal book, the present guidelines have been refined to distinguish between systems level and individual level processes. We might perhaps think of this as a variation of 'think globally, act locally' - 'think systemically, act individually'.

The first challenge in grappling with remediation, as with any wicked problem, is how to define it. The authors define remediation as 'the act of facilitating a correction for trainees who started out on the journey towards becoming a physician but have moved off course.' Since no medical trajectory is perfectly straight, we might ask how far off course a learner must deviate before correction is mandated. There are also varying degrees and means of correction; how extensive or formal must these be to be classified as remediation? As an example, a recent study notes that some preceptors consider providing constructive feedback to be 'remediation'; since every learner benefits from constructive feedback at some point, this suggests that every learner will undergo some degree of remediation. ${ }^{4}$ Perhaps then, remediation might be further conceptualized as a continuum of support ranging from feedback to a formal structured experience.

However, the trigger for formal remediation depends not only on the trainee's precise location on the continuum of needed support but also, as Krzyzaniak et al. have noted, on the individual's response to corrective feedback. ${ }^{4}$ Two individuals with the same knowledge or skills gaps might respond quite differently to constructive feedback or offers of support, with one taking feedback to heart and diligently striving to improve, while the other persists in excuses and denial. The decision to mandate remediation would thus depend as much on the individual's response as on the size of the learning or skills gap. Where then, among the infinite possible combinations of course deviation and response to feedback, could one draw a line beyond which corrective actions constitute remediation?

Remediation, whether conceptualized as a correction for a few or a continuum of support for all, is highly fraught. Chou et al. suggest in the first guideline that remediation be reframed as 'a special zone of learning, self-improvement, personal development, resilience building, and an opportunity to practice with feedback.' Were remediation advertised as such, one might even expect eager learners volunteering to participate. But will reframing remediation remove the stigma, or is this yet another area where the hidden curriculum lurks? What is so negative about needing remediation that learners not only fear it but also distance themselves from peers who require it? As a postgraduate dean told us in a recent study: 'I think we're brutal. Frankly, I see it even in medical students and residents. If you start showing any signs of struggle or weakness the first instinct of many physicians is just to want you to be gone'.5 'Othering' our struggling colleagues thus starts early. In such a culture, no matter what we call it or how we frame it, remediation will continue to be problematic. 
Medicine has a strong culture of performance, a culture that leads trainees to view feedback meant to be formative as summative and thus threatening. ${ }^{6}$ If feedback is threatening, remediation must be catastrophic. In a profession where the goal is not only performance but independent performance - to be 'entrusted' to perform independently - independence in one's learning is the first expected step towards full professional status. Is it possible that by explicitly encouraging our students and residents to be selfregulated, independent learners we might inadvertently be increasing remediation's stigma? Remediation, by definition, is a failure of self-regulated learning: the learner must be told what, when and how to learn, and will be observed and/or tested to ensure that the learning has taken place. Reframing remediation as a'special zone of learning', or even as 'support' can't change that underlying connotation, nor will increasing the numbers of learners who are required to undergo the process. It might even, as we have seen in the world of continuing professional development, lead some learners to conflate general support or improvement strategies with remediation and refuse to engage with them.

Perhaps then, in conjunction with moving towards a culture of improvement rather than performance, we might also need to move from a learning culture of self-regulation to one of co-regulation. ${ }^{6}$ Having been involved both directly and indirectly in the remediation of practising physicians for many years, this commenter would like to suggest that the most dangerous physicians, and the ones most likely to lose their license to practice, are not those who fail to self-regulate their learning or to perform independently. They are, rather, those who refuse to listen to and incorporate feedback, whether from their patients, their colleagues, or their environment. They are those who fail to seek out or accept co-regulation and who persist in viewing themselves as fully autonomous, selfonly regulated professionals.

The questions in the paper's final paragraph highlight that the discussion around remediation is ultimately a discussion around values. It is also ultimately a discussion around culture, since our culture determines our values. It may be time to move beyond a culture of individual self-regulation to one of community co-regulation. Needing input and support from one's peers and teachers in training, and from the broader community once in practice, should not be a source of shame or lead to stigmatization. The only stigma should lie in the refusal to accept feedback and needed support in order to improve. If we can get to that place, what we call or how we define remediation may ultimately be a moot point. 


\section{References}

1. Chou CL, Kalet A, Costa MJ, Cleland J, Winston K. Guidelines: the do's, don'ts and don't knows of remediation in medical education. Perspectives on Medical Education 2019;8 (6):322-228.

2. Kalet A, Chou C. Remediation in medical education: a mid-course correction. Heidelberg, Berlin, New York: Springer; 2014. p. 24.

3. Cleland J, Leggett H, Sanders J, Costa MJ, Patel R, Moffat M. The remediation challenge: theoretical and methodological insights from a systematic review. Medical Education 2013;47 (3):242-251.

4. Krzyzaniak SM. A qualitative study of medical educators' perspectives on remediation: adopting a holistic approach to struggling residents. Medical Teacher 2017;39 (9):967-964.

5. Bourgeois-Law G, Teunissen PW, Varpio L, Regehr G. Attitudes towards physicians requiring remediation: one-of-us or not-like-us? Academic Medicine 2019;94 (11S):S36-S41.

6. Watling CJ, Ginsburg S. Assessment, feedback and the alchemy of learning. Medical Education 2019;53 (1):76-85. 

Discussion 
How we think about a problem impacts how we go about looking for ways to manage it, to whom we assign responsibility for working on solutions, who we seek to involve, and whether we think it is any of our concern. Thus, understanding conceptualizations of remediation is crucial for helping the profession advance in managing it. It also has broader implications. If we believe that remediation is or should be a component of the continuing medical education (CME) system, how we conceptualize remediation affects how we organize CME; the CME system in turn affects how we conceptualize remediation. In this thesis, I focused on the remediation of clinical competence issues because that appears to be the aspect the profession is struggling with the most, even though theoretically these concerns should be easier to remediate than professionalism lapses or health issues. Understanding why the profession struggles with remediation for clinical competence was a central impetus for this thesis.

\section{Summary of findings}

My synthesis of the literature (chapter 2), conducted at the beginning of the PhD journey, suggested that remediation is conceptualized as an educational process, with the final aim of returning the individual to self-regulated, autonomous practice. Consistent with that educational perspective, remediation appeared to be conceptualized as similar to remediation in residency with similar methods employed and similar remediator attributes and skills required. In regards to the individual requiring remediation (i.e. the remediatee), the literature focuses on understanding personal risk factors for poor practice. At least until now, this focus has been more in the service of identifying these individuals so that their practice can be assessed than in service of determining how to support at risk individuals before problems develop. Finally, the literature on medical culture suggested that aspects of that culture, including shaming and blaming individuals, the reluctance to challenge a colleague's practice, and the concept of professional autonomy might all affect to a greater or lesser degree the willingness to remediate. ${ }^{1,2}$

My empirical studies then explored how the conceptualizations and attitudes evidenced in the literature manifest in those directly and indirectly involved in remediation. The first study (chapter 3) showed that remediation stakeholders conceptualize remediation as having both educational and regulatory aspects and that they slip unconsciously between these two conceptualizations. This may help explain why universities hesitate to accept remediation as part of their mandate: they are focusing on remediation's regulatory aspect. On the other hand, regulatory authorities, those responsible for public protection, deem their main role to be assessment and consider remediation to be the purview of educators, i.e. of the university. Failure to come to grips with the education/regulation duality not only makes organizing remediation more difficult, it also leads to practice improvement initiatives being misconstrued as regulatory by the general physician workforce.

In addition to ambiguous conceptualizations of remediation, the first empirical study showed that stakeholders sometimes demonstrate ambivalence towards their struggling colleagues and about whether remediation, versus removal of licensure, is a wise use of scarce resources (chapter 4). Our participants suggested that this ambivalence starts early 
in training - students and residents tend to distance themselves from peers they perceive as struggling. This tendency to 'other' struggling colleagues can be explained by both various psychological theories (elaborated below) and by the medical culture of autonomy: the individual is solely responsible for developing and maintaining competence.

In my second empirical study, (chapter 5) I explored how peers tasked with working oneon-one with physicians identified as having clinical competence issues might navigate the competing educational and regulatory responsibilities implicit in the remediation mandate. An analysis of their narratives suggested that failure to acknowledge the education/regulation duality and to train remediators to identify and move between different positions in the remediator role may be contributing to making the remediation experience more challenging for remediators than it needs to be.

In sum, the empirical studies we conducted confirmed that remediation is being explicitly addressed primarily as an educational process, and they further refined our knowledge by exploring the regulatory component that is not always explicitly acknowledged. We saw that failure to acknowledge and manage this duality can contribute to challenges for peer remediators. Finally, whereas the literature focuses on the characteristics of physicians requiring remediation, the findings from this thesis research suggest that a focus on characteristics/traits could lead to 'othering'.

The aim of this thesis was to explore how the various stakeholder groups conceptualize the remediation of clinical competence in practicing physicians and how these various conceptualizations hinder or facilitate the process of addressing identified performance concerns related to knowledge and skills i.e. to explore previously unidentified barriers to involvement with remediation. The discussion will thus first look at what our research suggests regarding these barriers. I will then briefly discuss remediation in practice versus that in residency. I began the PhD with the firm conviction that they are different, but with only a vague notion as to why that might be. I ended by realizing that they are in many ways quite similar, but that the important differences may help explain some of the challenges of remediation in practice. Finally, I will discuss the dual nature of remediation in practice, the implications of that duality, and how we might manage it. Failure to manage that duality may be the biggest barrier of all to involvement in remediation.

\section{Barriers to the profession's full involvement in remediation}

Our research suggests that two significant barriers to involvement in remediation may be related to 1) how physicians requiring remediation are viewed by members of the community, and 2) how remediation is conceptualized.

The tendency to view those who require remediation as 'other', while perhaps not surprising, has not been previously discussed in the literature. It may also be a critical barrier to individual and organizational involvement with remediation. If physicians requiring remediation are not 'one-of-us' (i.e. not perceived as being a member of the medical profession), professional associations, universities, and regulators may be less 
likely to see themselves as responsible for remediation. Interestingly, othering appears to be more likely, although not exclusively, to occur in those physicians who have had limited contact with remediatees. For example, it was in our organizational stakeholders, who might not have had direct involvement with the remediation process, that we frequently saw implicit othering comingled with their descriptions of these physicians being victims of circumstance ('There but for the grace of God go l'). By contrast, our remediator participants didn't 'other' remediatees unless the physicians requiring remediation persisted in refusing to improve. Hearing remediatee stories offered remediators insight into how easily circumstances can lead a physician to find him/herself in a situation where remediation is invoked. While an argument can be made that medical culture is a root cause promoting the othering of colleagues in difficulty, psychological theories such as attribution theory and the just world phenomenon may also be at play, as we suggest in chapter 4.,4 The comment from a participant that "as soon as you show signs of struggle, the reaction of many is just to want you to be gone" appears at first glance to contradict the commonly held assumption that physicians 'stick together'. If struggling physicians across the continuum are perceived as 'not-like us' and that they can 'no longer be part of this group', as we saw in chapter 4, then might too close a relationship with such individuals be seen as a risk for also being viewed as 'not-like-us', for being damned by association? Doctors may like to think we are special, but in this case, I would suggest that we are behaving like everyone else.

Interestingly, othering is not necessarily negative. Canales defines othering as "the process of how we engage with those we perceive as different from ourselves" and asserts that it can be both exclusionary and inclusionary. Inclusionary othering uses power of the dominant person or group to transform the experience of others. Canales suggests that inclusionary othering starts with being able to view the world from the other's perspective. Comments such as "there but for the grace of God go I" or "anyone can run into trouble for any number of reasons" could be viewed as inclusionary othering. On the other hand, comments such as "Maybe you just don't have it in you to be what you need to be" or "normal people with a reasonable degree of insight might possibly respond to circumstances differently" could be viewed as exclusionary. Those latter comments suggest that there is no way the individuals described could ever be included in our particular group. Inclusionary versus exclusionary othering thus offers another way to frame the 'one-of-us' / 'not-like-us' dichotomy we saw in chapter 4.

I would suggest that this inclusionary othering often happens in a successful remediation experience. The remediator sees that the remediatee is not like them or like most physicians. They see the remediatee struggle with aspects of their practice that the remediator manages without difficulty, such as record-keeping, avoiding missing important lab test results, or keeping up to date with treatment modalities. The remediator then uses the power conferred on them by the regulatory authority as well as the power of their own knowledge and experience to coach and guide the remediatee to adopt more effective habits so that they can rejoin the fraternity of competent physicians. What is less clear is what happens after successful remediation. Is the successfully remediated physician viewed as no different from one who has never had to undergo remediation? Alternatively, does the remediation experience result in what Goffman terms "not the acquisition of fully 
normal status, but a transformation of self from someone with a particular blemish to some with a record of having corrected a particular blemish"? ${ }^{6}$ The answer to that question might depend on who one asks (i.e. stakeholder, remediator, remediatee, or general physician public). Our research did not suggest an answer, but this is a question to bear in mind for future studies.

Canales also suggests that one way othering can be inclusionary is to use a language to describe these others based on what these others themselves would prefer. Perhaps asking remediatees what their preferred term would be for the process they are required to undergo would help decrease their perceived stigma. Finally, as a nursing instructor, Canales was interested in how instructors might engage with students from 'othered' backgrounds. Her description of teaching as 'being with' rather than 'doing for' is reminiscent of our remediator participant who described remediation as "walking the road with" the remediatee. The concept of inclusionary othering thus bears consideration in the organization of remediation in practice.

I would now like to turn to the second barrier, and a more extensive discussion of conceptualizations of remediation as a potential barrier. One cannot talk about conceptualizations of remediation without first talking about conceptualizations of competence, as our conceptions of competence influence our conceptions of remediation. Brian Hodges has suggested five discourses of competence and noted how each of these different discourses leads to a different focus of assessment and associated different dangers. ' Of the five discourses identified by Hodges, all but the psychometric discourse have been applied to the remediation of practicing physicians, albeit not explicitly identified as such. For example, giving physicians guidelines to read and courses to attend would arise from the Knowledge discourse, where competence is defined as mastering a body of knowledge, and the physician requiring remediation is deemed to have a knowledge gap. Where remediation focuses on changing observable behaviors, the underlying discourse is that of Performance, in which competence is expressed through performance, and dyscompetence is thought to arise when relevant knowledge is not integrated with performance. When remediation includes a focus on improving self-regulated learning and self-assessment, it indicates that the Reflection discourse is at play; the individual is deemed to have failed in self-reflecting and identifying their own learning gaps. Finally, when competence is demonstrated by meeting standards of quality assurance and audits, the Production discourse, remediation may be based on the individual attaining institutional performance metrics, or meeting quality improvement goals.

These four competence discourses were at play to a varying degree in the remediation stories we heard from remediators (chapter 4). The Knowledge and Performance discourses may be more evident when the focus is on remediation as education, and the Reflection and Production discourses may be more evident when the focus is on remediation as a regulatory issue. The potential need to integrate education and regulation to address remediation suggests that we may also need to integrate these four discourses to adequately address remediation. That is to say, remediation should fill knowledge gaps, ensure competent performance, help prevent future problems by developing reflective 
and self-directive skills, and ensure that current standards of practice are followed. Otherwise, we may find ourselves encountering some of the dangers inherent in focusing on only one discourse. As an example, in more than one remediator story, the individual was under the distinct impression that the remediatee was playing a game, doing and saying what they thought the remediator wanted to hear and see. This led the remediator to question the remediatee's ability to realize long-term change. The remediatee was focused on demonstrating performance, when perhaps the development of reflection skills was just as crucial.

The above discourses of competence all relate to individual, rather than to collective, competence and do not take into account systemic influences. ${ }^{8}$ The tendency to view dyscompetence and remediation as individual rather than systemic problems, as noted in chapter 2 on our discussion of the social determinants of competence, may be contributing to an important barrier related to how remediation is conceptualized. If remediation is an individual's problem, no one institution views remediation of that individual as clearly linked to their institutional mandate. Not all regulatory authorities consider remediation part of their role. ${ }^{9}$ Neither do many universities, in spite of the efforts of some regulators to frame remediation as 'after-sales service' i.e. a responsibility of the program that trained the physician in the first place. We struggled to identify Deans of Medicine to interview; the only one who agreed, not surprisingly, came from the university where involvement in remediation has been ongoing for almost three decades. One Dean was quite adamant and almost rude (using capital letters in part of his email response) that this was the health authority's problem, not the university's.

These barriers are of course not the only barriers to institutional and individual involvement in remediation. A potentially helpful framework to identify these barriers may be found in Moser and Ekstrom's framework developed to identify barriers to climate change adaptation. ${ }^{10}$ Our research suggested a similar tendency as occurs in climate change - to deny the extent of the problem and to push the need for solutions onto other actors. Moser and Ekstrom note that barriers may be confounded with limits, but that they are not identical; a barrier is simply a limit that can potentially be overcome. I would argue that in remediation there are only barriers, not limits. For example, some might consider regulatory statutes a limit, but statutes and laws can be changed given sufficient political will and determination. Resources are not a limit; while finite, they can be reallocated, and/or used more efficiently and effectively. As a profession, medicine tends to focus on logistical challenges, conceiving them as limits but, as this thesis argues, instead of focusing solely on optimizing the logistics of remediation, or on identifying more resources, perhaps we first need to look at other variables. As Ostrom notes: "One can become fixated on a low conceptual hill by trying to optimize specific variables while overlooking better solutions involving ignored variables."11 This thesis has tried to identify ignored variables. While it has focused on the first phase of Moser and Ekstrom's framework (how we understand the problem) and on one particular stage in that phase (how we (re)define the problem) there are additional barriers beyond the obvious logistical and resource obstacles that our research suggested and that may be worth further study down the road. 
The first such barrier is remediation's toll on those who are organizing and implementing it. As noted in chapter 3, being involved in remediation of one's peers is 'emotionally and cognitively difficult', not only for those working one on one with remediatees, but perhaps even more so for those, such as department heads, who must inform individuals that they need to be remediated and also decide the details of that remediation. While remediating peers and feeling that one is benefiting both a fellow professional and the broader public can be deeply satisfying, it can also be quite stressful. This stress may arise not only from working with the occasional angry remediatee who lacks insight, but also from bearing witness to these same individuals coming to the realization and acceptance that their practice was not as good as they had believed it to be. A couple of our participants compared it to supporting a patient going through Elizabeth Kubler-Ross' five stages of dealing with grief: denial, anger, bargaining, depression, and acceptance. Perhaps we should say that remediation is not only related to education and regulation, but also connects with a distinctive type of peer support. The emotional challenge of working with remediatees, of walking that road with them, may be another barrier to involvement with remediation that requires further exploration. Several of our contacts for the remediator study noted how difficult it is to recruit remediators; whether a better understanding of the emotional challenges and better support would make a difference is unknown.

Another possible barrier is the potential role of the institution in which the physician works. Two of our department head participants mentioned that they sometimes worried that they had indirectly contributed to problems by either putting people in positions for which they were not suited, or by making unreasonable demands on individuals' time. In a similar vein, a health authority participant noted the role of the institution, and stated that problems "get swept under the carpet a little bit and it sort of kind of smolders along until someone decides, oh, this situation's intolerable. And then they just get all reactive and don't follow any process, you know, they fire the person or revoke privileges or what have you. And to me, that's just not a useful sort of structure or process to have." (data from the study presented in chapter 4, included in a presentation at AMEE 2017) The literature confirms that the institution in which the physician works is not always blameless. In fact, the workplace was identified as a contributing factor in over $80 \%$ of the cases seen by the United Kingdom's NCAS (National Clinical Assessment Service)..$^{12}$ However, unlike the individual physician, the institution was not obligated to accept NCAS' recommendations for change. One can imagine that this does not engender remediatee trust in the system or foster their engagement in the remediation process. Defining the problem as a problem of the individual rather than as a joint or systemic problem not only serves institutional interests by absolving them of the need to change, it is deeply imbedded in their individualistic culture.

We thus identified several barriers that go beyond the obvious ones of logistics. One logistical barrier that is rarely discussed but that bears mentioning here is the fact that remediation is not integrated into the CPD education system. The comment "It's just a capacity issue. No one has the time and the effort to put [in] to it" (chapter 4), would not make sense if remediation was considered an integral component of CPD; were it considered integral to CPD, some way would have to be found to commit the time and resources. Integrating remediation into CPD presents both logistical and conceptual challenges. 
One way remediation might be integrated into CPD would be to consider Ellaway et al's proposed zone-based model of remediation in training; the authors suggested that the principles of this model might apply to continuing medical education, although they did not elaborate on this. ${ }^{13}$ This model and its potential implications for remediation in CPD are discussed further below.

\section{Remediation in practice versus in residency}

As noted earlier, I began the $\mathrm{PhD}$ with the firm conviction that remediation in practice is somehow different from that in residency, and that this difference contributes to some of the specific challenges in addressing remediation at the practice level. In terms of the conduct of remediation, in many ways remediation at the practice level is not that different from remediation at the residency level. This was confirmed by a recent paper looking at the 'Dos, Don'ts and Don't Knows' of remediation in training which could, with minor modifications, have been written for remediation at the practice level. ${ }^{14}$ Chapter 5 did reveal some unexpected logistical differences. For example, patients never know when the resident responsible for their care is in a remedial rotation or program. They expect to see evidence of supervision. At the practice level, explanations can be tricky. Patients may even, as we heard in one case, feel that their physician, whom they trust, is being unfairly treated by the regulatory authority, and view the remediator as antagonistic to patients' best interests rather than an advocate for high quality patient care. However, in most cases, patients appeared to accept the explanations offered so that this was not a common problem. Generally, logistical issues - e.g. funding, covering for the remediating individual - are not that different across the continuum.

I had presumed that the major difference with remediation in practice was primarily related to remediating a peer. To be sure, there were particular problems associated with remediating a peer. The natural hierarchy between resident and faculty member, which helps mitigate potential power struggles at the training level, is absent or even inverted at the practice level. At the practice level, the fact that proportionally more remediatees are older men, and more remediators are younger women, a dual power inversion, can be hard to negotiate. The cultural background of some remediatees, where younger people are expected to defer to their elders, and women to men, can compound the problem. The loss of face inherent in requiring remediation adds fuel to the fire. More than one remediator participant suggested that gender and power issues needed to be discussed frankly up front, and that in some cases, it might be necessary to acknowledge that a male remediator might be more successful. This has not been discussed anywhere in the literature. We do know from the literature that students and residents rate male faculty members more favorably; however, faculties of medicine do not use that fact as a reason to preferentially choose male faculty..$^{15-17}$ If a remediatee has a higher chance of success with a male remediator, do we, in the interests of that individual's patients, accept being complicit with that remediatee's prejudices and biases by providing a male remediator? Or do we consider that by forcing the person to confront their sexism, we might also be helping their patients? The answer is neither easy, nor obvious. 
That said, the literature review and the research findings have led me to think that the difference has as much to do with the regulatory (i.e. loss of professional autonomy) aspect of remediation in practice as it does with remediating a peer. The regulatory aspect is what really complicates remediation in practice. While residents may not agree with the decision to invoke remediation, they generally do not contest the educational program's right to do so. Nor do they insist that their way of practicing is the correct one and that their peers are wrong. They do not criticize their training program for too much oversight and meddling into their affairs, or accuse them of being anti-doctor. Residents do not use their professional autonomy as a defense against requiring remediation.

To summarize, remediation in practice is complicated by the logistical, cultural and professional challenges inherent in a process that, temporarily at least, diminishes an individual's professional autonomy and thus may affect their professional and personal identity. This challenge may be contributing to the difficulty in recruiting remediators, as well as to remediatee response to being required to undergo remediation.

\section{Implications of remediation as both education and regulation}

Our data showed that remediation is conceptualized both as education and as a regulatory process. As became evident in our work, the duality becomes clearly problematic at the practice level because physicians, as professionals, are expected to both keep up to date and practice competently, as well as to have the autonomy to practice as they see fit. ${ }^{18,19}$ Reconciling the educational and regulatory aspects of remediation presents challenges. It is no coincidence that the North American jurisdiction that has developed, implemented and studied remediation processes systematically over two decades now is the Canadian province of Québec, a province with the distinction that the regulatory college is also the certifying body, and therefore responsible for the quality of medical education across the continuum. ${ }^{20,21}$ Almost two decades ago, it was deemed that the physician responsible for remediation in the Collège des Médecins (the regulatory body) must hold an advanced degree in education. While Québec's process is certainly not perfect, they appear to have broader acceptance for remediation and to be less challenged than other jurisdictions with, for example, recruiting remediators. The fact that education and regulation are both mandates of the regulatory authority appears to have played a role in Québec's relative success with remediation.

Since it is highly unlikely that other jurisdictions will be able to emulate Québec's distinctive system, we, might search for other ways to contend with the education/regulation duality. One possibility might be to modify Ellaway et al's 5-zone model mentioned earlier to apply at the CPD level. This model distinguishes between remedial action and remediation. At the training level, the 5 zones are intended to be part of a single model that integrates remediation into medical education. ${ }^{13}$ Zone 1 includes normal educational processes for those whose performance is at or above expected level. Successive zones from 2 through 5 represent increasing levels of remedial attention, from correction (what the authors call remedial action), through remediation and probation, to zone 5, exclusion. At the CPD level, this becomes more complicated, again because of the additional implications for 
professional autonomy. Their model would suggest that the educational component, which includes remedial action, lies in zones 1 and 2, and the regulatory component, which at the practice level would include remediation, practice limitation, and ultimately loss of licensure, lies in zones 3 and up. The challenge is this: while we might be able to distinguish between a remedial action (zone 2 correction) and remediation (e.g. a onehour coaching call might fall under 'remedial action' and supervised practice would be considered 'remediation'), since they are both mandated by the regulatory authority, it is unclear whether that distinction would make the remedial action more palatable and less stigmatizing than remediation. It might, however, be useful in resource planning and outcome reporting; some of the differences in reported success rates may be due to differences in the percentage of remedial action versus 'true' remediation.

The education/regulation duality isn't a priori problematic; it only becomes so when we don't acknowledge that both are operating at the same time i.e. when we don't take the implications of that duality into account when designing remedial interventions. I would argue that for remediation to be successful, all parties must acknowledge that remediation is both education and regulation, and explicitly discuss how these two aspects are to be managed. The need to discuss and negotiate power, particularly in the case of an older male remediatee with a younger female remediator, may be at the bottom an education vs regulation issue. The remediator wants to be an educator but the remediatee is focused on the regulatory implications and therefore resists the educational positioning.

\section{Remediation as a polarity to be managed}

My understanding of remediation evolved during the course of this thesis. I had been thinking about remediation as a generally wicked problem; now I realize more specifically the underlying nature of that 'wickedness' i.e. that remediation might be framed as a duality of both education AND regulation. Conceiving of remediation as a duality offers several options from which to choose when considering how to grapple with it. The first such option is to think of it as a paradox. Moroz states: "Framing competing demands as paradoxes offers a way of overcoming cognitive dissonance resulting from the need to engage both demands regardless of how contradictory they are, and often results in creative and novel approaches to achieving one's overarching goals."22 Another option would be to think of the remediation duality as a divergent problem. The economist E.F. Shumacher distinguished between convergent problems (those that can be solved) and divergent problems (those that must be transcended). ${ }^{23}$ An example of a divergent problem would be choosing between freedom and equality in society or between justice and mercy in dealing with bad behavior. Each seems to be the opposite of the other, but both are necessary. A third option would be to consider the business world's framing of dualities as polarities to be managed. ${ }^{24}$ Of the three - a paradox, a divergent problem, or a polarity - a polarity might be the most useful concept to apply to how we think about managing remediation's education vs regulation duality, since it is the only one that offers specific suggestions for managing that duality. 
Polarity theory suggests that dualities are not uncommon, and that rather than trying to determine which of two poles, in this case education or regulation, is the more important one, we should instead focus on both. It then provides us with tools to do just that. The education versus regulation aspect of remediation qualifies as a polarity because a) it describes an ongoing tension and b) the two poles, education and regulation, are interdependent i.e. focusing on one at the expense of the other leads to a new set of problems. ${ }^{24, \text { page }} 80$ (In contrast, university versus regulatory authority responsibility for developing remediation programs is not a polarity because a decision could be made that it should be one or the other and the 'problem' would be 'solved'.) According to polarity theory, the polarity cycle starts when we become overly focused on the negative aspects of one pole, and come to see the opposite pole as the solution to our problem. We then focus on that pole; however, after a while, the negative aspects of that side of the polarity become problematic, and we start seeing the opposite pole as the solution. We then cycle between poles in an infinity loop. If we take that concept and try to see how that might play out with regards to the education/remediation polarity, the story might go as follows.

Certain remediation stakeholders start to believe that too much emphasis is being placed on remediation as education, with the result that remediatees do not take the process seriously enough and that there have been complaints in the media that the profession is too focused on 'educating' dyscompetent doctors instead of mandating that they improve. These stakeholders successfully persuade their colleagues, and remediation programs then shift emphasis to focus on regulation. At first, this seems to have solved their concerns. Over time, however, several remediation stakeholders start noticing that while short-term outcomes have improved, long-term outcomes have worsened and that the stigma of remediation has increased with the result that physician resistance to the process has increased and engagement has decreased. Concerned stakeholders then argue that for remediation to be ultimately successful, it should be conceptualized as education, with the focus on preserving autonomy. These stakeholders are successful in their advocacy for change and remediation programs evolve to become all about education. At first, this appears to have solved the problem. A few years pass. Certain remediation stakeholders start to believe that too much emphasis is being placed on remediation as education, and the cycle begins anew thus continuing the infinity loop characteristic of poor polarity management.

A polarity map for the education vs regulation polarity might therefore look like the diagram below. The arrows demonstrate the infinity loop of cycling between poles that can occur if one is not aware of the positive and negative aspects of each pole. A wellmanaged polarity is one where we manage to stay mainly in the upper two quadrants of the polarity map i.e. where we can gain the benefits of both poles while avoiding as much as possible the downsides of each. In a poorly managed polarity, most of the time is spent either flipping between the downsides of both poles, or stuck in one downside. ${ }^{24}$ page 72 


\begin{tabular}{|c|c|c|}
\hline & EDUCATION & REGULATION \\
\hline $\begin{array}{l}\text { Upsides of focus on } \\
\text { this pole }\end{array}$ & $\begin{array}{l}\text { - Constructive, not punitive } \\
\text { - May decrease stigma } \\
\text { - May increase engagement } \\
\text { - Part of education system } \\
\text { - Focus on skills }\end{array}$ & $\begin{array}{l}\text { - Clarity re necessity for change } \\
\text { - Public reassurance }\end{array}$ \\
\hline $\begin{array}{l}\text { Downsides of focus on } \\
\text { this pole }\end{array}$ & $\begin{array}{l}\text { - De-emphasizes seriousness } \\
\text { - Emphasizes individual rather } \\
\text { than societal good }\end{array}$ & $\begin{array}{l}\text { - Ignores individual \& circumstances } \\
\text { - Decreases individual autonomy } \\
\text { - Focus is punitive, not constructive }\end{array}$ \\
\hline
\end{tabular}

If we wanted to skillfully manage the education/regulation polarity, we would appreciate that there are downsides to both poles, and watch for times when the downsides of either pole are becoming problematic. We would then move to the upper side of the opposite pole. ${ }^{24}$ page ${ }^{105}$ For example, if we noticed that remediatees did not appear to be taking the process seriously enough, we would stress the regulatory aspect of remediation and might ensure that a clear message was sent prior to the beginning of an individual remediation program. If we noticed that the felt loss of autonomy was interfering with remediatee engagement, we would find a means to increase that autonomy and help them focus on education, what one of our remediator participants described as helping people find joy and fulfilment in improving their practice. The goal is to maximize the advantages of both remediation as education and remediation as regulation, while avoiding or minimizing the disadvantages.

As noted earlier, polarity theory can help us in our conceptualization of remediation by suggesting that dualities are not uncommon, and that rather than trying to determine whether education or regulation is the more important aspect, we should instead focus on both. While this might seem at first glance to be impossible, we might start by acknowledging that there are positive and negative aspects to both remediation as education and remediation as regulation. Challenges and barriers might then be conceptualized as too much focus on one pole/not enough on another. For example, the tendency to 'other' remediatees could be conceptualized as arising from too heavy a focus on the regulatory aspect of remediation. An over focus on the regulatory aspect might also be seen to contribute to remediatee resistance and to hesitancy on the part of universities to get involved in remediation.

There appear to be fewer barriers related to an over focus on the educational aspect of remediation. One possible barrier is that remediators are so focused on the educational aspect that, while they may be to some extent conscious of the regulatory/loss of autonomy implications, they do not consider or address these in their dealings with the remediatee and thus miss an opportunity to defuse some of the tension inherent in having a peer oversee one's learning. The other barrier related to an over focus on the educational aspect is that is might potentially explain why regulatory authorities state that they are not educators and that universities should conduct remediation. However, I would argue that this is not a result of over focus on the educational aspect, but an attempt to mitigate 
the regulatory aspect of remediation. Perhaps we (the profession at large, remediation stakeholders in particular), while paying lip service to remediation's educational aspect, are in fact overly focused on the regulatory aspect. Or rather, by keeping the regulatory (loss of autonomy and therefore a temporary de- professionalization) aspect tacit, rather than explicit, we avoid difficult conversations with remediatees that might make the process easier in the long run.

\section{Reflexivity}

I began the PhD with several beliefs/assumptions: that barring serious health or cognitive issues, it is always worthwhile to attempt remediation; that we need to focus on prevention at the earliest possible level throughout a physician's practice career; and that remediation is everyone's problem - the regulatory authority's, the university's, the health authority's and the professional medical association's. I still maintain these beliefs, but I have become more aware of the underlying complexity of remediation. I have come to realize that simply trying to persuade various stakeholders that they should get involved will be insufficient to move remediation forward. I have noticed that some stakeholders tend to believe (I am tempted to say 'to wish') that if we can only catch people early enough in their downward drift into incompetence, we won't need formal remediation. This came out both in our interviews with stakeholders, and in a national remediation symposium that I attended in the fall of 2019. While I agree wholeheartedly with the importance of early diagnosis and intervention, there will always be those who'fall' rather than 'drift' into incompetence; even with the latter, the drift can be far advanced before the need for a course correction appears. This wish that the need for remediation would simply disappear is something I had not fully realized when I began my research. I now wonder whether the wish/belief that sufficiently robust prevention strategies will preclude the need for remediation may also serve to justify avoiding investment in remediation programs.

\section{Implications}

I now ask myself: were I the director of the same remediation program today as I was 12 years ago, i.e. running an assessment and remediation program out of a university CPD office, what would I do differently taking into account what I have learned in the course of this PhD? Or, the more likely scenario, were I asked to offer advice to today's leaders of remediation programs, what would I tell them? To start, I would want to discuss the education/regulation polarity and the importance of trying to remain in the upper quadrants of the polarity map. I would need to be clear what was meant by regulation, and how remediation could be perceived/conceptualized as a loss of self-regulation and a de facto (temporary) de-professionalization (chapter 3). I would suggest that they need to openly discuss both the ambiguity and the ambivalence surrounding remediation with their various stakeholders, and I would reassure them that many of these stakeholders would not be surprised, since these concepts came about by interviews with either them or their colleagues. I would suggest that they use those concepts when attempting to persuade the medical associations and the various university department heads that they 
have a moral obligation to the profession, to the individual physician and to the public, to get involved with remediation.

I would suggest to these remediation program developers that they also engage remediatees in the construction of remediation plans that provide them with as much autonomy as possible. If remediation can be construed as a de-professionalization, with its implied challenge to professional identity, then allowing remediatees to preserve as much of that autonomy as possible should facilitate their engagement in the process. Along those lines, I would suggest to regulatory authorities that it might be interesting to see what happens when conversations with remediatees start along these lines: "It appears that you need to make certain changes to your practice. For some reason, you have been unable to identify or to make those changes on your own, so as the organization responsible for patient safety and quality of care, we are obligated to take on the responsibility to oversee those changes and to ensure that they happen. This is what we propose to do: we will.....you will.........We realize that this is difficult for you, that we are temporarily taking away part of your professional autonomy; if there is anything we can do to make this easier for you without compromising either the process or the desired outcome, we would be happy to discuss it. Some physicians have told us that in retrospect they are actually happy that they had to undergo remediation (see chapter 5) and that it was helpful in many ways. We would suggest that you think of this as an opportunity to access the best continuing medical education you will ever get: one-on-one coaching to help you take care of your patients better [see chapter 3], and perhaps even make your practice more efficient. We will also work with you to develop processes you can follow going forward so that you don't end up in this situation again." Might being explicit about the duality augment the upsides of remediation's two poles of education and regulation and diminish the downsides?

As another way to facilitate engagement, I would strongly encourage program developers to ensure that the remediator and the person assessing the success of the remediation were two different individuals. If, as we saw from our interviews with preceptors, (chapter 5) being both educator and judge can be problematic, it would make sense to separate those two roles. That separation has been found to be useful in another context i.e. IMG supervision. Several Canadian provinces have programs whereby international medical graduates (IMGs) are allowed to practice under remote supervision after an initial assessment period. These IMGs also have a mentor, but the mentor role has been purposely designed to be separate from the supervisor role so that the IMG can discuss areas of uncertainty without worrying that these will get back to the regulatory authority and negatively influence perceptions of their competence. A trusted mentor might similarly encourage remediatees to openly discuss areas they might improve.

One of the biggest challenges for remediation program directors, according to those through whom we went to access participants for our remediator study, is recruiting preceptors. I would suggest to these directors that one way to address that concern might be by providing faculty development for remediators that addressed the concerns identified by our remediator participants, as well as discussing how to identify the positions of educator, judge and public defender, and how to move gracefully between 
them as needed. I would also develop and support a community of practice (in the sense of cultivating a learning group of remediators) and advocate for their work to be understood as the important contribution to the community that it is; this includes advocating for this work to be considered in tenure decisions ${ }^{25} \mathrm{~A}$ community of practice would have several benefits. More experienced remediators could mentor newer ones in a way that a program director not directly remediating cannot. One of our novice remediator participants noted that she wished she could have observed a more experienced remediator handle a particularly difficult situation. A CoP might facilitate that; at the very least, it would allow group discussion of common experience and tactics. The community could advocate for the faculty development and recognition it needs. It could also provide insights into the local situation that would enable better local program development.

Finally, I would endeavor to perform the above in a scholarly manner and to share what was learned with the wider community through conferences and publications. As was noted in chapter 2, much of what is available regarding remediation in practice, particularly data on programs and outcomes, is never published, or rarely, in the grey literature. Much of it is either kept internally or shared at conferences and meetings. As a remediation program director, I would now advocate for funding to hire a researcher.

\section{Future research}

The one group notably absent from this program of research are the remediatees. Gaining access to that group will present both logistical and ethical considerations, since the individuals with access to remediatees are the regulators, who will be deemed to have undue influence/power to persuade them to participate. A recent Dutch paper describes a study interviewing physicians who had been disciplined for a variety of offenses including substandard care and inappropriate behavior towards patients; however aspects of their protocol e.g. "professionals that did not respond were phoned up to five times as a reminder" would not be acceptable to a Canadian ethics review board. ${ }^{26}$ We have begun conversations with our ethics review board to determine how we can address their concerns. It will be important to interview remediatees to not only understand how they, as the target of remediation, conceptualize the process, but also to understand the impact on their professional and personal identity. Taken together, this might also potentially help us gain insights into how to better structure remediation.

The words we use - remediation, clinical supervision, preceptor, supervisor, etc. - affect how we and others conceptualize remediation, and in all likelihood also how remediation is experienced. A discourse analysis of remediation that included documents on regulatory and university websites along with the data from our studies might provide further insight into how we conceptualize remediation. It might also illuminate the power challenges in remediation which were noted in chapter 5 .

Our results also suggest several remediation topics other than conceptualizations that might be fruitful grounds for research. For example, what is the role of insight in remediation? Several respondents in our stakeholder study mentioned that remediatees 
frequently lack insight, but what level of insight is necessary for successful remediation? How do we foster insight without devastating the individual i.e. how do we support someone through the process of coming to terms with their need for remediation? The many aspects of insight in remediation would provide material for another PhD.

Another fruitful area for research might be whether remediation, and the events that lead to its invocation, end up being associated with shame or guilt. The 'not-like-us' that some of our study participants described, and the 'maybe those people don't have it in them to be what they need to be' suggests that competence, and the capacity to maintain it, is sometimes considered a trait, not a state. This would lead to shaming of those who aren't fully competent, and remediatees feeling shame at needing remediation. Might the selfconscious emotions (e.g. feeling shame and therefore avoiding showing one's ignorance by seeking help) play a role both in the need for remediation and the challenges associated with remediation for remediatees? ${ }^{27}$

As mentioned earlier, the Québec program appears to function most effectively, in that it has consistently for over two decades studied and refined its processes, engaged university and community partners, and disseminated its results in the literature and at international meetings. Informally, the Québec program has identified several questions that would benefit from structured study. For example, they have noted that younger physicians are more successfully remediated than their older counterparts; they question whether this is due to a difference in motivation - older physicians closer to retirement may not be as motivated as physicians earlier in their career - rather than a difference in age-related learning ability but don't have an answer (personal communication, François Goulet). The program has also identified a cohort of physicians who appear to be successfully remediated in that they meet their learning objectives but whose practice continues to be substandard on reassessment; the question is whether these physicians are unable to transfer learning or whether other factors are at play (ibid). If we can access some of these physicians in our remediatee study, we may hopefully gain insights into some of these questions.

Finally, the factors identified at the beginning of this $\mathrm{PhD}$ as contributing to increasing discussions on remediation continue to play a role, as evidenced by the increasing number of papers on remediation in the intervening few years. The majority of these papers have been at the training level, with a couple of conceptual pieces that might also have applications at the practice level. ${ }^{13,14,28-34}$ Papers at the practice level suggest that in spite of new programs ${ }^{34}$, challenges abound ${ }^{12}$ and best practices remain elusive $\mathrm{e}^{35,36}$ while the need to determine these only grows. ${ }^{9,12,35-37}$ There are thus many areas around remediation in practice for researchers to consider.

\section{Conclusion}

This program of research has explored how the medical profession conceptualizes remediation in practicing physicians and those individuals who require it, with a focus on the remediation of clinical competence gaps. We found that remediation is both education 
and regulation; the failure to identity and/or acknowledge that duality can explain some of the barriers to institutional involvement in remediation. It also contributes to some of the challenges experienced by remediators who work directly with physicians. We identified ambivalence in regards to physicians requiring remediation who are viewed as both one-of-us and as 'other'; this ambivalence also contributes to the hesitancy to both personal and institutional involvement in remediation. Together, these help explain some of the challenges the medical profession has had in developing programs and processes to address the remediation of clinical competence gaps in practicing physicians. 


\section{References}

1. Collins ME, Block SD, Arnold RM, Christakis NA. On the prospects for a blame-free medical culture. Social science \& medicine (1982). 2009;69 (9):287-1290.

2. Tezuka K. Physicians and professional autonomy. Japan Medical Association Journal 2014;57 (3) 154-158.

3. Ross L. The intuitive psychologist and his shortcomings: Distortions in the attribution process. Advances in Experimental Social Psychology 1977;10:173-220.

4. Lerner MJ, Miller DT. Just world research and the attribution process: Looking back and ahead. Psychological Bulletin 1978;85 (5):1030-1051.

5. Canales MK, Canales MK. Othering:Toward an Understanding of Difference. Advances in Nursing Science 2000;22 (4)16-31.

6. Goffman E. Stigma. Simon and Shuster; 1963.

7. Hodges B. Medical education and the maintenance of incompetence. Medical Teacher 2006;28 (8):690-696.

8. Lingard L. Rethinking competence in the context of teamwork. The question of competence: Reconsidering medical education in the twenty-first century. 2012:42-69.

9. Goulet F, Thiffault J, Ladouceur R. Remediation and rehabilitation programmes for health professionals: challenges for the future. BMJ Quality \& Safety 2017;26 (12):941-943.

10. Moser SC, Ekstrom JA. A framework to diagnose barriers to climate change adaptation. Proceedings of the National Academy of Science. 2010; 107 (51):22026-22031.

11. Ostrom E. A diagnostic approach for going beyond panaceas. Procedings of the National Academy of Sciemces U S A. 2007;104 (39):15181-15187.

12. Price T, Archer J. UK Policy on Doctor Remediation: Trajectories and Challenges. Journal of Continuing Education in the Health Professions 2017;37 (3):207-211.

13. Ellaway $\mathrm{RH}$, Chou $\mathrm{CL}$, Kalet AL. Situating remediation: accommodating success and failure in medical education systems. Academic Medicine 2018;93 (3):391-398.

14. Chou CL, Kalet A, Costa MJ, Cleland J, Winston K. Guidelines: The dos, don'ts and don't knows of remediation in medical education. Perspectives on Medical Education 2019;12 (8):322-228.

15. Morgan HK, Purkiss JA, Porter AC, et al. Student Evaluation of Faculty Physicians: Gender Differences in Teaching Evaluations. Journal of Women's Health 2016;25 (5):453-456.

16. Fassiotto M, Li J, Maldonado Y, Kothary N. Female Surgeons as Counter Stereotype: The Impact of Gender Perceptions on Trainee Evaluations of Physician Faculty. Journal of Surgical Education 2018;75 (5):1140-1148.

17. MacNell L, Driscoll A, Hunt AN. What's in a name: Exposing gender bias in student ratings of teaching. Innovative Higher Education 2015;40 (4):291-303.

18. Armstrong D. Clinical autonomy, individual and collective: the problem of changing doctors' behaviour. Social Science \& Medicine 2002;55 (10):1771-1777.

19. Friedson E. The Characteristics of a Profession. In: Earle SL, G. , ed. The Sociology of Health Care: $A$ reader for Health Professionals. UK: Palgrave Macmillan; 2008.

20. Goulet F, Gagnon R, Gingras ME. Influence of remedial professional development programs for poorly performing physicians. The Journal of Continuing Education in the Health Professions 2007;27 (1):42-48.

21. Goulet F, Jacques A, Gagnon R. An innovative approach to remedial continuing medical education, 1992-2002. Academic Medicine 2005;80 (6):533-540. 
22. Moroz A. 3AC: dealing with tensions in assessment (and elsewhere). Medical Education. 2019;53 (1):8-10.

23. Schumacher EF. Small is beautiful: A study of economics as if people mattered. London: Sphere Books Ltd 1974.

24. Johnson B. Polarity management: Identifying and managing unsolvable problems. Human Resource Development; 1992.

25. Buckley H. When I say ... community of practice. Medical education 2019;53 (8):763-765.

26. Verhoef LM, Weenink J-W, Winters S, Robben PBM, Westert GP, Kool RB. The disciplined healthcare professional: a qualitative interview study on the impact of the disciplinary process and imposed measures in the Netherlands. BMJ Open. 2015;5 (11):e009275.

27. Bynum WE, Artino AR. Who am I, and who do I strive to be? Applying a theory of self-conscious emotions to medical education. Academic Medicine 2018;93 (6):874-80.

28. Skelton JR, Wiskin CM, Ward JDT. Understanding professional development: Case studies of remedial support. Medical Teacher 2019;41 (12):1-8.

29. Lacasse $M$, Audétat $M-C$, Boileau É, et al. Interventions for undergraduate and postgraduate medical learners with academic difficulties: A BEME systematic review: BEME guide no. 56. Medical Teacher 2019;41 (9):981-1001.

30. Hu WC, Woodward-Kron R, Flynn E. Educator as Diagnostician, Judge and Confidant: a positioning analysis of medical student support encounters. Advances in Health Sciences Education 2019;24 (4):702-724.

31. Shearer C, Bosma M, Bergin F, Sargeant J, Warren A. Remediation in Canadian medical residency programs: Established and emerging best practices. Medical Teacher 2019;41 (1):28- 35.

32. Yepes-Rios M, Dudek N, Duboyce R, Curtis J, Allard RJ, Varpio L. The failure to fail underperforming trainees in health professions education: A BEME systematic review: BEME Guide No. 42. Medical Teacher 2016;38 (11):1092-1099.

33. Krzyzaniak SM. A qualitative study of medical educators' perspectives on remediation: Adopting a holistic approach to struggling residents. Medical Teacher 2017;39 (9):967-974.

34. Kalet A, Guerrasio J, Chou CL. Twelve tips for developing and maintaining a remediation program in medical education. Medical Teacher 2016;38 (8):787-792.

35. Prescott-Clements L. Rethinking Remediation: A Model to Support the Detailed Diagnosis of Clinicians' Performance Problems and the Development of Effective Remediation Plans. The Journal of Continuing Education in the Health Professions 2017;37 (4):245-254.

36. Weenink J-W. Getting back on track: a systematic review of the outcomes of remediation and rehabilitation programmes for healthcare professionals with performance concerns. $B M J$ Quality \& Safety 2017;26 (12):1004-1014.

37. Brennan N. Remediating professionalism lapses in medical students and doctors: A systematic review. Medical Education 2019; December 23. Published ahead of print. 

Summary 

The need to develop remediation programs for practicing physicians has never been greater. Increasing public demands for physician accountability and the ensuing development in several countries of revalidation processes and/or practice improvement programs are the main impetus for this state of affairs, abetted by high profile cases such as Shipman, the UK physician who was also a serial murderer. While it might be reasonably expected to be easier to remediate deficiencies in clinical competence than professionalism gaps or mental health and addictions issues negatively affecting performance, until now there have been more programs to support physicians struggling with mental health and addictions than to address gaps in clinical competence. This thesis explores possible reasons for this state of affairs. Since the first step in solving a problem is in defining it, the focus of this thesis is on how remediation in practicing physicians is conceptualized, and on how these conceptualizations might be contributing to the current situation.

Chapter 1 explains the background of this thesis and how personal experience as the director of an assessment and remediation program for practicing physicians led me to want to do a $\mathrm{PhD}$ to explore why remediation in practice is so fraught. I found it particularly telling that a paper written more than 30 years ago asking questions such as where remediation fits into the CME/CPD educational system, who pays for it and who provides it, could have been written today. Based on the literature and on personal experience as a member of international organizations of remediation programs, this challenge in addressing remediation appears to be consistent across countries, or at least across English-speaking countries, irrespective of the medical (state-funded vs private) or social systems. The lack of an agreed-upon definition for remediation and of what the term includes contributes to that challenge. It leads to widely varying estimates of the need for remediation ( $0.5 \%$ to $30 \%$ of the physician population), as well as to difficulty in interpreting what little outcome data we have. Canada has been a leader in the field of physician assessment and remediation and would thus appear to be a fruitful context in which to study remediation.

Chapter 2 consists of a scoping review of the literature on the remediation of practicing physicians, focusing on what is revealed explicitly or what might be revealed implicitly about the meaning of remediation for practicing physicians. The review includes the gray literature on remediation programs in North America and the United Kingdom, as well as other literature that contributes to our understanding of remediation, such as learning theories and theories on the sociology of medicine. This review suggests that remediation is conceptualized as an educational process, with the final aim of returning the individual to self-regulated, autonomous practice. Consistent with that educational perspective, remediation in the literature appears to be conceptualized as similar to remediation in residency with similar methods employed and similar remediator attributes and skills required. In regards to the individual requiring remediation (the remediatee), the literature focuses on understanding personal risk factors for poor practice. This focus seems to be more in the service of identifying these individuals so that their practice can be assessed, than in service of determining how to support at risk individuals before problems develop. Finally, the literature on medical culture suggests that aspects of that culture, including shaming and blaming individuals, the reluctance to challenge a colleague's practice, and the concept of professional autonomy might all affect to a greater or lesser degree 
the willingness to remediate. The conclusion of the scoping review was to suggest that a different way of looking at remediation - as supported practice change - and taking into account the social determinants of competence rather than focusing on remediatee characteristics might enable us to ask better questions. Finally, since culture is notoriously difficult to change, rather than wait for culture change to facilitate program development, it might be less complicated to normalize remediation, and thus change the culture, by developing widespread remediation programs.

Chapter 3 explores how representatives of different Canadian remediation stakeholder groups (certifying bodies, regulatory authorities, health authorities, university postgraduate and continuing professional development associate deans) grapple with the issue of remediation, and their underlying beliefs and assumptions regarding it. Data from one-hour semi-structured interviews were analyzed using constructivist grounded theory. Results showed that remediation stakeholders conceptualize remediation as having both educational and regulatory aspects and that they slip unconsciously between these two conceptualizations. [Regulatory here refers to the loss of self-regulation and of professional autonomy implied when an external agency removes an individual physician's decisions regarding their continuing education and/or practice.] This may help explain why universities hesitate to accept remediation as part of their mandate: they are focusing on remediation's regulatory aspect. On the other hand, regulatory authorities, those responsible for public protection, deem their main role to be assessment and consider remediation to be the purview of educators, i.e. of the university. Failure to come to grips with the education/regulation duality not only makes organizing remediation more difficult, it also leads to practice improvement initiatives being misconstrued as regulatory by the general physician workforce.

Chapter 4 utilizes data from the previously described semi-structured interviews to explore how the representatives from various stakeholder groups conceptualize individuals requiring remediation. Interactions with colleagues over the years lead us to believe that, in spite of the common public perception that doctors 'stick together', physicians may not be as supportive of their struggling colleagues as they could be. This was confirmed when stakeholders sometimes demonstrated ambivalence towards their struggling colleagues and about whether remediation, versus removal of licensure, is a wise use of scarce resources. Our participants simultaneously view competence gaps as a state, a place any physician can find themselves in if they are careless or unlucky, and as a trait, a characteristic of the individual suggesting they do not belong in the medical fraternity. This latter tendency to 'other' struggling colleagues can be explained by both various psychological theories and by the medical culture of autonomy where the individual is solely responsible for developing and maintaining competence.

Chapter 5 explores the remediators' (those who are asked by regulatory and health authorities to work one on one with physicians with significant competence gaps and oversee their learning and practice) underlying conceptual frameworks around remediation for practicing physicians, their motivation for being involved in this activity, and how they view the physicians they remediate. Asking remediatees for stories of particularly memorable remediation experiences enabled further exploration of the 
phenomenon of remediation without attaching any preconceptions as to what might be found. An analysis of their narratives using positioning theory as a sensitizing concept revealed that remediators move between the positions of educator, judge, and guardian, and that while educator is their preferred position, regulators and remediatees might inadvertently or deliberately place obstacles in their way. This suggests that failure to acknowledge the education/regulation duality and to train remediators to identify and move between different positions in the remediator role may be contributing to making the remediation experience more challenging for remediators than it needs to be.

Chapter 6 This commentary on a paper regarding the do's and don'ts of remediation, written at the end of the PhD and thus informed by our research findings, posits that if we don't change medicine's culture of independence and individual self-regulation, remediation will continue to be problematic no matter what we call it or how we organize it.

Chapter 7 This thesis has attempted to understand barriers to organizational (university, health authority, medical association, regulatory body) involvement with remediation. Whereas the literature and conversations at meetings focus on logistics, I explored less tangible barriers such how we conceptualize remediation and those who require it. This final chapter discusses how this program of research has refined and shaped my conceptions of the nature of remediation for practicing physicians, and where those new conceptions might lead to. Using the lens of polarity theory, remediation might now be viewed as a duality with two apparently contradictory poles, education and regulation, that can and must be managed. Keeping the two poles in balance, maximizing the upsides of both poles and minimizing the downsides, might help us move forward with the development of remediation programs as an integral component of the continuing professional development. At the same time, we must address 'othering' of struggling physicians, either by stressing that given the right circumstances any physician could find themselves in a similar situation, or by encouraging a shift from exclusionary to inclusionary othering. The first step may be to speak with physicians who have undergone remediation in order to understand how they conceptualize the process and experience its impact. 

Samenvatting 

De noodzaak om remediëringsprogramma's voor praktiserend artsen te ontwikkelen is groter dan ooit tevoren. De belangrijkste aanleiding voor deze stand van zaken is de toenemende publieke vraag naar verantwoording door artsen en de daaruit voortvloeiende ontwikkeling van herregistratieprocessen en/of programma's voor praktijkverbetering in diverse landen, aangewakkerd door spraakmakende zaken zoals die van Shipman, de Britse arts die ook een seriemoordenaar was. Hoewel redelijkerwijs kan worden verwacht dat het wegwerken van deficiënties in klinische competentie eenvoudiger is dan het verhelpen van professionaliteitshiaten of problemen met de mentale gezondheid of verslavingen die de prestaties negatief beïnvloeden, bieden de tot nu toe in het leven geroepen programma's vooral ondersteuning aan artsen die worstelen met hun mentale gezondheid en verslavingen dan dat deze de hiaten in klinische competentie aanpakken. Dit proefschrift onderzoekt de mogelijke redenen voor deze stand van zaken. Aangezien het definiëren van een probleem de eerste stap is in de oplossing ervan, zal dit proefschrift zich richten op de vraag hoe remediëring bij praktiserend artsen wordt geconceptualiseerd en hoe deze conceptualiseringen mogelijk bijdragen aan de huidige situatie.

Hoofdstuk 1 licht de achtergrond van dit proefschrift toe, evenals hoe mijn persoonlijke ervaring als directeur van een toets- en remediëringsprogramma voor praktiserend artsen mij ertoe gebracht heeft om promotieonderzoek te willen doen naar de vraag waarom remediëring in de praktijk zo lastig is. Ik vond het bijzonder treffend dat een artikel dat meer dan 30 jaar geleden is geschreven en waarin vragen werden gesteld als "waar past remediëring in het medische bij- en nascholingssysteem?", "wie betaalt ervoor en wie biedt het aan?" vandaag geschreven had kunnen zijn. Afgaande op de literatuur en op mijn persoonlijke ervaring als lid van internationale remediëringsprogrammaorganisaties, blijkt het aanpakken van remediëring voor veel landen een uitdaging te zijn, althans voor Engelstalige landen, ongeacht hun medische (door de overheid gefinancierd vs. particulier) of sociale stelsels. Het ontbreken van een gemeenschappelijke definitie van remediëring en van wat daar allemaal onder valt maakt deze uitdaging alleen maar groter. Het leidt tot zeer uiteenlopende schattingen van de behoefte aan remediëring $(0,5 \%$ tot $30 \%$ van de artsenpopulatie), alsook tot problemen met het interpreteren van het beetje dat we hebben aan uitkomstdata. Canada is leider op het gebied van toetsing en remediëring van artsen en lijkt derhalve een zinvolle context om remediëring in te onderzoeken.

Hoofdstuk 2 bestaat uit een verkennende studie (scoping review) van de literatuur over remediëring van praktiserend artsen, waarbij de focus ligt op expliciete en impliciete vermeldingen van wat remediëring voor praktiserend artsen inhoudt. Deze literatuurstudie omvat de grijze literatuur over remediëringsprogramma's in NoordAmerika en het Verenigd Koninkrijk, alsook andere literatuur die bijdraagt aan onze begripsvorming van remediëring, zoals leertheorieën en theorieën over medische sociologie. Deze studie maakt aannemelijk dat remediëring wordt geconceptualiseerd als een educatief proces, met als uiteindelijke doel het individu weer te laten terugkeren in de zelfregulerende, zelfstandige praktijk. In overeenstemming met die educatieve zienswijze lijkt remediëring in de literatuur net zo geconceptualiseerd te worden als remediëring in de vervolgopleiding. Daarbij worden bovendien dezelfde methoden aangewend 
en zijn dezelfde eigenschappen en vaardigheden vereist voor de "remedieerder". Met betrekking tot het individu dat remediëring nodig heeft, richt de literatuur zich op het begrijpen van persoonlijke risicofactoren voor onprofessioneel handelen. Deze focus lijkt eerder te dienen om deze individuen op te sporen opdat hun praktijk kan worden beoordeeld, dan om te bepalen hoe deze risicopersonen kunnen worden ondersteund vóórdat zich problemen voordoen. Ten slotte suggereert de literatuur over de medische cultuur dat elementen uit die cultuur, waaronder het aanwijzen van een schuldige, de terughoudendheid om de praktijk van een collega ter discussie te stellen, en het concept "professionele autonomie", allemaal in meer of mindere mate de bereidheid tot remediëring zouden kunnen beïnvloeden. De conclusie van de scoping review was dat wij onszelf mogelijk betere vragen zouden stellen als we remediëring anders gaan zien, namelijk als begeleide praktijkverandering, en als wij sociale factoren die iemands competentie bepalen in acht nemen in plaats van ons te richten op eigenschappen van te remediëren individuen. Tot slot, aangezien cultuur notoir moeilijk te veranderen is, zou het wellicht minder ingewikkeld zijn om remediëring te normaliseren, en zodoende de cultuur te veranderen, door breed opgezette remediëringsprogramma's te ontwikkelen, in plaats van te wachten tot cultuurverandering intreedt die de ontwikkeling van dergelijke programma's mogelijk maakt.

Hoofdstuk 3 onderzoekt hoe vertegenwoordigers van verschillende Canadese groepen belanghebbenden op het gebied van remediëring (certificerende instellingen, regelgevende instanties, gezondheidsautoriteiten, vice-decanen van vervolgopleidingen en nascholingsprogramma's) worstelen met remediëring en wat hun onderliggende overtuigingen en uitgangspunten daaromtrent zijn. Data van één uur durende semigestructureerde interviews werden geanalyseerd met behulp van constructivist grounded theory methode. De resultaten toonden aan dat de genoemde belanghebbenden remediëring opvatten als een concept waaraan zowel educatieve als regulerende aspecten kleven en dat zij onbewust tussen deze twee conceptualiseringen in glippen ["regulerend" betekent hier het verlies van zelfregulatie en van de professionele autonomie zoals bedoeld wanneer een externe instantie aan een individuele arts de vrijheid ontneemt besluiten omtrent zijn/haar nascholing en/of praktijk te nemen]. Dit zou mede kunnen verklaren waarom universiteiten er niet happig op zijn om remediëring tot hun verantwoordelijkheden te rekenen: zij zijn te gefocust op het regulerende aspect van remediëring. Regelgevende instanties, daarentegen, dus diegenen gemoeid met het beschermen van de samenleving, beschouwen toetsing als hun voornaamste rol en zien remediëring als een taak die opleiders, d.w.z. de universiteit, toebehoort. Het feit dat men er niet in slaagt om uit deze onderwijs/regelgeving-dualiteit te komen, maakt remediëring niet alleen lastiger te organiseren, maar zorgt er ook voor dat praktijkverbeteringsinitiatieven in het algemeen verkeerd, namelijk als regulerend, worden opgevat door praktiserend artsen.

In Hoofdstuk 4 werd gebruik gemaakt van de data uit de eerder beschreven semigestructureerde interviews om na te gaan hoe de vertegenwoordigers van verschillende groepen belanghebbenden individuen die remediëring nodig hebben, conceptualiseerden. Contact met collega's door de jaren heen gaf aan dat, ondanks het algemene beeld dat men heeft dat artsen "elkaar de rug dekken", artsen wel eens niet 
zo solidair richting hun worstelende collega's zouden zijn als ze zouden kúnnen zijn. Dit werd bevestigd toen belanghebbenden soms ambivalentie vertoonden richting hun worstelende collega's en zich afvroegen of remediëring, tegenover het intrekken van de artsbevoegdheid, een verstandig gebruik is van schaarse middelen. Tegelijkertijd zagen onze deelnemers competentiehiaten als een toestand, een plaats waarin elke arts terecht zou kunnen komen als ze onverschillig waren of pech hadden, en als een eigenschap, een persoonlijke karaktertrek die aangaf dat ze niet in de medische stand thuishoorden. Deze laatste neiging tot het buitensluiten (othering) van worstelende collega's kan door verschillende psychologische theorieën worden verklaard, maar ook door de medische cultuur die autonomie vooropstelt, waarbij het individu als enige verantwoordelijk is voor de ontwikkeling en het behoud van competentie.

Hoofdstuk 5 onderzocht vanuit welke onderliggende denkkaders rondom remediëring voor praktiserend artsen remedieerders (degenen die door regelgevende instanties en gezondheidsautoriteiten gevraagd worden om één-op-één met artsen waarbij significante competentiehiaten zijn geconstateerd, te werken en hun leren en praktijk te overzien) handelen, wat hun motivatie is om bij deze activiteit betrokken te zijn, en hoe zij de artsen die zij remediëren, zien. Door de "te remediëren artsen" te vragen naar verhalen over bijzonder gedenkwaardige ervaringen met remediëring konden wij het fenomeen "remediëring" nader onderzoeken zonder daarbij vooraf al ideeën te hebben gevormd van wat er mogelijk zou kunnen worden gevonden. Een analyse van hun verhalen waarbij positioneringstheorie werd gebruikt als richtinggevend of sensitizing concept, onthulde dat remedieerders zich tussen de rol van opleider, beoordelaar en hoeder heen bewegen, en dat terwijl zij het liefst gewoon opleider hadden willen zijn; toch kunnen regelgevers en "te remediëren artsen" onbedoeld dan wel opzettelijk obstakels op hun pad plaatsen. Dit suggereert dat het miskennen van de onderwijs/regelgeving-dualiteit en het feit dat remedieerders niet worden getraind om een onderscheid te maken tussen de verschillende remedieerder-rollen en zich daartussen te bewegen, de ervaring met remediering mogelijk nog lastiger maken voor remedieerders dan dat nodig is.

Hoofdstuk 6. In dit commentaar op een artikel over de do's-and-don'ts van remediëring dat aan het einde van het promotietraject is geschreven en zich zodoende stoelt op onze onderzoeksbevindingen, wordt gesteld dat remediëring, hoe we het ook noemen of hoe we het ook organiseren, problematisch zal blijven als we de medische cultuur van onafhankelijkheid en individuele zelfregulering niet veranderen.

Hoofdstuk 7. Dit proefschrift heeft getracht inzicht te verschaffen in de factoren die organisaties (universiteiten, gezondheidszorgautoriteiten, artsenorganisaties, regelgevende instanties) ervan weerhouden bij remediëring betrokken te zijn. Terwijl de literatuur en gesprekken tijdens vergaderingen gericht zijn op logistieke knelpunten, ging ik op zoek naar minder grijpbare belemmeringen, zoals hoe we remediëring en degenen die remediëring nodig hebben, conceptualiseren. Dit laatste hoofdstuk bespreekt hoe dit onderzoeksprogramma mijn begrip van de aard van remediëring voor praktiserend artsen heeft verfijnd en gevormd en waar dit nieuwe begrip toe zou kunnen leiden. Door remediëring door de lens van polariteitstheorie te bekijken, zou remediëring nu kunnen worden opgevat als een dualiteit met twee ogenschijnlijk tegenstrijdige uiteinden, 
namelijk onderwijs en regelgeving, die kunnen en moeten worden afgestemd. Door de twee uiteinden in balans te houden, de voordelen van beide uiteinden maximaal te benutten en de nadelen te minimaliseren, zouden we in staat moeten zijn om remediëringsprogramma's verder te ontwikkelen als integraal onderdeel van nascholing voor artsen. Tegelijkertijd moeten we iets doen aan het voornoemde othering van worstelende artsen, zij het door te benadrukken dat menig arts zich in dezelfde situatie zou kunnen bevinden mits de omstandigheden er naar waren, zij het door aan te sturen op een verschuiving van buitensluitend naar includerend othering. De eerste stap zou kunnen zijn om met artsen die remediëring hebben ondergaan te praten teneinde inzichtelijk te maken hoe zij het proces conceptualiseren en het effect ervan ervaren. 
Addendum: Valorisation paragraph 

Increasing demands for physician accountability and quality improvement initiatives in healthcare are leading to more discussions regarding how best to address identified deficiencies in the care provided by individual physicians. Remediation programs struggle with logistics of how best to organize, implement, and fund programs. As someone who was for 10 years the director of such a program, I am well aware of the challenges involved. However, as my supervisors stressed at the beginning of the $\mathrm{PhD}$ process, the goal of a $\mathrm{PhD}$ is not to solve a problem; problems not only differ from place to place depending on the local context, but also have a way of morphing over time, so that solutions tend to be short-lived and only applicable to restricted contexts. The goal of a PhD then is not to solve a problem but to help people think about a problem differently, so that they can develop better solutions suited to their particular place and time. The goal of this PhD was thus not to determine the 'best' way to design and implement remediation programs for practicing physicians but to explore how the profession conceptualizes remediation and those requiring it, and to consider how these conceptualizations influence organizational willingness and ability to develop remediation programs for practicing physicians.

Valorisation in this case, then, is not so much a matter of developing new products or processes as it is of presenting my findings to those in a position to implement new processes such as organizations and institutions developing remediation programs. I was able to do so in an international venue at a meeting of regulators in June 2019, as well as at a national remediation symposium in September 2019. As a plenary speaker at the latter meeting, I was asked to discuss where I thought remediation in Canada should go. I was able to both present my research findings and suggest implications for change arising from my research. Several of the participants at that symposium had been interviewed for my research, and were thus particularly interested and attentive.

Idea that were shared and that resonated included our finding that remediation is both educational and regulatory (in the sense that it removes an individual professional's right to self-regulate both their learning and their practice). That finding might help explain why regulatory authorities would prefer universities to take on remediation programs - they are focusing on the educational aspect that universities are better equipped to provide. It also explains why conversely universities hesitate to become involved in remediation- they are focusing on the regulatory aspect, and don't wish to be viewed as an arm of the regulator. Finding ways to minimize the loss of self-regulation, perhaps by allowing remediatees to preserve as much autonomy as possible might facilitate not only individual remediatee engagement in the process, but also that of universities and university Continuing Professional Development(CPD) offices.

Our research results lead to a number of recommendations, some of which I was able to share with the attendees at the national symposium, attendees who have the authority to make changes. Others have been developed when considering the PhD project as a whole. These recommendations include both conceptual and practical changes. 
Recommendations for conceptual changes include:

- Rather than thinking about remediation as education, conceptualizing it as supported practice change, and focusing on facilitating the individual's motivation and ability to change by providing personal, social and structural supports.

- Changing the questions we ask when developing remediation programs. For example, instead of asking ourselves what are the characteristics of successfully remediated physicians, we might reframe the question to ask how we determine whether remediation goals are feasible for a particular physician in a particular context.

- Focusing on how we can ensure that programmatic structures facilitate long-term change rather than short-term achievement of learning objectives.

- Conceptualizing the education/regulation duality of remediation as a polarity and focusing on remaining in the upper/positive quadrants of the polarity map i.e. on maximizing the benefits of each conceptualization and minimizing the disadvantages.

Recommendations for practical changes include:

- Ensuring that what individual organizations learn about remediation be published in scholarly journals, and not be available only in the grey literature, where it can disappear/be lost over time.

- Engaging remediatees in the construction of remediation plans that provide them with as much autonomy as possible.

- Ensuring that the remediator, and the person assessing the success of the remediation are two different individuals.

- Developing and supporting a remediator community of practice. This includes advocating for their work to be understood as the important contribution to the community that it is, and ensuring that it is considered in tenure decisions.

- Providing faculty development for remediators that addresses the concerns identified by our remediator participants. These concerns include: lack of information provided by those mandating the remediation, uncertainty regarding the extent to which they are allowed to modify the process as they see fit in a particular circumstance, dealing with boundary issues, unique logistical challenges such as remediating in a small community, and dealing with power and gender imbalances. In addition, our research suggests that discussing how to identify the positions of educator, judge and public defender, and move gracefully between them as needed would facilitate the remediator role. 
We also found that much of what is available regarding remediation in practice, particularly data on programs and outcomes, is never published, or else appears in the grey literature, only to subsequently disappear again. Much of what individual remediation programs learn is either kept internally or shared at conferences and meetings of regulators or program developers i.e. remains within a small community. Our results suggest that it is time to make this data more widely available. Not only might this encourage more researchers to study this area, wider dissemination of remediation practices and outcomes would counter the perception that remediation is an extremely rare, and therefore shameful, event. At the aforementioned meeting, I made a plea for wider dissemination of program data. I believe I can state that the entire presentation was very well received, as evidenced by the session evaluations. One regulatory authority representative stated afterwards that they had never stopped to think about what remediation actually is, and that this would change their conception of it.

Our research results could thus be used to refine existing remediation processes and programs as well as for the development of new programs. They also have implications for how remediation is presented to individuals whose practice gaps require addressing, as well as for how it is presented to potential participating organizations. Acknowledging both the educational and regulatory aspects of remediation, and developing processes that focus on maximizing the upsides of both, while minimizing the challenges of each might help engage remediatees. For example, one way to diminish remediatee resistance might be to encourage them to focus on the educational aspects by allowing them to retain as much autonomy as possible in individual program design and the development of learning objectives. Taking into consideration the 'social determinants of competence' in each case might both improve long-term results and convince remediatees that this process is indeed about helping improve performance, and not about 'shame and blame'. It would also suggest that we need to look at the role of the health care system and of the workplace in facilitating and in some cases, impeding, competence.

Our results suggest that remediation programs should be aware that remediation is both educational and regulatory, and that this dual nature needs to be acknowledged and kept in mind when developing processes. Programs might focus on maximizing the positive aspects of both remediation as education and remediation as regulation, while simultaneously focusing on minimizing the downsides of each. For example, in regards to remediation as education, they might emphasize not only closing the knowledge, skills or attitude gap, but also developing skills so as to avoid future need for remediation. Integrating remediation into the CPD education system so as normalize it and decrease the stigma, and making it constructive, not punitive, might improve engagement in all quarters. At the same time, remediation program developers might want to ensure that the focus on education does not de-emphasize the seriousness of the situation the individual finds themselves in, nor the fact that the ultimate stakeholder, the individual whose well-being comes first, is the patient/public, not the individual physician/the profession. Hence the simultaneous focus on remediation as regulation. It should be made perfectly clear that practice change is not optional, and that the individual has failed the important professional responsibility of self-regulating to ensure that they provide competent, up-to-date care. However, 'shaming and blaming' and treating the individual 
as 'a bit of a tainted product', as one of our participants put it, serves neither the individual being remediated nor the broader public. Remediation programs focusing on maximizing the upsides and minimizing the downsides of both education and regulation might also help maximize positive outcomes, although proof of that would require further research.

Our finding that stakeholders carry ambivalent attitudes towards remediation and remediatees suggests that the profession needs to find ways to address these attitudes. However, since culture change is notoriously difficult, it may be easier to proceed in the opposite direction i.e. rather than wait for culture change in order to develop remediation programs, we might seek instead to normalize remediation by integrating it into the CPD curriculum. This might help address one of the biggest challenges for remediation program directors (according to those through whom we went to access participants for our remediator study), that of recruiting preceptors. There are likely several reasons for this, including the general discomfort with remediation identified in our work, as well as lack of familiarity with remediation and thus hesitance to engage. Our findings, however, show that working with remediatees can be profoundly gratifying; sharing the deep satisfaction that can come from doing this work might help with recruitment. On the other hand, our remediator participants also shared challenges, which, if not addressed, might discourage potential remediators. Our results will then also be of interest to those who recruit remediators and those who provide faculty development for them.

In addition to those directly involved in the remediation of practicing physicians, our results might also be of interest to undergraduate and postgraduate academic program directors who may wish to consider whether some of the attitudes that we found at the practice level and their related challenges are also present to some extent at earlier points in the continuum. At least one of our participants suggested that such attitudes are evident across the continuum. Several of our stakeholder participants mentioned their concern that if professional organizations don't deal with dyscompetent colleagues, external forces such as governments, will do it for them. Our results are thus very relevant to such organizations, and suggest the need for more engagement with remediation than they have hitherto been willing to provide. Our results may also be indirectly beneficial to patients, by helping have that the profession deals with dyscompetent colleagues. Removing such physicians from practice or limiting privileges is, of course, another, perhaps faster, way to address incompetence, but that route is frequently associated with patients losing access to health care or to a provider they have come to know and trust. Patients thus have a stake in remediation.

Our results are innovative in that they suggest previously undiscussed reasons behind barriers to the development of more widespread remediation programs. We have uncovered hitherto undiscussed obstacles to service development, in this case, to the development of services to support, coach and guide physicians whose practice in some way fails to meet the standard. The literature and presentations at meetings and conferences tended to focus on logistical barriers such as funding, clinical resources (e.g. competition for patients with postgraduate learners, particularly for surgical and other procedures), and time. It will be difficult to overcome these logistical barriers without 
addressing the ambivalence towards both the process of remediation and towards those individuals who require it.

I have been sharing these results with individuals and groups outside academia who are directly involved in the remediation of practicing physicians. They have suggested further avenues for research, such as directly talking with remediatees, which I hope to pursue. The regulators have not only expressed an interest in the potential results but also given suggestions as to some of the questions they would like answered.

I would hope that by adopting some of these ideas and keeping in mind our findings as they refine their processes, regulatory authorities and universities will find it easier to recruit and support remediators, and to engage remediatees in practice improvement. These ideas are not necessarily more costly to implement than current practices; they require a shifting of focus more than an injection of new resources. Ultimately, I hope that by starting a conversation about how we as a profession view remediation and remediatees, that some of the stigma can be reduced so that remediation occurs earlier with the result that practice gaps are addressed before they become too broad or deep. 

Acknowledgements 

I would like to first thank my supervisors, each of whom brought unique perspectives and expertise and provided different insights and guidance along the way. Together, they constituted what I refer to as my "dream team". They provided encouragement, direction, and gentle persuasion when needed. They made pursuing a PhD truly one of the best experiences of my life.

I would also like to thank the Centre for Health Education Scholarship (CHES) at UBC, who provided me with an academic 'home' and prevented me from feeling too isolated here on the island.

While I am not much of a social media aficionada and rarely posted, the Facebook group of older PhD students, the "PhD OWLs" (일 Wise Learners) provided me with perspective and resources, and helped me realize even more how fortunate I have been in the whole PhD process.

I am grateful to my participants who generously gave of their time and perspectives.

Finally, thank you to my husband of 43 years, Randy, whose unwavering support throughout the process and uncomplaining acceptance of "but I have to work on the PhD now" was much appreciated. 

Curriculum Vitae 

Gisèle Bourgeois-Law was born on November 2, 1956, in Winnipeg, Manitoba. She received her MD from the University of Manitoba in 1979, followed by her certification from the College of Family Physicians of Canada in 1981. She soon noticed that her patients were overwhelmingly female, and since she very much enjoyed obstetrics, decided to switch specialties and received her certification as an obstetrician-gynecologist through the Royal College of Physicians and Surgeons of Canada in 1986. The combination of two children, a busy practice, and a system that at the time required her to attend at two different hospitals (she once delivered 22 babies in 24 hours between the 2 hospitals) led to what can be considered either an epiphany or burn-out, and she eventually left her hospital practice. The next few years were spent in an office practice focused on patients with chronic pelvic pain, menopause challenges, and sexual dysfunction. She obtained an Applied Counselling Certificate, which served her well not only in her practice, but also led to her being asked to direct the Clinician Assessment and Professional Enhancement (CAPE) Program, a program that assesses and arranges remediation for physicians referred from the regulatory authority for everything from clinical competence issues to crossing sexual boundaries.

Over the course of her career, in addition to her practice and her 10 years as CAPE Director, Gisèle has served in Manitoba as Associate Dean Continuing Medical Education, Site Coordinator Physician Assistant Students, and Acting Head of the Department of Medical Education. She obtained both her Masters of Education and a Certificate in University and College Administration through the University of Manitoba.

In 2008, Gisèle moved to British Columbia to take up the position of Associate Dean Professional Development at UBC. During her time there, she also served as the Acting Director of the Evaluation Studies Unit during the director's sabbatical. Pursuing a PhD had been a goal since the Masters, but Gisèle reluctantly came to the realization that while many people manage a PhD and full-time work, she was probably not one of those people. She therefore resigned from her position and, while pursuing her $\mathrm{PhD}$, has been enjoying serving as the Liaison between the Centre for Health Education Scholarship at UBC and the Island Medical Program in Victoria, as well as working with medical students in various capacities.

Gisèle is grateful to have had the opportunity to do a PhD at this stage in her life, when she could enjoy the process and not worry about finding an academic position in precarious times. She hopes to continue doing research in the area of remediation. 


\section{SHE dissertations series}

The SHE Dissertation Series publishes dissertations of PhD candidates from the School of Health Professions Education (SHE) who defended their PhD theses at Maastricht University. The most recent ones are listed below. For more information go to: https://she. mumc.maastrichtuniversity.nl

Giuliani, M. (19-05-2020) A Critical Review of Global Curriculum Development, Content and Implementation in Oncology

Schreurs, S. (20-03-2020) Selection for medical school; the quest for validity

Schumacher, D. (19-03-2020) Resident Sensitive Quality Measures: Defining the Future of Patient-Focused Assessment

Sehlbach, C. (21-02-2020) To be continued.... Supporting physicians' lifelong learning

Kikukawa, M. (17-12-2019) The situated nature of validity: Exploring the cultural dependency of evaluating clinical teachers in Japan

Kelly, M. (10-12-2019) Body of knowledge. An interpretive inquiry into touch in medical education

Klein, D. (06.11.2019) The performance of medical record review as an instrument for measuring and improving patient safety

Bollen, J. (01.11.2019) Organ donation after euthanasia: medical, legal and ethical considerations

Wagner-Menghin, M. (25-09-2019) Self-regulated learning of history-taking: looking for predictive cues

Wilby, K. (02-07-2019) When numbers become words: Assessors'processing of performance data within OSCEs

Szulewski, A. (20-06-2019) Through the eyes of the physician: Expertise development in resuscitation medicine

McGill, D. (29-05-2019) Supervisor competence as an assessor of medical trainees; Evaluating the validity and quality of supervisor assessments

Van Rossum, T. (28-02-2019) Walking the tightrope of training and clinical service; The implementation of time variable medical training 



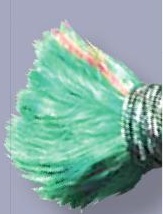

